\title{
A SEARCH FOR EXTENDED ULTRAVIOLET DISK (XUV-DISK) GALAXIES IN THE LOCAL UNIVERSE
}

\author{
David A. Thilker, ${ }^{1}$ Luciana Bianchi, ${ }^{1}$ Gerhardt Meurer, ${ }^{1}$ Armando Gil de Paz, ${ }^{2}$ Samuel Boissier, ${ }^{3}$ \\ Barry F. Madore, ${ }^{4}$ Alessandro Boselli, ${ }^{3}$ Annette M. N. Ferguson, ${ }^{5}$ Juan Carlos Muńoz-Mateos, ${ }^{2}$ \\ Greg J. Madsen, ${ }^{6}$ Salman Hameed, ${ }^{7}$ Roderik A. Overzier, ${ }^{1}$ Karl Forster, ${ }^{8}$ Peter G. Friedman, ${ }^{8}$ \\ D. Christopher Martin, ${ }^{8}$ Patrick Morrissey, ${ }^{8}$ Susan G. Neff, ${ }^{9}$ David Schiminovich, ${ }^{10}$ \\ Mark Seibert, ${ }^{8}$ Todd Small, ${ }^{8}$ Ted K. Wyder, ${ }^{8}$ José Donas, ${ }^{3}$ Timothy M. Heckman, ${ }^{11}$ \\ Young-Wook Lee, ${ }^{12}$ Bruno Milliard, ${ }^{3}$ R. Michael Rich, ${ }^{13}$ Alex S. Szalay, ${ }^{11}$ \\ BarRy Y. Welsh, ${ }^{14}$ and SuKYOUng K. YI ${ }^{12}$ \\ Received 2007 April 30; accepted 2007 September 11
}

\begin{abstract}
We have initiated a search for extended ultraviolet disk (XUV-disk) galaxies in the local universe. Here we compare GALEX UV and visible-NIR images of 189 nearby $(D<40 \mathrm{Mpc}) \mathrm{S} 0-\mathrm{Sm}$ galaxies included in the GALEX Atlas of Nearby Galaxies and present the first catalog of XUV-disk galaxies. We find that XUV-disk galaxies are surprisingly common but have varied relative (UV/optical) extent and morphology. Type 1 objects ( $220 \%$ incidence) have structured, UV-bright/optically faint emission features in the outer disk, beyond the traditional star formation threshold. Type 2 XUV-disk galaxies ( $\sim 10 \%$ incidence) exhibit an exceptionally large, UV-bright/optically low surface brightness (LSB) zone having blue $\mathrm{UV}-K_{s}$ outside the effective extent of the inner, older stellar population, but not reaching extreme galactocentric distance. If the activity occurring in XUV-disks is episodic, a higher fraction of present-day spirals could be influenced by such outer disk star formation. Type 1 disks are associated with spirals of all types, whereas Type 2 XUV-disks are predominantly found in late-type spirals. Type 2 XUV-disks are forming stars quickly enough to double their (currently low) stellar mass in the next Gyr (assuming a constant star formation rate). XUV-disk galaxies of both types are systematically more gas-rich than the general galaxy population. Minor external perturbation may stimulate XUV-disk incidence, at least for Type 1 objects. XUV-disks are the most actively evolving galaxies growing via inside-out disk formation in the current epoch, and may constitute a segment of the galaxy population experiencing significant, continued gas accretion from the intergalactic medium or neighboring objects.
\end{abstract}

Subject headings: galaxies: evolution — ultraviolet: galaxies

Online material: machine-readable table, extended figure set

\section{INTRODUCTION}

Imprinted within the disk component of any spiral galaxy is a wealth of information regarding its formation, and clues concerning its continued evolution. For instance, radial variations in the average surface brightness of the stellar disk are not purely exponential with a sharp outer truncation, as once thought (e.g., van der

\footnotetext{
1 Center for Astrophysical Sciences, The Johns Hopkins University, 3400 North Charles Street, Baltimore, MD 21218; dthilker@pha.jhu.edu.

2 Departamento de Astrofísica, Universidad Complutense de Madrid, Madrid 28040, Spain.

${ }^{3}$ Laboratoire d'Astrophysique de Marseille, BP 8, Traverse du Siphon, 13376 Marseille Cedex 12, France.

4 Observatories of the Carnegie Institution of Washington, 813 Santa Barbara Street, Pasadena, CA 91101.

5 Institute for Astronomy, University of Edinburgh, Royal Observatory Edinburgh, Edinburgh, UK.

6 School of Physics A29, University of Sydney, NSW 2006, Australia.

7 Five College Astronomy Department, Hampshire College, Amherst, MA 01003

8 California Institute of Technology, MC 405-47, 1200 East California Boulevard, Pasadena, CA 91125

9 Laboratory for Astronomy and Solar Physics, NASA Goddard Space Flight Center, Greenbelt, MD 20771.

10 Department of Astronomy, Columbia University, New York, NY 10027.

11 Department of Physics and Astronomy, The Johns Hopkins University, Homewood Campus, Baltimore, MD 21218.

12 Center for Space Astrophysics, Yonsei University, Seoul 120-749, Korea.

13 Department of Physics and Astronomy, University of California, Los Angeles, CA 90095

14 Space Sciences Laboratory, University of California at Berkeley, 601 Campbell Hall, Berkeley, CA 94720.
}

Kruit \& Searle 1981; Florido et al. 2001), but rather are best represented as a complex broken exponential (Pohlen \& Trujillo 2006; Erwin et al. 2005; Pohlen et al. 2002), sometimes even flattening in the outer disk. The color of the disk is also a function of galactocentric distance (e.g., de Jong 1996; MacArthur 2006; MacArthur et al. 2004; Muńoz-Mateos et al. 2007). Such complex surface brightness and color profiles trace changes in the disk stellar population, and reflect the assembly history of the galaxy, modulated by the combined effects of radial motion (of secular or external origin) and extinction. The chemical evolution of a galaxy is also driven by the star formation history (Boissier \& Prantzos 2000; Chiappini et al. 2003). Abundance gradients typically reveal a comparatively enriched inner disk (Zaritsky et al. 1994; Henry \& Worthey 1999), although barred spirals are less steep in general. The overall picture which emerges is that the outer disk is often the locale of most significant recent change, although it remains considerably less evolved, as expected for inside-out disk formation (White \& Frenk 1991; Mo et al. 1998; Boissier \& Prantzos 1999; Brook et al. 2006; Muńoz-Mateos et al. 2007). Quantifying the recent star formation rate (SFR) across the entire disk in a large galaxy sample will provide an anchor for the interpretation of enrichment and disk-building processes.

In a broader sense, understanding the typical star formation activity of outer disks is crucial because it has a bearing on diverse topics, including absorption-line diagnostics of metal abundance in the circumgalactic medium, efforts to determine how (and at what rate) gas accretes onto seemingly evolved galaxies, and how star formation is regulated (or inhibited) in low-density regions perhaps similar to protogalactic environments in the early universe. 
It is known from deep $\mathrm{H} \alpha$ imaging that some galaxies possess very extended star-forming disks (e.g., Ferguson et al. 1998b). However, the incidence and underlying cause of this behavior are still unknown. The new ultraviolet imaging surveys from NASA's GALEX (Galaxy Evolution Explorer) mission greatly facilitate the study of low-intensity, outer disk star formation in a statistically significant number of galaxies (Gil de Paz et al. 2007a). GALEX imaging benefits from a very low sky background, high sensitivity, and a large field of view ( $1.25^{\circ}$ diameter), enabling efficient detection of otherwise elusive activity. By observing in the UV, $G A L E X$ probes a population of OB stars (rather than only the higher temperature $\mathrm{O}$ stars predominantly ionizing $\mathrm{H}$ II regions traced by $\mathrm{H} \alpha$ emission), and thus can catch galaxy evolution processes occurring at very low SFR surface densities $\left(\Sigma_{\mathrm{SFR}}\right)$.

Thilker et al. (2005a) and Gil de Paz et al. (2005) discovered extended UV (XUV) emission in the extreme outer disk environment of M83 and NGC 4625. Both these studies established that the amount and spatial extent of star formation in the outskirts of a galaxy can be underestimated by looking for $\mathrm{H}$ II regions alone (as traced by $\mathrm{H} \alpha$ emission). However, a multitude of unanswered questions follow from the XUV-disk discovery. For instance, are XUV-disks rare or relatively common (e.g., Zaritsky \& Christlein 2007)? Are certain galaxy types more likely to host spatially extended star formation? What is the characteristic morphology of XUV emission? Is the SF episodic, or continuous? M83 and NGC 4625 are rather dissimilar in their extended UV morphology. Does that suggest that the outer disk has a different origin or star formation history (SFH), or have we simply caught these particular XUV-disks at different stages in their evolutionary progression? Outer disk star formation does have an impact on the course of chemical evolution. Gil de Paz et al. (2007b) show that the metallicity of XUV-disks (M83, NGC 4625) is very low, although not primordial.

Much attention has been directed toward the evidence in favor of a star formation threshold in low-density environments (e.g., Kennicutt 1989; Martin \& Kennicutt 2001). Indeed, many galaxies appear to show a sharp decline in the radially averaged SFR per unit area derived from sensitive $\mathrm{H} \alpha$ observations, occurring at the radius where the disk is predicted to become dynamically stable. The discovery of XUV-disks raised questions about this interpretation. Boissier et al. (2007) demonstrate that at least some truncations in $\mathrm{H} \alpha$ may be an artifact of small-number statistics. Specifically, the number of $\mathrm{H}$ II regions expected at any given moment in the discrete radial bins used to compute $\mathrm{H} \alpha$ surface brightness profiles becomes vanishingly small for the observed, UV-derived $\Sigma_{\text {SFR }}$ at such locations.

This paper reports on a campaign to better understand the properties of XUV-disk galaxies and their relevance in a cosmological context. Here we determine the incidence of XUV emission in a large sample of nearby disk galaxies. Necessarily, we suggest a classification scheme for defining the presence of XUV-disks. The large number ( $\sim 200)$ of galaxies considered in our study helps to better constrain the conditions which allow or encourage an XUV-disk to form. Our analysis includes a look at the importance of galactic environment (isolation vs. group membership) and the presence of dynamically perturbing companions. The present paper also considers the relation of spatially extended star formation (traced in the UV) with optical surface brightness profiles of various types (Pohlen \& Trujillo 2006; Erwin et al. 2005). In particular, we comment on the possible connection of "antitruncated" optical disks and low surface brightness (LSB) galaxies with XUV-disks.

Section 2 describes our galaxy sample along with the GALEX and ground-based visible-NIR observations we used for this study.
Section 3 outlines our data analysis and XUV-disk galaxy classification scheme. We also examine global galaxy parameters and environmental properties of the XUV-disk class in comparison to the entire sample. Section 4 presents an extensive discussion and our main conclusions are listed in $\S 5$. In Appendix A, we provide a description and multiwavelength imaging for each galaxy in our XUV-disk catalog. Appendix B presents a short summary of the inside-out disk formation models used to compare with observed galaxy characteristics.

\section{SAMPLE SELECTION AND OBSERVATIONS}

\subsection{The Galaxy Sample}

Our survey sample (Table 1) comprises 189 disk galaxies $(-0.5 \leq T \leq 9.5$, consequently S0-Sm) within $40 \mathrm{Mpc}$ included in the $\bar{G} A L E X$ Atlas of Nearby Galaxies (Gil de Paz et al. 2007a) and having $i \leq 80^{\circ}, D_{25}>90^{\prime \prime}$, in addition to modest Galactic extinction, $A_{V} \leq 0.5$. By selecting only objects from the Atlas, we ensure that all galaxies were observed with homogeneous (deep) sensitivity. M31 and M33 have been excluded, as their large angular size significantly complicates our analysis. The upper distance limit ensures that spatial resolution of our GALEX observations is $\sim 1 \mathrm{kpc}$ or less.

Galaxy distances were assigned following Gil de Paz et al. (2007a), who established a ranking of reliability for various possible indicators (e.g., Cepheids, tip of the red giant branch, etc.) with use of the Hubble constant $\left(H_{0}=70 \mathrm{~km} \mathrm{~s}^{-1} \mathrm{Mpc}^{-1}\right)$ adopted only as a fallback method.

LEDA was employed to extract ancillary quantities, such as rotation velocities, mean surface brightness, $\mathrm{H}$ I mass, and $M_{\mathrm{H}_{\mathrm{I}}} / L_{B}$. LEDA contains $M_{\mathrm{H} \text {, }}$ for almost all ( $>90 \%$ ) of our targets, even if some of these (single-dish) H I measurements could be underestimates in the case of angularly large galaxies. Furthermore, Gil de Paz et al. (2007a) and Dale et al. (2007) published observed panchromatic spectral energy distributions (SEDs) for the GALEX Atlas sample and the SINGS sample (Kennicutt et al. 2003), respectively. These data were used to compute galaxy-averaged quantities such as the extinction-free SFR (from the union of UV and TIR luminosity), extinction-free specific SFR (sSFR = SFR/ $M_{*}=$ star formation rate per unit of stellar mass), and the ratio of infrared to ultraviolet luminosity (TIR/UV). SFR surface density $\left(\Sigma_{\mathrm{SFR}}\right)$ was estimated very crudely using $D_{25}$ as the fiducial disk size. Note that the SFR used for computing $\Sigma_{\mathrm{SFR}}$ sometimes includes SF outside of the $D_{25}$ radius, as we only have galaxyintegrated values of IR luminosity to use in the calculation. For consistency, the UV measurements used for $\Sigma_{\text {SFR }}$ are also galaxyintegrated. The dynamical and photometric quantities considered in our study are included in Table 1.

In order to quantify the environment of each galaxy, we referenced the hierarchical group assignments of the Giuricin et al. (2000) Nearby Optical Galaxy (NOG) sample. This study of a magnitude-limited $(B \leq 14)$ sample of nearby galaxies $\left(c z_{\mathrm{LG}} \leq\right.$ $6000 \mathrm{~km} \mathrm{~s}^{-1}$ ) showed that $40 \%$ of the local galaxy population is ungrouped, and the remaining $60 \%$ is distributed within galaxy pairs $(15 \%)$ and more populous groups $(45 \%)$. In Table 1 , for each of our 189 galaxies we list the common name of the associated NOG group (when available) or else the brightest galaxy in the NOG group, in addition to the number of galaxies in each group. Being part of a group does not guarantee that an object is currently (or recently) perturbed via galaxy interaction. Therefore, we also computed the tidal perturbation parameter for all sample galaxies (see $\S 4$ for details). Finally, note that the Giuricin et al. study is expected to miss faint companions of our sample galaxies (especially at the more distant end of our sample), and 
TABLE 1

40 Mpc Disk Galaxy Sample

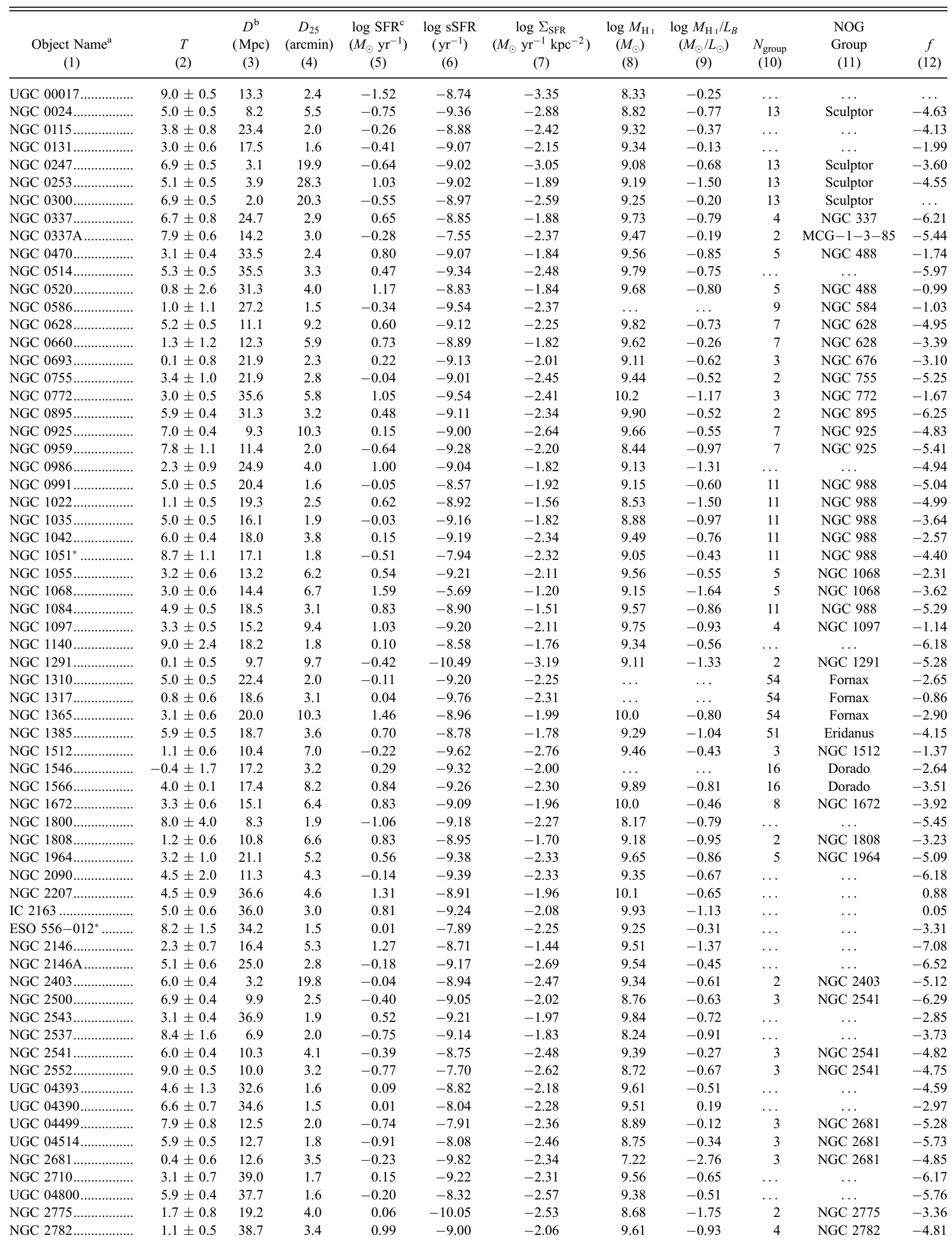


TABLE 1-Continued

\begin{tabular}{|c|c|c|c|c|c|c|c|c|c|c|c|}
\hline $\begin{array}{l}\text { Object Name }{ }^{\mathrm{a}} \\
\text { (1) }\end{array}$ & $\begin{array}{c}T \\
(2)\end{array}$ & $\begin{array}{c}D^{\mathrm{b}} \\
(\mathrm{Mpc}) \\
(3)\end{array}$ & $\begin{array}{c}D_{25} \\
\text { (arcmin) } \\
(4)\end{array}$ & $\begin{array}{c}\log \mathrm{SFR}^{\mathrm{c}} \\
\left(M_{\odot} \mathrm{yr}^{-1}\right) \\
(5)\end{array}$ & $\begin{array}{c}\log \mathrm{sSFR} \\
\left(\mathrm{yr}^{-1}\right) \\
(6)\end{array}$ & $\begin{array}{c}\log \sum_{\mathrm{SFR}} \\
\left(M_{\odot} \mathrm{yr}^{-1} \mathrm{kpc}^{-2}\right) \\
(7)\end{array}$ & $\begin{array}{c}\log M_{\mathrm{H}} \\
\left(M_{\odot}\right) \\
(8)\end{array}$ & $\begin{array}{c}\log M_{\mathrm{H}_{\mathrm{I}}} / L_{B} \\
\left(M_{\odot} / L_{\odot}\right) \\
(9)\end{array}$ & $\begin{array}{l}N_{\text {group }} \\
\text { (10) }\end{array}$ & $\begin{array}{c}\text { NOG } \\
\text { Group } \\
(11)\end{array}$ & $\begin{array}{c}f \\
(12)\end{array}$ \\
\hline 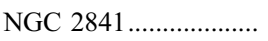 & $3.0 \pm 0.7$ & 14.1 & 8.1 & 0.28 & -9.96 & -2.66 & 9.75 & -0.93 & 2 & NGC 2841 & -4.62 \\
\hline NGC 2976...................... & $5.3 \pm 0.8$ & 3.6 & 6.0 & -0.74 & -9.20 & -2.23 & 8.09 & -1.18 & 9 & M81 & -3.11 \\
\hline NGC 3023...................... & $5.4 \pm 0.8$ & 26.4 & 1.9 & 0.27 & -8.75 & -1.95 & 9.53 & -0.49 & 7 & NGC 2967 & 0.21 \\
\hline 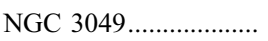 & $2.5 \pm 0.7$ & 21.6 & 2.1 & 0.00 & -9.05 & -2.14 & 9.03 & -0.63 & & & -5.90 \\
\hline NGC $3031^{*} \ldots \ldots \ldots \ldots \ldots$ & $2.4 \pm 0.6$ & 3.6 & 21.7 & 0.03 & -9.92 & -2.59 & 9.46 & -1.05 & 9 & M81 & -2.05 \\
\hline ESO $435-016^{*} \ldots \ldots \ldots . .$. & $3.4 \pm 2.3$ & 11.7 & 1.8 & -0.38 & -8.81 & -1.85 & 8.80 & -0.38 & 9 & NGC 3175 & -4.93 \\
\hline 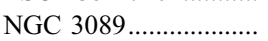 & $3.2 \pm 0.9$ & 36.4 & 1.8 & 0.66 & -9.10 & -1.79 & 8.82 & -1.49 & 3 & IC 2537 & -4.74 \\
\hline ESO $499-037^{*} \ldots \ldots \ldots . .$. & $6.2 \pm 1.5$ & 11.5 & 3.3 & -0.43 & -7.84 & -2.40 & 9.00 & -0.37 & 9 & NGC 3175 & -4.54 \\
\hline 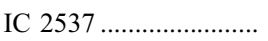 & $5.0 \pm 0.4$ & 37.6 & 2.5 & 0.63 & -9.13 & -2.13 & 9.75 & -0.74 & 3 & IC 2537 & -5.21 \\
\hline 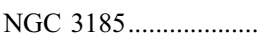 & $1.1 \pm 1.0$ & 17.4 & 2.0 & -0.40 & -9.59 & -2.29 & 8.42 & -1.23 & 8 & NGC 3190 & -2.57 \\
\hline 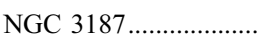 & $5.0 \pm 0.8$ & 17.4 & 2.3 & -0.04 & -8.55 & -2.08 & 8.85 & -0.68 & .. & $\ldots$ & -1.12 \\
\hline 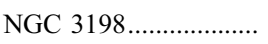 & $5.2 \pm 0.6$ & 16.6 & 7.6 & 0.41 & -9.29 & -2.62 & 9.99 & -0.66 & 5 & NGC 3198 & -5.00 \\
\hline ESO $317-023^{*} \ldots \ldots \ldots$ & $1.1 \pm 0.6$ & 37.9 & 1.9 & 0.99 & -8.33 & -1.56 & $\ldots$ & $\ldots$ & .. & $\ldots$ & -4.18 \\
\hline 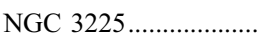 & $5.9 \pm 0.6$ & 33.7 & 1.6 & 0.06 & -8.90 & -2.23 & 9.57 & -0.52 & 2 & NGC 3225 & -4.91 \\
\hline 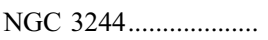 & $5.6 \pm 0.8$ & 36.5 & 2.0 & 0.53 & -9.11 & -2.04 & 9.53 & -0.88 & . & $\ldots$ & -3.10 \\
\hline 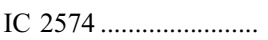 & $8.9 \pm 0.4$ & 4.0 & 11.8 & -0.76 & -8.05 & -2.94 & 9.12 & -0.26 & 9 & M81 & -3.25 \\
\hline UGC $05675 \ldots \ldots \ldots \ldots \ldots$ & $8.6 \pm 1.1$ & 16.8 & 1.6 & -1.48 & -8.90 & -3.16 & 8.42 & -6.22 & . & & -3.30 \\
\hline NGC 3277 .................... & $1.8 \pm 0.8$ & 21.8 & 1.9 & -0.34 & -9.82 & -2.42 & 8.66 & -1.32 & 5 & NGC 3254 & -4.89 \\
\hline 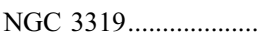 & $5.9 \pm 0.4$ & 14.6 & 4.9 & 0.22 & -8.45 & -2.32 & 9.49 & -0.56 & 5 & NGC 3198 & -5.42 \\
\hline 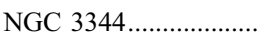 & $4.0 \pm 0.3$ & 6.9 & 6.7 & -0.14 & -9.20 & -2.29 & 9.14 & -0.60 & 3 & NGC 3344 & -6.57 \\
\hline NGC $3351^{*} \ldots \ldots \ldots \ldots \ldots$ & $3.0 \pm 0.6$ & 11.8 & 7.1 & 0.36 & -9.48 & -2.30 & 9.15 & -1.12 & 24 & Leo I & -3.01 \\
\hline 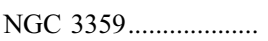 & $5.2 \pm 0.5$ & 18.0 & 5.7 & 0.45 & -8.98 & -2.39 & 9.98 & -0.46 & $\ldots$ & $\ldots$ & -5.51 \\
\hline NGC $3368^{*} \ldots \ldots \ldots \ldots \ldots$ & $1.8 \pm 0.5$ & 13.5 & 7.4 & 0.25 & -9.85 & -2.58 & 9.45 & -1.08 & 24 & Leo I & -3.31 \\
\hline NGC 3377A ..................... & $8.8 \pm 0.5$ & 9.1 & 1.6 & -1.62 & -8.49 & -2.74 & 8.01 & -0.41 & $\ldots$ & $\ldots$ & -1.57 \\
\hline NGC 3486....................... & $5.2 \pm 0.8$ & 11.6 & 6.1 & 0.15 & -9.15 & -2.37 & 9.46 & -0.58 & 2 & NGC 3486 & -4.39 \\
\hline NGC 3521....................... & $4.0 \pm 0.3$ & 9.0 & 9.6 & 0.52 & -9.44 & -2.18 & 9.54 & -0.92 & $\ldots$ & $\ldots$ & -6.06 \\
\hline 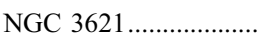 & $6.9 \pm 0.4$ & 8.3 & 10.4 & 0.47 & -9.09 & -2.23 & 9.86 & -0.53 & $\ldots$ & $\ldots$ & $\ldots$ \\
\hline 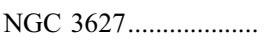 & $3.0 \pm 0.4$ & 9.1 & 8.9 & 0.63 & -9.30 & -2.00 & 8.88 & -1.55 & 24 & Leo I & -2.12 \\
\hline 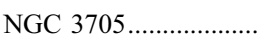 & $2.4 \pm 0.6$ & 15.3 & 4.3 & 0.00 & -9.56 & -2.46 & 9.32 & -0.83 & 24 & Leo I & -5.39 \\
\hline 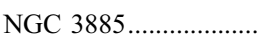 & $0.2 \pm 1.0$ & 23.1 & 2.4 & 0.49 & -9.25 & -1.82 & 9.27 & -0.70 & 3 & NGC 3885 & -4.46 \\
\hline ESO $440-011^{*} \ldots \ldots \ldots . .$. & $6.9 \pm 0.5$ & 23.1 & 2.6 & 0.04 & -7.65 & -2.34 & 9.37 & -0.58 & 3 & NGC 3936 & -3.64 \\
\hline NGC 3938...................... & $5.1 \pm 0.5$ & 12.2 & 4.4 & 0.28 & -9.13 & -1.99 & 9.34 & -0.71 & 25 & NGC 4151 & -4.94 \\
\hline NGC 4030........................ & $4.0 \pm 0.2$ & 21.0 & 3.6 & 0.92 & -9.16 & -1.67 & 9.62 & -0.88 & 3 & NGC 4030 & -4.09 \\
\hline NGC 4038........................ & $8.9 \pm 0.5$ & 22.2 & 4.4 & 0.54 & -7.11 & -2.26 & 9.55 & -1.21 & 17 & NGC 4038 & 0.78 \\
\hline 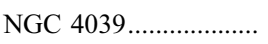 & $8.9 \pm 0.2$ & 22.2 & 4.0 & -0.11 & -7.77 & -2.83 & $\ldots$ & $\ldots$ & 17 & NGC 4038 & 0.91 \\
\hline 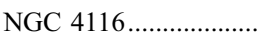 & $7.5 \pm 1.1$ & 17.0 & 2.9 & -0.01 & -8.73 & -2.22 & 9.40 & -0.48 & 4 & NGC 4179 & -2.72 \\
\hline NGC 4136..................... & $5.2 \pm 0.7$ & 10.9 & 2.6 & -0.36 & -9.08 & -2.08 & 8.98 & -0.56 & 27 & Coma I & -4.45 \\
\hline 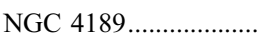 & $6.0 \pm 0.9$ & 32.0 & 2.8 & 0.57 & -9.24 & -2.16 & 9.37 & -0.99 & 9 & Virgo M & -3.38 \\
\hline NGC $4192^{*} \ldots \ldots \ldots \ldots \ldots$ & $2.5 \pm 0.8$ & 17.0 & 9.5 & 0.36 & -9.71 & -2.88 & 9.68 & -0.99 & 159 & Virgo A & $\ldots$ \\
\hline 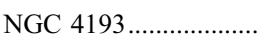 & $4.1 \pm 1.8$ & 32.0 & 2.0 & 0.30 & -9.34 & -2.14 & 9.15 & -1.04 & 9 & Virgo M & -3.14 \\
\hline NGC 4236..................... & $7.9 \pm 0.5$ & 4.5 & 20.4 & -0.55 & -8.61 & -3.29 & 9.22 & -0.66 & 2 & NGC 4236 & -5.51 \\
\hline 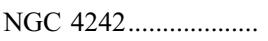 & $8.0 \pm 0.4$ & 10.3 & 4.2 & -0.52 & -7.51 & -2.62 & 8.96 & -0.78 & 21 & NGC 4258 & -4.09 \\
\hline NGC 4248....................... & $2.9 \pm 2.7$ & 7.2 & 2.3 & -1.07 & -8.91 & -2.31 & 7.77 & -1.11 & 21 & NGC 4258 & -1.58 \\
\hline NGC 4254*.................... & $5.2 \pm 0.6$ & 17.0 & 5.1 & 1.02 & -9.03 & -1.67 & 9.68 & -0.92 & 159 & Virgo A & -5.52 \\
\hline NGC 4258* ..................... & $4.0 \pm 0.2$ & 7.2 & 17.4 & 0.26 & -9.63 & -2.76 & 9.64 & -0.90 & 21 & NGC 4258 & -2.20 \\
\hline 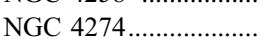 & $1.7 \pm 0.6$ & 16.0 & 5.0 & 0.04 & -9.92 & -2.59 & 8.67 & -1.61 & 27 & Coma I & -2.61 \\
\hline NGC 4298....................... & $5.2 \pm 0.5$ & 17.0 & 2.7 & 0.41 & -9.03 & -1.75 & 8.95 & -1.11 & 159 & Virgo A & -0.72 \\
\hline 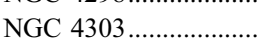 & $4.0 \pm 0.2$ & 17.0 & 6.3 & 1.01 & -9.08 & -1.87 & 9.74 & -0.93 & 55 & Virgo W & -2.78 \\
\hline NGC 4314..................... & $1.0 \pm 0.4$ & 16.0 & 3.8 & -0.18 & -9.97 & -2.57 & $\ldots$ & $\ldots$ & 27 & Coma I & -3.60 \\
\hline NGC 4321........................ & $4.0 \pm 0.3$ & 17.5 & 7.5 & 0.93 & -9.28 & -2.13 & 9.52 & -1.29 & 159 & Virgo A & -3.80 \\
\hline NGC 4383 ..................... & $1.0 \pm 0.9$ & 17.0 & 1.5 & 0.22 & -8.81 & -1.39 & 9.34 & -0.38 & 159 & Virgo A & -3.99 \\
\hline 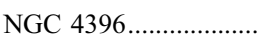 & $6.7 \pm 0.9$ & 17.0 & 3.1 & -0.26 & -8.95 & -2.54 & 9.02 & -0.82 & 159 & Virgo A & $\ldots$ \\
\hline 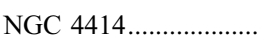 & $5.1 \pm 0.8$ & 18.6 & 2.7 & 0.91 & -9.21 & -1.32 & 9.58 & -0.95 & 27 & Coma I & -5.68 \\
\hline NGC 4413* ................... & $1.9 \pm 1.0$ & 17.0 & 1.8 & -0.37 & -9.27 & -2.17 & 8.35 & -1.29 & 159 & Virgo A & -2.73 \\
\hline NGC 4421...................... & $-0.2 \pm 0.7$ & 17.0 & 2.2 & -1.55 & -10.86 & -3.50 & $\ldots$ & $\ldots$ & 159 & Virgo A & -4.02 \\
\hline NGC 4438....................... & $0.7 \pm 1.6$ & 17.0 & 8.9 & 0.03 & -9.89 & -3.14 & 8.38 & -2.03 & 159 & Virgo A & -1.71 \\
\hline 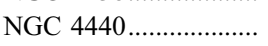 & $1.1 \pm 0.6$ & 17.0 & 1.7 & -1.01 & -10.27 & -2.74 & $\ldots$ & $\ldots$ & 159 & Virgo A & -2.13 \\
\hline 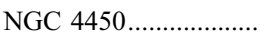 & $2.3 \pm 0.7$ & 17.0 & 5.6 & -0.13 & -10.13 & -2.91 & 8.52 & -1.94 & 159 & Virgo A & -4.44 \\
\hline 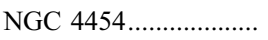 & $0.0 \pm 0.6$ & 34.6 & 2.0 & 0.03 & -9.79 & -2.47 & 8.94 & -1.26 & $\ldots$ & $\ldots$ & -3.44 \\
\hline NGC 4490...................... & $7.0 \pm 0.2$ & 11.0 & 6.3 & 0.66 & -8.85 & -1.84 & 9.82 & -0.98 & 21 & NGC 4258 & -1.16 \\
\hline NGC 4491...................... & $1.1 \pm 0.9$ & 17.0 & 1.6 & -0.38 & -9.25 & -2.09 & $\ldots$ & $\ldots$ & 159 & Virgo A & -4.72 \\
\hline 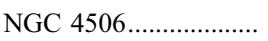 & $1.3 \pm 1.2$ & 17.0 & 1.4 & -0.70 & -9.42 & -2.30 & $\ldots$ & $\ldots$ & 159 & Virgo A & -4.59 \\
\hline 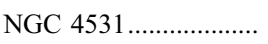 & $-0.3 \pm 2.0$ & 17.0 & 3.0 & -0.60 & -9.93 & -2.85 & 7.43 & -2.33 & 159 & Virgo A & -3.22 \\
\hline 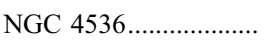 & $4.2 \pm 0.8$ & 15.3 & 7.3 & 0.65 & -9.08 & -2.27 & 9.56 & -0.84 & 55 & Virgo W & -2.47 \\
\hline NGC $4559 \ldots \ldots \ldots \ldots$ & $6.0 \pm 0.4$ & 17.0 & 10.0 & 0.69 & -9.10 & -2.60 & 10.1 & -0.68 & 27 & Coma I & -4.03 \\
\hline NGC 4567....................... & $4.0 \pm 0.3$ & 17.0 & 2.9 & 0.02 & -9.48 & -2.19 & 9.05 & -0.90 & 159 & Virgo A & 0.39 \\
\hline
\end{tabular}


TABLE 1 -Continued

\begin{tabular}{|c|c|c|c|c|c|c|c|c|c|c|c|}
\hline $\begin{array}{l}\text { Object Name }{ }^{a} \\
\text { (1) }\end{array}$ & $\begin{array}{c}T \\
(2)\end{array}$ & $\begin{array}{c}D^{\mathrm{b}} \\
(\mathrm{Mpc}) \\
(3)\end{array}$ & $\begin{array}{c}D_{25} \\
(\operatorname{arcmin}) \\
(4)\end{array}$ & $\begin{array}{c}\log \mathrm{SFR}^{\mathrm{c}} \\
\left(M_{\odot} \mathrm{yr}^{-1}\right) \\
(5)\end{array}$ & $\begin{array}{c}\log \text { sSFR } \\
\left(\mathrm{yr}^{-1}\right) \\
(6)\end{array}$ & $\begin{array}{c}\log \sum_{\mathrm{SFR}} \\
\left(M_{\odot} \mathrm{yr}^{-1} \mathrm{kpc}^{-2}\right) \\
(7)\end{array}$ & $\begin{array}{c}\log M_{\mathrm{H} \text { I }} \\
\left(M_{\odot}\right) \\
(8)\end{array}$ & $\begin{array}{c}\log M_{\mathrm{H}_{\mathrm{I}}} / L_{B} \\
\left(M_{\odot} / L_{\odot}\right) \\
(9)\end{array}$ & $\begin{array}{l}N_{\text {group }} \\
\text { (10) }\end{array}$ & $\begin{array}{l}\text { NOG } \\
\text { Group } \\
(11)\end{array}$ & $\begin{array}{c}f \\
(12)\end{array}$ \\
\hline NGC $4579^{*} \ldots \ldots \ldots \ldots$ & $2.8 \pm 0.7$ & 17.0 & 5.6 & 0.32 & -9.91 & -2.46 & 8.80 & -1.82 & 159 & Virgo A & -3.67 \\
\hline 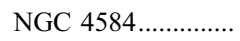 & $1.1 \pm 0.7$ & 17.0 & 1.4 & -0.57 & -9.21 & -2.12 & 7.45 & -1.81 & 159 & Virgo A & -4.73 \\
\hline 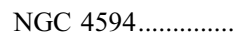 & $1.1 \pm 0.4$ & 9.1 & 8.9 & -0.21 & -10.50 & -2.84 & 8.45 & -2.18 & 6 & NGC 4594 & -5.55 \\
\hline NGC $4736 \ldots \ldots \ldots \ldots . . . . . . .$. & $2.4 \pm 0.7$ & 5.2 & 10.0 & 0.16 & -9.59 & -2.10 & 8.50 & -1.75 & 16 & NGC 4736 & -5.02 \\
\hline NGC $4771 \ldots \ldots \ldots \ldots . . . . . .$. & $6.4 \pm 1.0$ & 17.0 & 3.2 & -0.37 & -9.59 & -2.66 & 8.96 & -0.97 & 21 & Virgo SE; high & -4.10 \\
\hline NGC $4772 \ldots \ldots \ldots \ldots . . . . .$. & $1.1 \pm 0.3$ & 17.0 & 3.6 & -0.07 & -9.55 & -2.46 & 8.99 & -1.02 & 21 & Virgo SE; high & -4.12 \\
\hline NGC $4826^{*} \ldots \ldots \ldots \ldots$ & $2.4 \pm 0.6$ & 17.0 & 9.7 & 0.84 & -9.86 & -2.42 & 9.44 & -1.72 & 2 & NGC 4826 & -6.21 \\
\hline UGC $08313 \ldots \ldots \ldots \ldots$ & $5.0 \pm 0.4$ & 12.1 & 1.4 & -0.95 & -8.71 & -2.20 & 8.14 & -0.72 & 7 & NGC 5194 & -2.41 \\
\hline NGC $5055 \ldots \ldots \ldots \ldots . . . . . .$. & $4.0 \pm 0.2$ & 8.2 & 12.5 & 0.58 & -9.37 & -2.27 & 9.61 & -0.85 & 7 & NGC 5194 & -3.91 \\
\hline NGC 5236* ............. & $5.0 \pm 0.4$ & 4.5 & 13.7 & 0.76 & -9.07 & -1.65 & 9.24 & -1.28 & 10 & Centaurus A & -4.70 \\
\hline 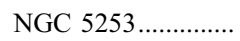 & $7.5 \pm 4.8$ & 3.1 & 4.7 & -0.50 & -8.55 & -1.66 & 7.93 & -1.30 & 10 & Centaurus A & -3.82 \\
\hline NGC 5398.............. & $7.9 \pm 0.8$ & 15.9 & 2.8 & -0.23 & -8.86 & -2.36 & 9.03 & -0.22 & $\ldots$ & $\ldots$ & -5.32 \\
\hline NGC $5457^{*} \ldots \ldots \ldots \ldots$ & $5.9 \pm 0.3$ & 7.5 & 21.6 & 0.90 & -9.01 & -2.34 & 10.0 & -0.61 & 8 & NGC 5457 & -3.04 \\
\hline 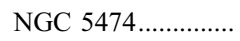 & $6.0 \pm 0.3$ & 6.8 & 2.8 & -0.60 & -8.83 & -1.96 & 8.96 & -0.46 & 8 & NGC 5457 & -3.29 \\
\hline 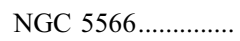 & $1.5 \pm 0.7$ & 22.7 & 5.8 & 0.06 & -10.06 & -3.00 & 9.26 & -1.32 & 5 & NGC 5566 & -1.49 \\
\hline UGC $09215 \ldots \ldots \ldots \ldots$ & $6.4 \pm 0.8$ & 20.8 & 2.2 & -0.06 & -8.84 & -2.21 & 9.29 & -0.43 & 5 & NGC 5566 & -5.15 \\
\hline 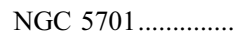 & $-0.4 \pm 0.9$ & 22.8 & 2.2 & -0.21 & -10.03 & -2.44 & 9.69 & -0.49 & 26 & NGC 5746 & -5.81 \\
\hline 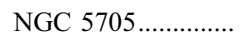 & $6.5 \pm 1.1$ & 26.9 & 1.4 & 0.00 & -8.70 & -2.01 & 9.52 & -0.09 & 26 & NGC 5746 & -1.39 \\
\hline 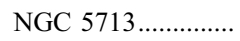 & $4.0 \pm 0.3$ & 26.9 & 2.6 & 0.99 & -8.90 & -1.54 & 9.78 & -0.80 & 26 & NGC 5746 & -3.44 \\
\hline 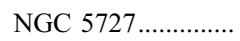 & $7.9 \pm 0.9$ & 24.3 & 1.1 & -0.31 & -8.04 & -1.96 & 9.14 & -0.16 & $\ldots$ & $\ldots$ & -4.11 \\
\hline NGC $5719 . . . \ldots \ldots \ldots . . . . . .$. & $2.4 \pm 0.7$ & 26.9 & 2.8 & 0.63 & -9.30 & -1.95 & 9.79 & -0.21 & 26 & NGC 5746 & -1.95 \\
\hline NGC $5832 \ldots \ldots \ldots \ldots . . . . .$. & $3.1 \pm 0.7$ & 10.6 & 2.4 & -0.80 & -7.81 & -2.42 & 8.88 & -0.21 & $\ldots$ & & $\ldots$ \\
\hline 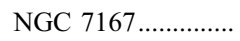 & $5.1 \pm 0.7$ & 35.3 & 1.5 & 0.41 & -9.07 & -1.83 & 9.40 & -0.96 & $\ldots$ & $\ldots$ & -6.93 \\
\hline 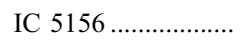 & $2.2 \pm 0.8$ & 37.4 & 2.3 & 0.36 & -9.53 & -2.35 & 9.44 & -0.98 & 12 & PGC 67883 & -4.24 \\
\hline 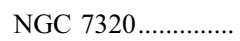 & $6.6 \pm 0.9$ & 13.5 & 2.3 & -0.56 & -8.97 & -2.35 & 8.47 & -1.02 & 4 & PGC 69327 & -3.11 \\
\hline NGC 7331............. & $3.9 \pm 0.4$ & 14.8 & 9.9 & 0.91 & -9.38 & -2.24 & 9.88 & -0.97 & 4 & PGC 69327 & -3.69 \\
\hline NGC $7418 \ldots \ldots \ldots \ldots . . . . . .$. & $5.8 \pm 0.5$ & 18.2 & 3.8 & 0.32 & -9.16 & -2.19 & 9.22 & -0.91 & $\ldots$ & $\ldots$ & -3.38 \\
\hline NGC 7418A............ & $6.5 \pm 1.9$ & 27.6 & 1.9 & 0.04 & -7.81 & -2.23 & 9.58 & -0.28 & $\ldots$ & $\ldots$ & -2.87 \\
\hline 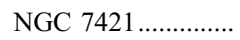 & $3.8 \pm 1.1$ & 23.7 & 2.0 & 0.38 & -9.03 & -1.81 & 8.84 & -1.12 & 5 & PGC 70090 & -3.38 \\
\hline 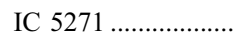 & $3.1 \pm 0.6$ & 22.6 & 2.5 & 0.20 & -9.52 & -2.13 & 9.13 & -1.07 & $\ldots$ & $\ldots$ & -5.60 \\
\hline ESO $406-042^{*} \ldots \ldots$ & $8.8 \pm 0.8$ & 17.2 & 1.5 & -0.73 & -8.17 & -2.40 & 8.43 & -0.57 & $\ldots$ & $\ldots$ & -4.34 \\
\hline NGC $7479 \ldots \ldots \ldots \ldots$ & $4.4 \pm 0.9$ & 34.9 & 3.5 & 1.21 & -8.96 & -1.80 & 9.93 & -0.95 & $\ldots$ & $\ldots$ & -6.39 \\
\hline ESO $407-007^{*} \ldots \ldots$ & $3.1 \pm 0.6$ & 21.0 & 1.9 & -0.22 & -9.27 & -2.27 & 8.82 & -0.69 & 2 & PGC 70582 & -5.23 \\
\hline NGC $7496 \ldots \ldots \ldots \ldots . . . . . . .$. & $3.1 \pm 0.7$ & 20.7 & 3.1 & 0.51 & -9.02 & -1.92 & 9.21 & -0.93 & 13 & PGC 71001 & -4.63 \\
\hline NGC 7496A............. & $9.0 \pm 0.5$ & 20.7 & 1.5 & -0.62 & -8.22 & -2.43 & $\ldots$ & $\ldots$ & $\ldots$ & $\ldots$ & -3.55 \\
\hline ESO $407-009^{*} \ldots \ldots$ & $6.8 \pm 0.9$ & 19.9 & 2.0 & -0.77 & -8.34 & -2.82 & $\ldots$ & $\ldots$ & 2 & PGC 70582 & -5.20 \\
\hline NGC $7552 \ldots \ldots \ldots \ldots . . . . . .$. & $2.4 \pm 0.6$ & 22.3 & 3.9 & 1.32 & -8.72 & -1.38 & 9.55 & -0.95 & 13 & PGC 71001 & -3.52 \\
\hline ESO $407-014^{*} \ldots \ldots$ & $5.0 \pm 0.6$ & 37.0 & 1.5 & 0.30 & -8.88 & -2.03 & 9.53 & -0.51 & $\ldots$ & $\ldots$ & -6.63 \\
\hline NGC $7582 \ldots \ldots \ldots \ldots . . . . . .$. & $2.0 \pm 0.5$ & 22.3 & 6.0 & 1.12 & -9.01 & -1.95 & 9.58 & -0.97 & 13 & PGC 71001 & -1.80 \\
\hline IC $5325 \ldots \ldots \ldots \ldots \ldots$ & $4.2 \pm 0.5$ & 18.7 & 2.8 & 0.31 & -9.26 & -1.96 & 8.82 & -1.28 & 13 & PGC 71001 & -5.69 \\
\hline NGC $7741 \ldots \ldots \ldots \ldots . . . . . .$. & $6.0 \pm 0.4$ & 12.3 & 3.8 & -0.15 & -8.84 & -2.32 & 9.16 & -0.77 & $\ldots$ & $\ldots$ & -5.03 \\
\hline NGC $7793 \ldots \ldots \ldots . . . . . .$. & $7.4 \pm 0.6$ & 2.0 & 9.9 & -0.82 & -9.04 & -2.23 & 8.25 & -0.85 & 13 & Sculptor & $\ldots$ \\
\hline
\end{tabular}

NoтE.-Table 1 is also available in machine-readable form in the electronic edition of the Supplement.

${ }^{a}$ Galaxies noted with an asterisk have a different name in Gil de Paz et al. (this volume).

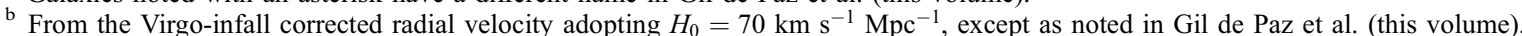

c SFR estimates are derived jointly from TIR and UV luminosities, adopting the calibration of Kennicutt (1998) without any correction for the fractional contribution of diffuse IR emission unrelated to present star formation. 
the tidal perturbation parameter would not necessarily reflect a merger in progress; thus, we also identified those galaxies for which the UV morphology or corollary data indicate evidence for interaction. They are flagged in Table 2, which lists only galaxies classified as XUV-disks.

\subsection{GALEX FUV and NUV Imaging Observations}

GALEX is conducting wide-field $\left(1.25^{\circ}\right)$ imaging and spectroscopic surveys of the sky at far-UV (FUV, 1350-1750 $\AA$ ) and near-UV (NUV, 1750-2750 Å) wavelengths. Details regarding the GALEX surveys and on-orbit performance of the satellite may be found in Martin et al. (2005) and Morrissey et al. (2007).

GALEX obtained FUV and NUV direct imaging observations of our sample targets during primary mission surveys, principally the Nearby Galaxy Survey (NGS; Bianchi et al. 2003; Gil de Paz et al. 2007a) and Medium Imaging Survey (MIS; Martin et al. 2005). Total exposure time for these images is typically $1500 \mathrm{~s}$, leading to $5 \sigma$ point-source limiting magnitudes of $\mathrm{FUV}(\mathrm{AB})=$ 22.6 and $\mathrm{NUV}(\mathrm{AB})=22.7$. All of the images analyzed here were a product of the IR1.1 pipeline. The data are already public and can be obtained from the GALEX archive ${ }^{15}$ (hosted by MAST).

The GALEX PSF is band dependent. FUV images have a typical PSF FWHM of $4.3^{\prime \prime}$, with $80 \%$ of the point-source flux enclosed within $6^{\prime \prime}$. For the NUV band, the FWHM is $\sim 5.3^{\prime \prime}$, with an $80 \%$ enclosed energy diameter of 7 " (Morrissey et al. 2007). The astrometric error is less than $1.1^{\prime \prime}$ for $80 \%$ of sources lying in the central degree of the GALEX field. The photometric zero point appropriate to the FUV and NUV bands was determined using observations of the white dwarf LDS 749B. Uncertainty in the zero points is about $0.07 \mathrm{mag}$ (Morrissey et al. 2007). Residual scatter in the flat field is approximately $7 \%$.

\subsection{DSS-II and SDSS Imagery}

During the classification process, our GALEX imagery was compared to the visible morphology and extent of each target. Second-generation Digitial Sky Survey (DSS-II) imaging data were used for this purpose. With the ESO/ST-ECF DSS program, we retrieved an image centered on each galaxy from the red (IIIaF + RG610) plates, covering 3 times the $D_{25}$ extent of the disk.

Following our classification (see $\S 3$ ), we obtained Sloan Digital Sky Survey (SDSS) DR5 ugriz imagery of confirmed XUVdisk objects whenever possible. SDSS observations were available for $\sim 60 \%$ of the XUV-disks. It would have been preferable to use these photometrically calibrated data for classification purposes, but SDSS is limited to $8000 \mathrm{deg}^{2}$ of the sky.

\section{4. $2 M A S S K_{s}$ Imagery}

To localize the bulk of the old stellar population in our survey galaxies, we extracted 2MASS $K_{S}$ images from the NASA/IPAC Infrared Science Archive (IRSA). Due to the proximity of our sample, many galaxies are included in the 2MASS Large Galaxy Atlas (LGA; Jarrett et al. 2003). This was our preferred source for $K_{s}$ data, as the LGA has already mosaicked individual 2MASS fields to produce a single image covering these galaxies. Of the 189 survey targets analyzed in this paper, 100 were either formally included in the LGA or located well within the LGA field of view for a neighboring object on the sky. $K_{S}$ data for the 89 galaxies unavailable through the LGA were obtained via the Extended Source Catalog (XSC) IRSA interface for the 2MASS All-sky data release, or via a coordinate-based cutout for the small number of galaxies with no XSC entry.

\footnotetext{
15 Available at http://galex.stsci.edu/GR2/.
}

\section{DATA ANALYSIS AND RESULTS}

\subsection{XUV-Disk Classification}

The first discovered XUV-disk galaxies, M83 and NGC 4625 (Thilker et al. 2005a; Gil de Paz et al. 2005), are characterized by obvious UV-bright, spiral structures distributed far beyond the previously recognized outer limit of any recent SF; hence our designation "extended UV-disk" (XUV-disk) galaxy.

However, not all outer disk SF is conspicuous. To enable understanding of the properties of extended ultraviolet galaxies in the framework of a large sample, a classification scheme was needed to define what we consider to be XUV emission. Thus, we first selected a variety of representative galaxies with which to evaluate possible XUV-disk criteria, including the shape of the radial surface brightness and color profiles, the disk surface covering factor of UV-bright clumps, and other indicators such as the $\mathrm{H} \alpha$ /UV flux ratio at outer galactocentric radii. We chose the following scheme to minimize SFH-related bias, avoid pragmatic difficulties in measuring very faint surface brightness profiles, and facilitate application by requiring only commonly available, broadband optical data and GALEX observations.

We define two types of XUV-disk morphology in order to "catch" all apparent cases of spatially extended SF. In particular, the second definition avoids neglecting galaxies that have active SF in outer locations lacking an underlying older disk of appreciable stellar surface density and yet have a well-defined radial limit to such star formation.

We classify XUV-disk galaxies according to the following definitions, which we describe fully in $\S \S 3.1$ and 3.2.

Type 1 XUV-disks show structured, UV-bright emission complexes beyond the anticipated location of the star formation threshold (e.g., M83 in Fig. Set 16; NGC 5055 in Fig. 3, Fig. Set 16); or

Type 2 XUV-disks have blue color (FUV - NIR) within an exceptionally large, outer, and optically LSB portion of the disk (e.g., NGC 2090 in Fig. 4 and Fig. Set 16).

Some galaxies satisfy both definitions and are referred to as mixed-type XUV-disks.

\subsubsection{Type 1 XUV-Disks}

The first of our two XUV-disk definitions has been implemented as follows. We require more than one structured complex of UV-bright emission at positions outside of a centralized, contiguous region contained by a single surface brightness contour corresponding to $\Sigma_{\mathrm{SFR}}=3 \times 10^{-4} M_{\odot} \mathrm{yr}^{-1} \mathrm{kpc}^{-2}$ evaluated at $1 \mathrm{kpc}$ resolution. Galaxies with such morphology are hereafter denoted Type 1 XUV-disks, or alternatively "M83like" objects, adopting a prototype. Additional examples can be seen in Fig. Set 16 of Appendix A. Several pragmatic issues concerning the Type 1 XUV-disk definition are enumerated below.

1. Our choice of the Galactic extinction-corrected UV surface brightness, $\mu_{\mathrm{UV}}\left(\propto \mathrm{SFR}\right.$ surface density, $\left.\Sigma_{\mathrm{SFR}}\right)$, level used to establish the outer clump searching region is motivated both theoretically and observationally. Boissier et al. (2007) demonstrate that for the galaxies of Martin \& Kennicutt (2001) showing a sharp azimuthally drop (in $\mathrm{H} \alpha$ ) attributed to a SF threshold mechanism, typical $\mu_{\mathrm{UV}}$ are near our fiducial level when evaluated at the $\mathrm{H} \alpha$ "edge," even if the edge is merely a consequence of "missing" the outer, short-lived, infrequently-formed $\mathrm{H}$ II regions. Adopting the SFR calibration of Kennicutt (1998), $\Sigma_{\mathrm{SFR}}=$ $3 \times 10^{-4} M_{\odot} \mathrm{yr}^{-1} \mathrm{kpc}^{-2}$ corresponds to $\mu_{\mathrm{FUV}}=27.25 \mathrm{ABmag}$ $\operatorname{arcsec}^{-2}$, or $\mu_{\mathrm{NUV}}=27.35 \mathrm{ABmag} \operatorname{arcsec}^{-2}$ for galaxies only having NUV data. FUV and NUV calibrations are slightly different 
TABLE 2

XUV-Disk Classification Results

\begin{tabular}{|c|c|c|c|c|c|c|c|c|}
\hline $\begin{array}{c}\text { ОвЈест NAME } \\
\text { (1) }\end{array}$ & \multicolumn{2}{|c|}{ TYPE 1} & \multicolumn{5}{|c|}{ TYPE 2} & $\begin{array}{l}\text { INTERACTION COMMENT } \\
\text { (9) }\end{array}$ \\
\hline 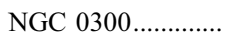 & $\mathrm{Y}$ & FUV & 19.4 & 102.2 & 4.20 & 1.4 & $\mathrm{~N}$ & $\ldots$ \\
\hline NGC $0628 \ldots \ldots \ldots \ldots . . . .$. & $\mathrm{Y}$ & FUV & 179.3 & 1103.3 & 5.09 & 1.9 & $\mathrm{~N}$ & HVCs \\
\hline NGC $0772 \ldots \ldots \ldots \ldots . . .$. & $\mathrm{Y}$ & FUV & 1163.3 & 1262.8 & 0.20 & 1.0 & $\mathrm{~N}$ & asymmetric arm, NGC 770 \\
\hline NGC $1051 \ldots \ldots \ldots \ldots . . .$. & $\mathrm{Y}$ & FUV & 5.9 & 103.0 & 16.26 & 2.3 & $\mathrm{Y}$ & $\ldots$ \\
\hline NGC $1140 \ldots \ldots \ldots \ldots \ldots . . .$. & $\mathrm{N}$ & FUV & 6.6 & 127.2 & 11.15 & 0.4 & Y & pec UV-opt morphology \\
\hline NGC $1512 \ldots \ldots \ldots \ldots . .$. & $\mathrm{Y}$ & FUV & 50.8 & 138.1 & 1.67 & 2.8 & $\mathrm{~N}$ & NGC 1510 \\
\hline NGC $1672 \ldots \ldots \ldots \ldots \ldots$ & $\mathrm{Y}$ & NUV & 116.6 & 646.7 & 4.54 & 2.8 & $\mathrm{~N}$ & warped? \\
\hline NGC $2090 \ldots \ldots \ldots \ldots \ldots . . .$. & $\mathrm{N}$ & FUV & 19.9 & 249.6 & 11.27 & 1.6 & $\mathrm{Y}$ & inner ring \\
\hline ESO $556-012 \ldots \ldots$. & $\mathrm{N}$ & NUV & 4.5 & 203.9 & 23.90 & 0.5 & $\mathrm{Y}$ & $\ldots$ \\
\hline UGC $04390 \ldots \ldots \ldots \ldots$ & $\mathrm{N}$ & FUV & 10.3 & 381.8 & 36.16 & 2.8 & $\mathrm{Y}$ & lopsided UV disk \\
\hline NGC $2710 \ldots \ldots \ldots \ldots . . .$. & $\mathrm{Y}$ & FUV & 79.2 & 262.5 & 2.32 & 2.5 & $\mathrm{~N}$ & P \\
\hline UGC $04800 \ldots \ldots \ldots \ldots$ & $\mathrm{N}$ & FUV & 6.8 & 114.6 & 15.87 & 3.0 & $\mathrm{Y}$ & $\ldots$ \\
\hline NGC $2782 \ldots \ldots \ldots \ldots . . . .$. & $\mathrm{Y}$ & FUV & 121.3 & $\ldots$ & $\ldots$ & $\ldots$ & $\mathrm{N}$ & tidal arms, merger remnant \\
\hline NGC $2841 \ldots \ldots \ldots \ldots . . .$. & $\mathrm{Y}$ & FUV & 123.1 & 281.7 & 1.29 & 5.0 & $\mathrm{~N}$ & outer $\mathrm{H}_{\mathrm{I}}$ ring \\
\hline NGC $3031 \ldots \ldots \ldots \ldots$. & $\mathrm{Y}$ & FUV & 58.8 & 354.0 & 4.95 & 3.6 & $\mathrm{~N}$ & tidal system \\
\hline NGC $3185 \ldots \ldots \ldots \ldots . . .$. & $\mathrm{Y}$ & FUV & 26.9 & 47.2 & 0.63 & 4.8 & $\mathrm{~N}$ & inner UV/opt ring \\
\hline NGC $3198 \ldots \ldots \ldots \ldots . . . .$. & $\mathrm{Y}$ & FUV & 133.7 & 614.8 & 3.59 & 2.4 & $\mathrm{~N}$ & $\mathrm{NE} H$ I tail \\
\hline 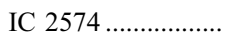 & $\mathrm{N}$ & FUV & 7.2 & 106.0 & 13.76 & -0.3 & $\mathrm{Y}$ & $\ldots$ \\
\hline NGC $3319 \ldots \ldots \ldots \ldots . . . .$. & $\mathrm{N}$ & FUV & 36.1 & 326.9 & 8.05 & 2.0 & $\mathrm{Y}$ & $\mathrm{H}$ I tail, asymmetry from companion \\
\hline NGC $3344 \ldots \ldots \ldots \ldots . . . .$. & $\mathrm{Y}$ & FUV & 28.9 & 190.9 & 5.49 & 1.4 & $\mathrm{~N}$ & asymmetric $\mathrm{H}$ I warp \\
\hline NGC $3359 \ldots \ldots \ldots \ldots . . .$. & $\mathrm{Y}$ & NUV & 102.2 & 604.1 & 4.91 & 1.7 & $\mathrm{~N}$ & accretion via $\mathrm{H}$ I companion \\
\hline NGC $3621 \ldots \ldots \ldots \ldots . . .$. & $\mathrm{Y}$ & FUV & 62.6 & 459.6 & 5.86 & 2.4 & $\mathrm{~N}$ & linear $\mathrm{H}$ I filament across disk \\
\hline NGC $4559 \ldots \ldots \ldots \ldots . . . .$. & $\mathrm{Y}$ & FUV & 212.7 & 1029.9 & 3.75 & 1.9 & $\mathrm{~N}$ & $\ldots$ \\
\hline NGC $4625 \ldots \ldots \ldots \ldots . . .$. & $\mathrm{Y}$ & FUV & 8.7 & 53.8 & 5.17 & 2.2 & $\mathrm{~N}$ & NGC 4618/NGC 4625A \\
\hline NGC $5055 \ldots \ldots \ldots \ldots . . .$. & $\mathrm{Y}$ & FUV & 85.9 & 260.7 & 2.02 & 4.2 & $\mathrm{~N}$ & $\begin{array}{l}\text { TDG? (UGCA 342) and UGC } 8313 \\
\text { (H I filament?) }\end{array}$ \\
\hline UGC $08365 \ldots \ldots \ldots \ldots$ & $\mathrm{N}$ & FUV & 3.9 & 67.0 & 15.65 & 0.6 & $\mathrm{Y}$ & $\ldots$ \\
\hline NGC $5236 \ldots \ldots \ldots \ldots . . . .$. & Y & FUV & 140.3 & $\ldots$ & $\ldots$ & $\ldots$ & $\mathrm{N}$ & NGC 5253 \\
\hline NGC $5457 \ldots \ldots \ldots \ldots . . .$. & $\mathrm{Y}$ & FUV & 338.2 & 1839.2 & 4.39 & 1.4 & $\mathrm{~N}$ & H I lopsided, companions N5477/N5474 \\
\hline 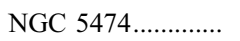 & $\mathrm{Y}$ & FUV & 9.0 & 110.0 & 11.27 & 1.9 & $\mathrm{Y}$ & offset nucleus, NGC 5457 \\
\hline NGC $5705 \ldots \ldots \ldots \ldots . . . .$. & $\mathrm{N}$ & FUV & 23.7 & 322.7 & 12.01 & 0.2 & $\mathrm{Y}$ & companions to SE \\
\hline NGC $5727 \ldots \ldots \ldots \ldots \ldots$ & $\mathrm{N}$ & FUV & 10.3 & 122.3 & 10.85 & 1.9 & $\mathrm{Y}$ & companion \\
\hline NGC $5832 \ldots \ldots \ldots \ldots . . .$. & $\mathrm{Y}$ & NUV & 6.1 & 76.7 & 11.62 & 2.1 & $\mathrm{Y}$ & $\ldots$ \\
\hline UGC $10445 \ldots \ldots \ldots \ldots$ & $\mathrm{Y}$ & FUV & 4.8 & 91.3 & 10.04 & 0.4 & $\mathrm{Y}$ & lopsided UV \\
\hline 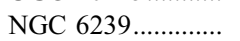 & Y & FUV & 12.6 & 99.4 & 6.26 & 2.5 & $\mathrm{~N}$ & tidal filaments, SDSS $165045.93+424320.4$ \\
\hline NGC $6255 \ldots \ldots \ldots \ldots$ & $\mathrm{N}$ & FUV & 5.2 & 115.3 & 16.40 & 0.9 & Y & $\ldots$ \\
\hline UGC $10791 \ldots \ldots \ldots \ldots$ & $\mathrm{Y}$ & FUV & 7.5 & 49.6 & 5.59 & 3.6 & $\mathrm{~N}$ & NGC 6340 \\
\hline NGC $6902 . . . \ldots \ldots \ldots . . . . . .$. & $\mathrm{Y}$ & FUV & 184.1 & 1092.5 & 4.78 & 2.7 & $\mathrm{~N}$ & inner ring \\
\hline NGC 7418A.................. & $\mathrm{Y}$ & FUV & 1.7 & 527.8 & 22.62 & 0.9 & $\mathrm{Y}$ & NE complex in UV \\
\hline ESO $406-042 \ldots \ldots$. & $\mathrm{N}$ & FUV & 7.8 & 63.2 & 7.11 & 1.5 & $\mathrm{Y}$ & $\ldots$ \\
\hline ESO $407-014 \ldots \ldots$. & $\mathrm{N}$ & FUV & 24.5 & 294.2 & 10.83 & 1.6 & Y & $\ldots$ \\
\hline
\end{tabular}

Notes.-Mixed-type XUV-disks have a "Y" in the Assessment column for both criteria. Colors measured within the LSB zone, UV(AB) - $K_{s}(\mathrm{AB})$, have been corrected for Galactic extinction. 
because we allow for the spectral shape predicted by Starburst 99 for a young population continuously forming stars (e.g., IglesiasParamo et al. 2006). Also, the local star formation threshold H I column density as predicted by Schaye (2004) has been found to correspond to a FUV (NUV) surface brightness very similar to that defining our adopted UV threshold contour (e.g., Thilker et al. 2007). Thus, our threshold contour aims to coarsely delineate where a putative change in the SF properties of the disk may be occurring.

2. We require "structured" emission complexes (e.g., spiral segments, irregularly grouped clumps) to avoid including objects which have faint, but widespread, slowly varying UV emission in their outskirts. Such diffuse emission may result from causes other than recent SF and might be expected in the case of scattered light from dust (Hoopes et al. 2005), or in situ emission from hot core-helium-burning stars. Structured emission complexes suggest a "disk" geometry directly if in the form of spirallike segments. For detecting clumps, we are progressively limited by sensitivity as galaxy distance increases. At a fiducial distance of $10 \mathrm{Mpc}$ and MIS/NGS depth $\left[5 \sigma \sim m_{\mathrm{FUV}}(\mathrm{AB})=22.6\right.$, $\left.m_{\mathrm{NUV}}(\mathrm{AB})=22.7\right]$, GALEX detects clusters having an initial stellar mass of 400, 2600, and $6600 M_{\odot}$ in the FUV band for ages of 3,10, and $100 \mathrm{Myr}$, respectively, whereas nearing the $40 \mathrm{Mpc}$ limit of our sample we are only able to detect $6300 M_{\odot}$ clusters if they are $\sim 3$ Myr old; otherwise the limit rises to $40-100 \times 10^{3} M_{\odot}$. for the more evolved complexes. Given the typical appearance of nearby XUV-disks in our sample, we expect outer structuresto remain resolved for galaxies in the vicinity of our $40 \mathrm{Mpc}$ distance limit.

3. The "UV-bright" requirement refers to the structured clumps individually, not the surrounding, interclump environment. The clumps detected (via visual assessment) in the UV by GALEX must be barely visible or not discernible at $R$-band (checked using the DSS2-red plates). Underlying optical emission can be seen, but must have fundamentally different morphology. For instance, a smooth/diffuse optical background containing clumped UV emission would be allowed.

4. We relax the requirement of more than one detected structured complex in the case where a solitary feature is unquestionably associated with the galaxy (such as a filament composed of several bright clumps, aligned with a known $\mathrm{H}$ I arm).

\subsubsection{Type $2 X U V$-Disks}

Our second XUV-disk definition selects galaxies that are forming stars at an elevated rate over a large area compared to the spatial extent of their evolved stellar population, whether or not they appear to conform to the notions of a star formation threshold (as gauged by the presence of clump complexes extending to extreme outer radii). Objects having such morphology do not resemble the first-known XUV-disk (M83), and were discovered during the present study. NGC 2090 (see Fig. 4, Fig. Set 16) is a prototypical Type 2 XUV-disk. Details of the Type 2 XUVdisk definition are given below.

To quantitatively identify Type 2 XUV-disk structures, we defined a "low surface brightness (LSB) zone" as the region contained within the anticipated SF threshold $\left(\Sigma_{\mathrm{SFR}}\right)$ contour, yet outside a $K_{s}$-band contour enclosing $80 \%$ of the total $K_{s}$ luminosity of the galaxy. The FUV $-K_{s}$ color of this optically LSB zone can then be used as a metric of the luminosity-weighted age of the associated stellar population. If the average UV(AB)$K_{s}(\mathrm{AB})$ of the LSB zone is $\leq 4$, indicating the relative importance of recent star formation with respect to the underlying disk and the area of the zone, $S(\mathrm{LSB})$, is at least 7 times the enclosed area
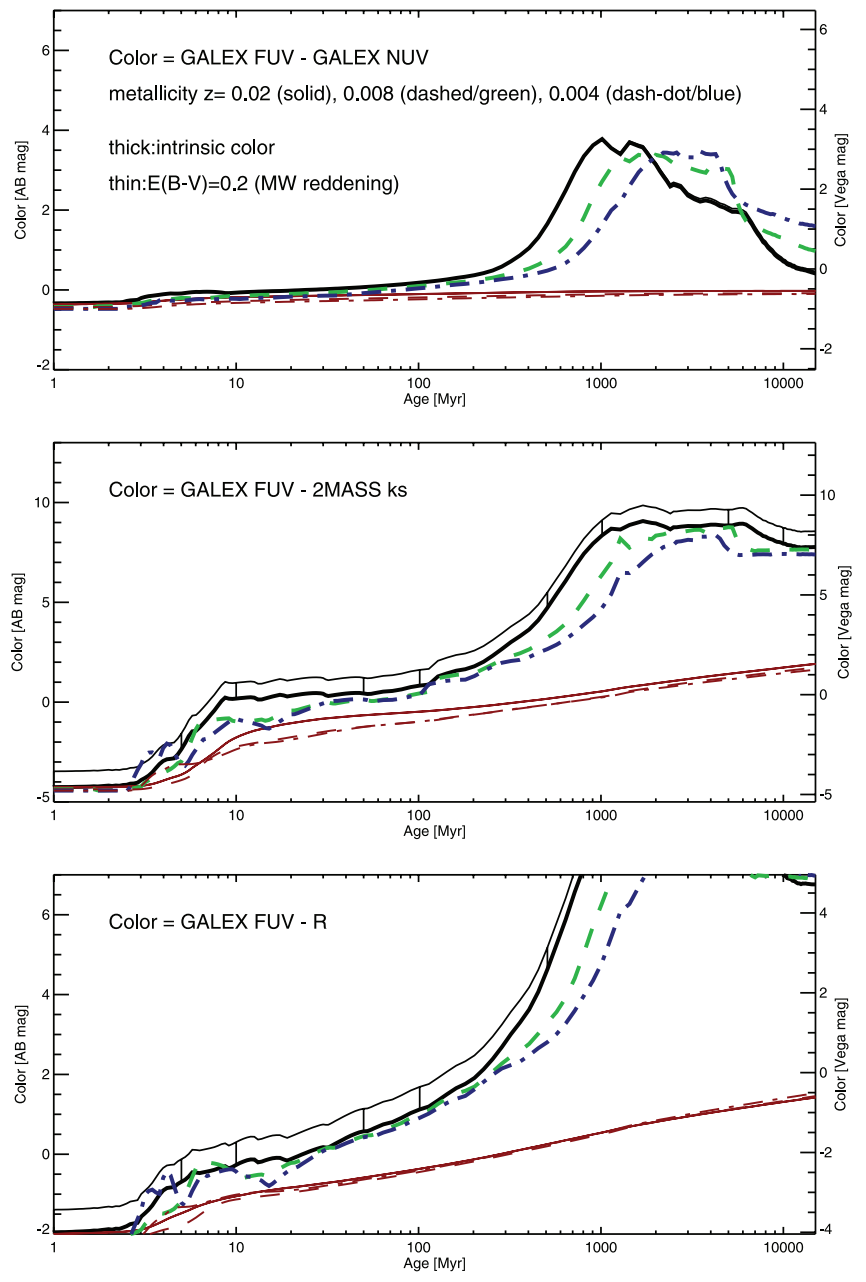

FIG. 1.-Variation of FUV - NUV and FUV-optical colors as a function of age for Bruzual \& Charlot (2003) models of an instantaneous starburst and continuous star formation (red lines). In all panels, we show three different metallicities $(Z=0.02$, solid line; $Z=0.008$, dashed green line; and $Z=0.004$, dash-dotted blue line). For the instantaneous starburst, solar metallicity case we also plot (with a thin line) reddened colors for $E(B-V)=0.2$ and the MW attentuation law $\left(R_{V}=3.1\right)$. Colors are specified in both ABmag and Vegamag systems. The panels correspond to FUV - NUV (top), FUV $-K_{s}$ (middle), and FUV $-R$ (bottom). The FUV $-K_{s}$ model colors imply that our requirement that FUV $(\mathrm{AB})-K_{s}(\mathrm{AB}) \leq 4$ for Type $2 \mathrm{XUV}$-disks corresponds to a burst population younger than $\sim 500 \mathrm{Myr}$, provided that FUV - NUV is significantly redder than about -0.5 (excluding a much older continuous star formation event).

of the $K_{s}$ contour, $S\left(K_{80}\right)$, then we classify the galaxy as an XUV-disk.

1. In Figure 1 we show Bruzual \& Charlot (2003) population synthesis model colors versus age for three metallicities in two SFH scenarios, an instantaneous starburst (Fig. 1a) and continuous star formation (Fig. 1b). All models assume a Chabrier IMF (Chabrier 2003). Our adopted FUV $-K_{S}$ cutoff was determined empirically (see the discussion of Fig. 2 below), but corresponds to an instantaneous burst of age less than $\sim 500 \mathrm{Myr}$ in the case of solar metallicity $(\sim 1 \mathrm{Gyr}$ for $Z=0.004)$. A much older epoch of continuous star formation is also consistent with some of the bluest FUV $-K_{s}$ colors measured within LSB zones. However, this scenario generally can be excluded on the basis of FUV - NUV (with observed values too red [slightly positive] for the continuous SF model). FUV $-K_{s}$ may also be interpreted as a metric of the specific star formation rate (sSFR; MuńozMateos et al. 2007), although the precise calibration of the relation 


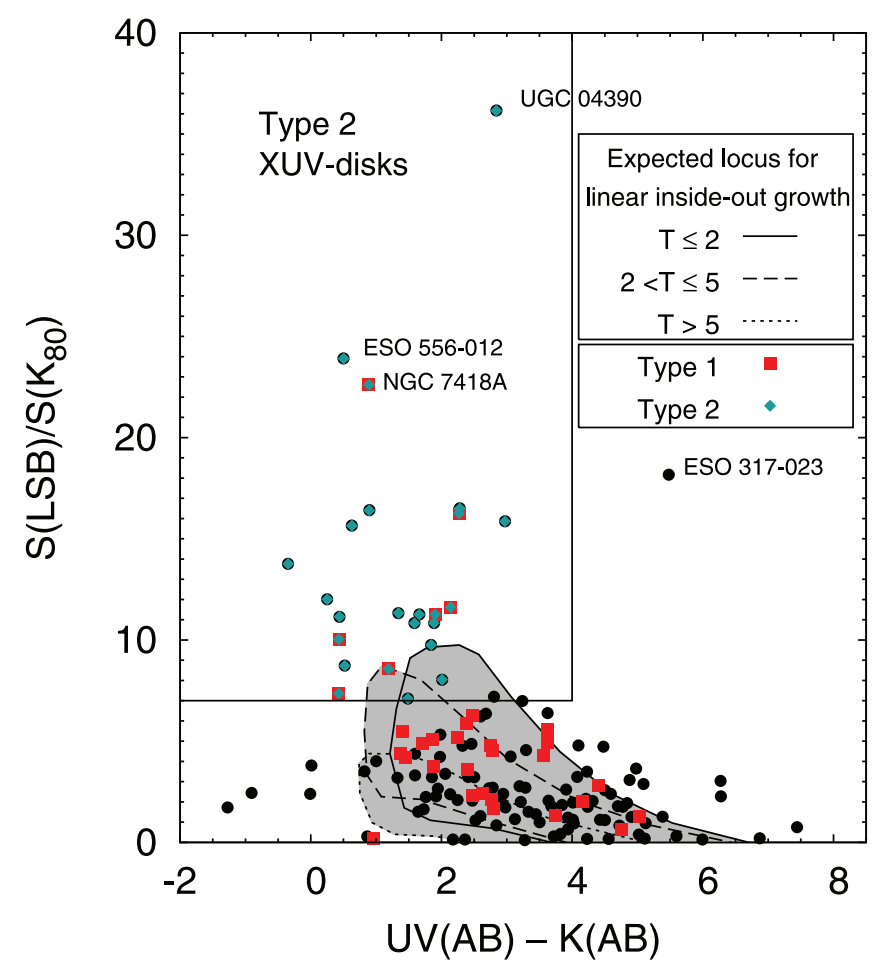

FIG. 2.- LSB zone to $K_{s}$-band $80 \%$ effective area ratio, $S(\mathrm{LSB}) / S\left(K_{80}\right)$, vs. FUV $-K_{s}\left[\mathrm{NUV}-K_{s}\right]$ measured in the LSB zone. Note the excess of objects having blue color and a comparatively large LSB zone. We empirically define the objects having $S(\mathrm{LSB}) / S\left(K_{80}\right) \geq 7$ and $\mathrm{FUV}(\mathrm{AB})-K_{s}(\mathrm{AB}) \leq 4$ to be Type 2 (or possibly mixed-type) XUV-disk galaxies. Symbols are red squares for Type 1 galaxies, blue diamonds for Type 2 objects, and black circles for other galaxies in the sample. We overplot the predicted locus of "normal" galaxies growing from inside-out for three categories in $T$, according to the models of MunozMateos et al. (2007). The relative spatial extent of the star-forming LSB zone in Type 2 XUV-disk galaxies is clearly extreme even for the inside-out disk building process. The single black data point inside the area designated at Type $2 \mathrm{XUV}$ disks is NGC 7479, which was classified as non-XUV because it fell much closer to the normal galaxies in the plot than the other Type 2 XUV-disks.

depends on the type of star formation history assumed. Figure 1 also shows predicted values of FUV $-R$, which demonstrate the expected overall faintness of visible emission in XUV-disks, relative to their UV appearance.

2. The distribution of relative size, $S(\mathrm{LSB}) / S\left(K_{80}\right)$, versus Galactic extinction-corrected FUV(AB) $-K_{s}(\mathrm{AB})$ measured in the LSB zone [NUV $(\mathrm{AB})-K_{s}(\mathrm{AB})$ where FUV data are unavailable] is presented in Figure 2. Note the tail of objects with large relative size and blue FUV $-K_{s}$ for the LSB zone. Lines segregate the objects classified as Type 2 XUV-disks (or mixed-type XUV-disks). The influence of the bulge component on the $K_{80}$ contour most likely introduces a bias encouraging the classification of early-type spirals as Type 2 XUV disks. However, the observed distribution of points in Figure 2 shows an abrupt dropoff just below our cutoff of $S(\mathrm{LSB}) / S\left(K_{80}\right)=7$, suggesting that the Type 2 disks are extreme in terms of their disk formation history, and $T$-type dependent bulges influence our classification minimally. Our Type 2 classification limits were determined empirically, but after the fact we compared our measured characteristics to model predictions describing inside-out disk formation (Muńoz-Mateos et al. 2007). The anticipated locus of "normal" galaxies of varied $T$ is shown by the shaded regions in Figure 2. Although there is minor overlap between the Type 2 XUV-disk class and the model predictions, this analysis demonstrates that Type 2 objects have an anomalously large LSB zone even for disks assumed to be forming inside-out in a smooth continuous manner. Conversely, the measured $S(\mathrm{LSB}) / S\left(K_{80}\right)$ and color of the LSB zone for non-Type-2 galaxies generally agrees well with the model.

3. Our Type 2 XUV-disk definition does not exclude objects in which the LSB zone is optically visible, even if UV-bright clumps can been seen in other bands.

\subsubsection{Mixed-Type Disks and a Caveat}

As mentioned above, some objects satisfy both Type 1 and Type 2 XUV-disk definitions. We refer to these galaxies as "mixed-type" XUV-disks.

It is also worth noting that the continuum of properties found in our sample is such that there are also galaxies which fail the Type 2 XUV-disk definition but yet have a large, blue outer disk. An example is NGC 4625, which has an optically LSB, outer disk which is blue enough (FUV $\left.-K_{s}=2.2\right)$, but with a relative size $S(\mathrm{LSB}) / S\left(K_{80}\right)=5.17$ that does not meet our cutoff. However, significant XUV-emission is distributed beyond the UV threshold contour for NGC 4625, qualifying it as an XUV-disk according to our Type 1 definition. Except for the typical $\Sigma_{\text {SFR }}$, this outermost SF appears similar to that occurring within the UV threshold contour. If these regions had been included in the LSB zone, NGC 4625 would also have met the Type 2 definition. The purpose of making this point is to emphasize that even objects belonging exclusively to a particular XUV-disk type in our analysis may (to a lesser degree) have some of the characteristics of the other type. Furthermore, it suggests to us a possible future approach to unifying the classification scheme, if a robust means of extending the limits of the LSB zone definition (into lower $\Sigma_{\mathrm{SFR}}$ areas) could be devised.

\subsubsection{Application of the Classification Scheme}

In this section we present figures illustrating the method of XUV-disk galaxy classification. Note that our identification of Type 1 extended UV emission remains subjective, so this section is also intended to convey a sense of the decision-making process. Our Type 2 definition is fully automated.

NGC 5055 (M63) is a SA(rs)bc galaxy which shows obvious XUV emission of the "M83-like" variety selected by our Type 1 definition. It has a significant degree of structured, UV-bright emission outside an FUV "threshold" contour. Figure 3 shows the GALEX FUV data and a false-color image using the $2 \mathrm{MASS}-K_{s}$, DSS2-red, and DSS2-blue data, in addition to the FUV and $K_{S}$ isophotal contours described above. Recall that the FUV contour defines a region outside of which multiple structured, UV-bright emission complexes are considered as support for XUV-disk classification. The area between the $K_{s}$ and FUV contours is where we measure the LSB zone color. In the case of NGC 5055 the zone is too small and red to satisfy our second XUV-disk definition.

NGC 2090, a Type 2 XUV-disk (Fig. 4), is a SA:(rs)b galaxy which has only minimal UV emission located beyond the FUV threshold contour, yet exhibits very blue colors over most of the disk inside this limit. It has an expanded LSB zone, extending over an area more than an order of magnitude larger than the effective (80\%) size of the old stellar disk. Incidentally, NGC 2090 is a target for which our Type $1 \mathrm{XUV}$-disk definition is somewhat ambiguous. UV emission is found beyond the threshold contour (green line); however, the observed structure (on the west side of the disk) is a smooth continuation of the disk morphology just inside the contour. This type of "fluff" at the disk edge was ignored in our classification analysis, given that we are only able to see such structure in relatively nearby galaxies.

Figure 5 shows an example of a galaxy not classified as an XUV-disk. This object, NGC 7418, is a typical SAB(rs)cd galaxy 


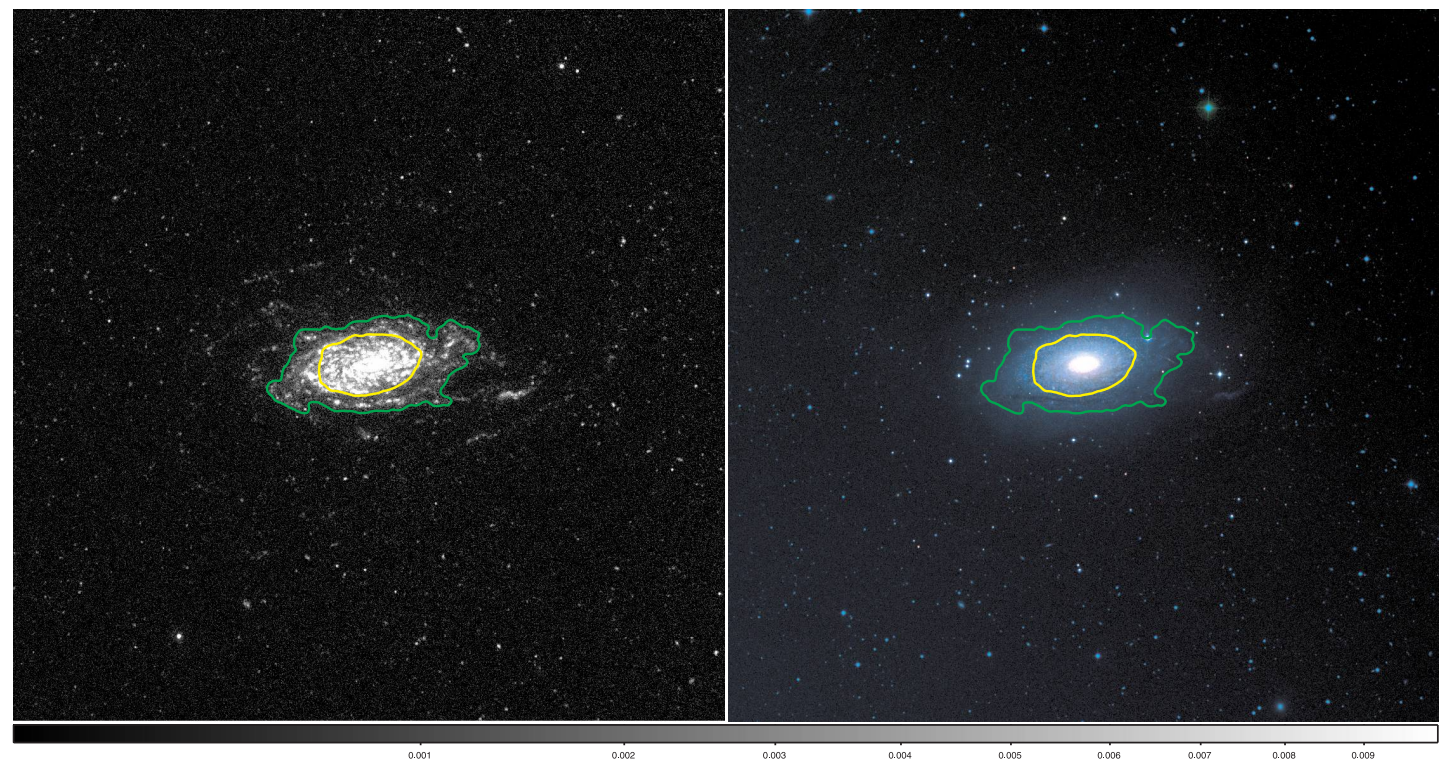

FIG. 3.-FUV-NIR imagery and classification contours for XUV-disk galaxy NGC 5055 (M63), a prototype for our Type 1 class. On the left we show the GALEX FUV image of the galaxy. On the right we show the 2MASS $K_{s}$-band, DSS2-red, and DSS2-blue imaging (as RGB channels) for an identical field of view ( $3 D_{25}=$ $37.5^{\prime}=89.4 \mathrm{kpc}$ at $8.2 \mathrm{Mpc}$ ). Contours are the same on both images. At the green line, the FUV surface brightness (corrected for Galactic foreground extinction and measured at $1 \mathrm{kpc}$ resolution) is $\mu_{\mathrm{FUV}}=27.25 \mathrm{AB}$ mag arcsec${ }^{-2}$. This is the position at which (apparent?) star formation threshold mechanisms are thought to become important. The yellow contour encloses $80 \%$ of the $K_{s}$-band luminosity of the galaxy, defining the effective extent for the old stellar population. Note the structured UV-bright emission features beyond the green (UV) contour, which give this galaxy the Type 1 XUV-disk designation.

which has an obvious color gradient as a function of galactocentric distance, $r_{g}\left(\mathrm{FUV}-K_{s}\right.$ decreasing with $r_{g}$ ). The form of this gradient is such that in the outermost part of the disk FUV $\left.-K_{s} \leq 4\right)$. However, the spatial extent of this blue "fringe" is small, and $S(\mathrm{LSB}) / S\left(K_{80}\right)<1$. Figure 5 also illustrates the difficulty of distinguishing distant background galaxies from bona fide outer disk clusters in the case of isolated UV clumps, not belonging to a structure such as a clump complex or filamentary feature. A few examples of isolated UV-bright sources with little or no optical emission can also be seen in Figure 5. Recall that our Type 1 definition requires structured UV emission features and therefore may (lacking substantially higher resolution UV observations) miss some galaxies that do support sporadic outer disk SF at such a low level that it occurs only in isolated, unresolved clumps. Such missed XUV-disk galaxies may be represented by the emission-line dot ("EL Dot") phenomenon (Ryan-Weber et al. 2004; Werk et al. 2007).

Figure 6 shows a second example of a galaxy not classified as an XUV-disk. NGC 4736, a nearby (R)SA(r)ab; Seyfert 2 LINER galaxy, has an inner SF ring contained by the $K_{s}$-band contour ( yellow line), complemented by an extensive one-arm spiral composed of UV-bright clumps starting at the circumnuclear

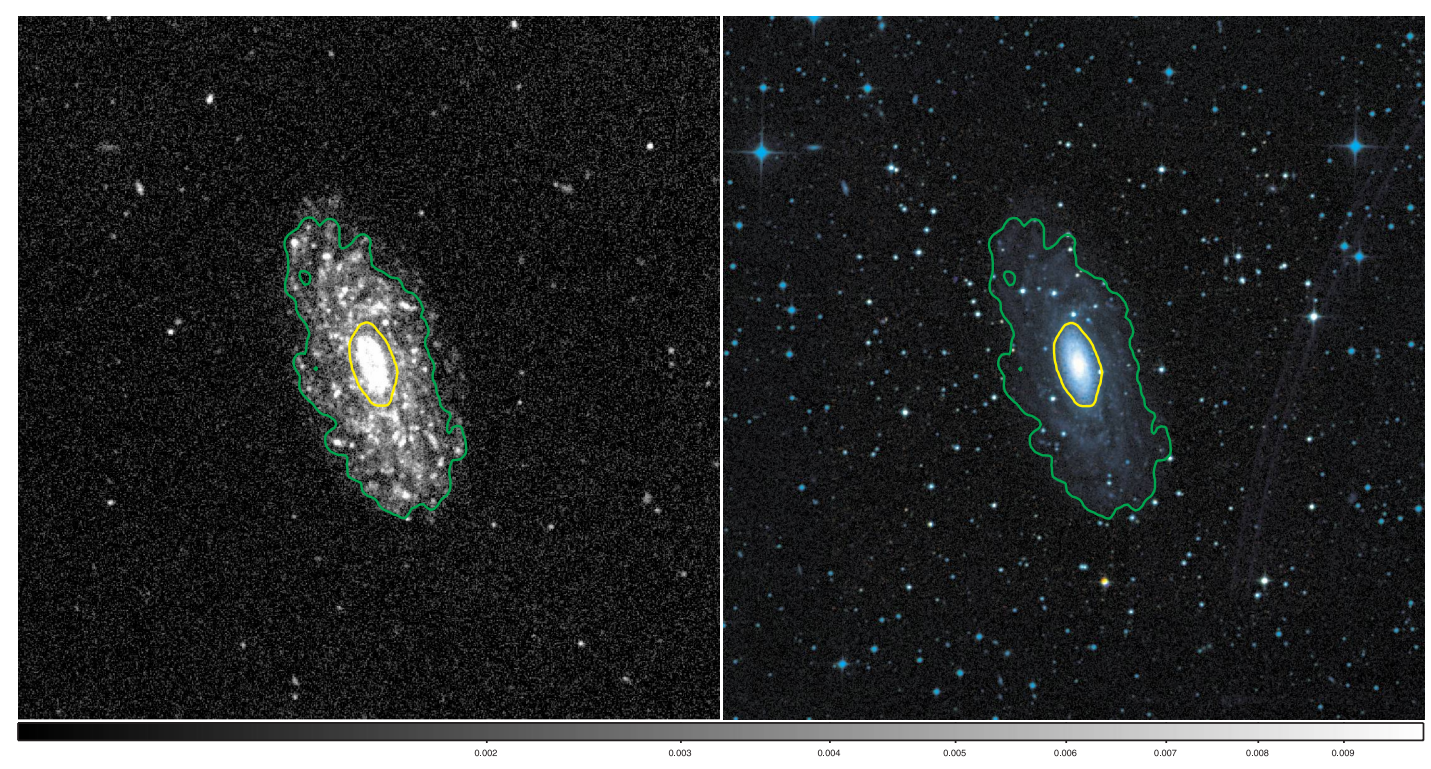

FIG. 4.-FUV-NIR imagery and classification contours for Type 2 XUV-disk galaxy NGC 2090. We observe a rather large blue LSB zone, which dominates the spatial extent of the galaxy despite being of low (optical) surface brightness. The image passbands and contour types are identical to those of Fig. 3 . The field of view spans $3 D_{25}=12.9^{\prime}=42.4 \mathrm{kpc}$ at $11.3 \mathrm{Mpc}$. 


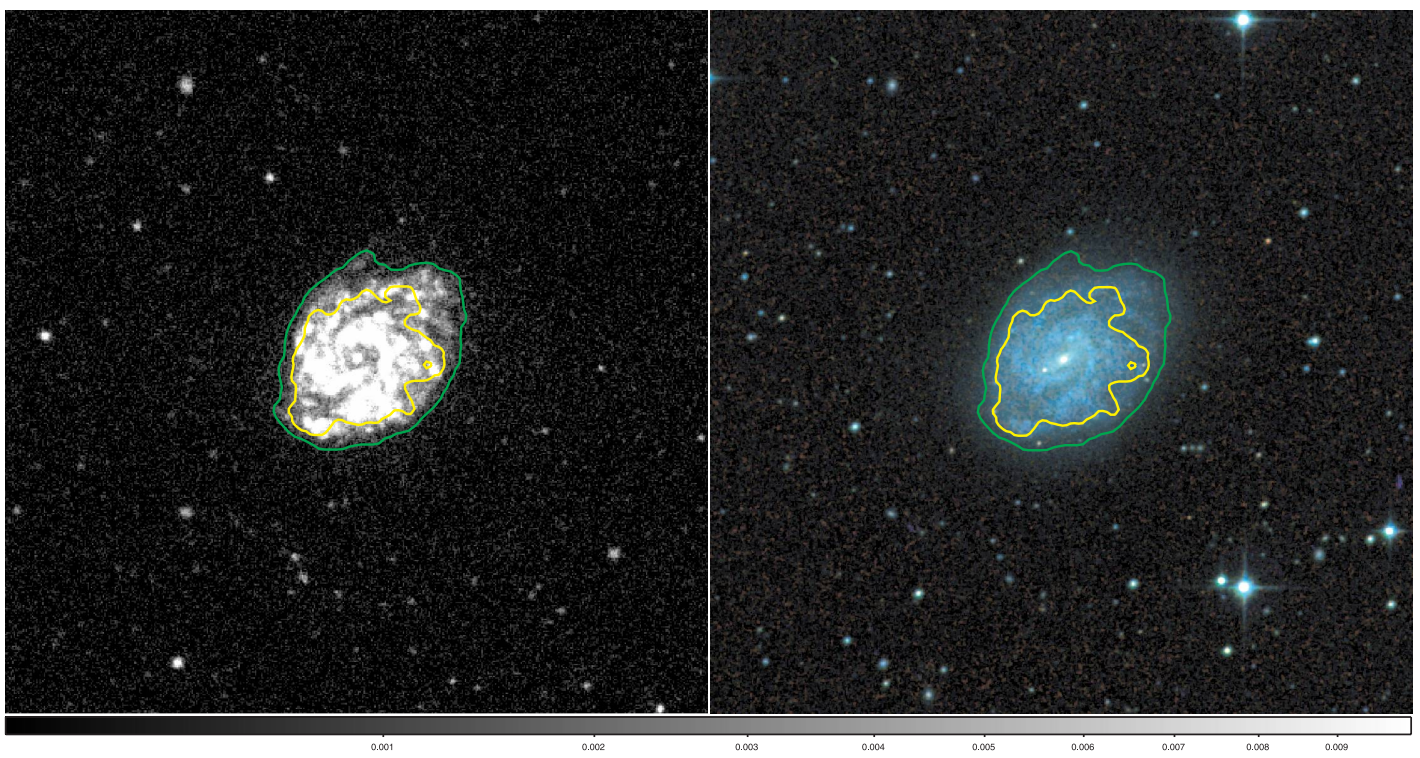

FIG. 5.-FUV-NIR imagery and classification contours for non-XUV-disk galaxy NGC 7418, presented as an example of a target having a blue edge that is not extended enough to be considered significant for classification purposes. Furthermore, no structured UV emission can be confidently associated with the target beyond the FUV "threshold" contour. NGC 7418, an SAB(rs)cd galaxy, has FUV $-K_{s}=2.8$ in the LSB zone, but $S(\mathrm{LSB}) / S\left(K_{80}\right)=0.8$. The image passbands and contour types are identical to those of Fig. 3. The field of view spans $3 D_{25}=11.4^{\prime}=60.3 \mathrm{kpc}$ at $18.2 \mathrm{Mpc}$.

ring and eventually reaching the outer disk (beyond the green contour). However, optical (DSS2-red) imagery clearly shows a matching structure of moderate surface brightness emission. Our Type 1 XUV-disk definition allows for optical emission observed cospatially with outer disk UV-clumps, but we require a clearly different form for the evolved stellar population traced by the longer wavelength data. NGC 4736 is a borderline case falling between tidally induced Type 1 XUV-disks such as NGC 1512 with LSB underlying optical emission, and nonXUV-disk "train wreck" objects such as the Antennae (NGC 4038/39), with in situ SF formation occurring inside luminous stellar tidal tails (Hibbard et al. 2005). Because the future disk-like nature of extreme, equal-mass mergers like NGC 4038/4039 is questionable, we sought to keep them out of the XUV-disk class. The similar appearance of moderate surface brightness optical emission having embedded UV clumps found in NGC 4736 (vs. the Antennae), led us to group them together. Even though there is no direct evidence that NGC 4736 has experienced a significant recent merger, it could have undergone merging in the distant past. A few other galaxies in our sample have UV/optical morphology similar to NGC 4736. In particular, NGC 1068 and NGC 1291 show UV-bright SF complexes within optically matched outer regions reminiscent of merger activity. Might these galaxies be examples of objects which have experienced

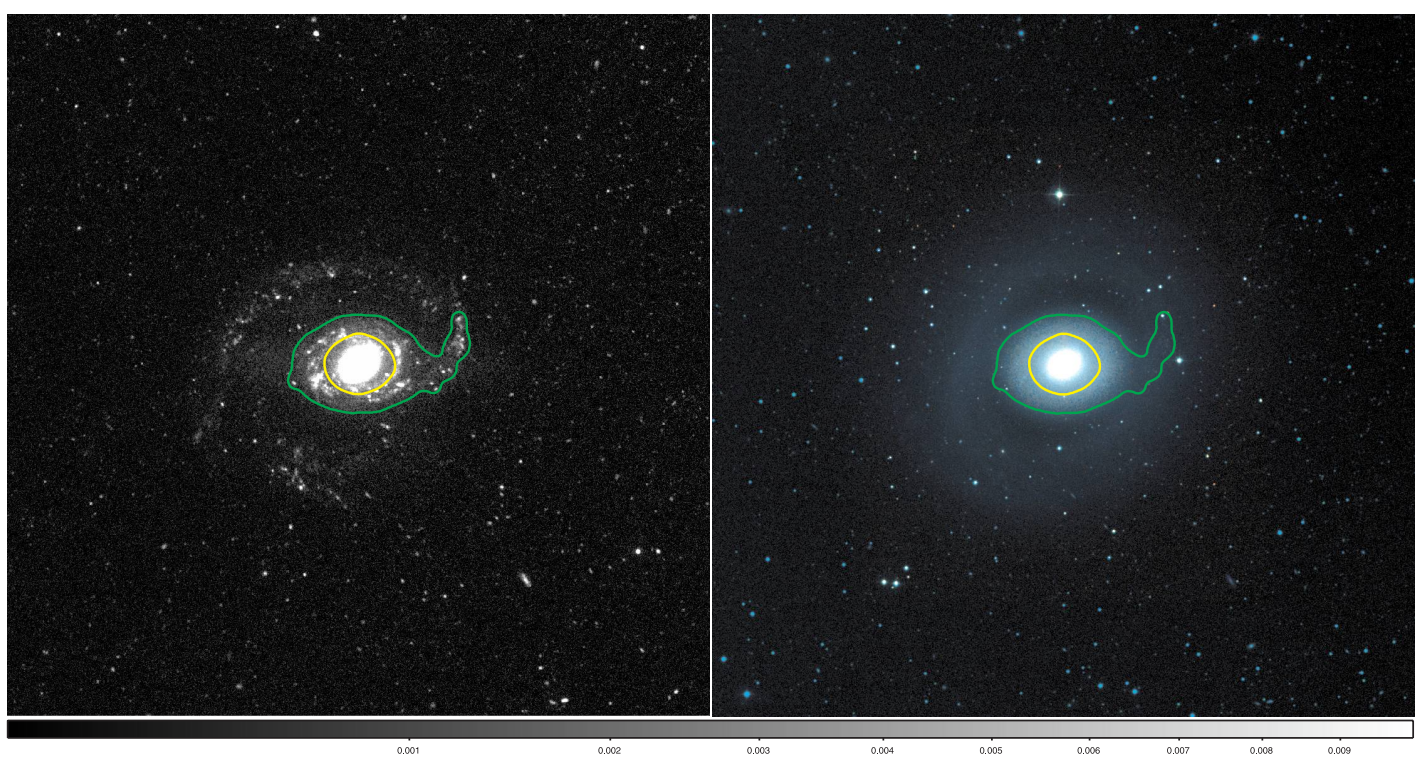

FIG. 6.-FUV-NIR imagery and classification contours for non-XUV-disk galaxy NGC 4736 [M94, type (R)SA(r)ab;Sy2 LINER], presented as an example of a target having significant, structured, UV-only clumps beyond the threshold radius, but with relatively HSB optical structure in nearly the same form. The LSB zone in NGC 4736 is typical, with $S(\mathrm{LSB}) / S\left(K_{80}\right)=2.9$ and (FUV $\left.-K_{s}=5.1\right)$. The image passbands and contour types are identical to those of Fig. 3 . The field of view spans $3 D_{25}=30.0^{\prime}=136 \mathrm{kpc}$ at $5.2 \mathrm{Mpc}$. 
recurrent XUV-disk episodes and built up an older stellar population at large galactocentric distance? Assuming that repeated episodes of extended SF are unrelated to each other, they should eventually form a quasi-smooth outer disk with minimal resemblance to the most recent SF episode, making such a hypothesis unlikely.

\subsection{Incidence of XUV-Disk Star Formation}

Based on the classification scheme outlined above, we have identified a set of 54 XUV-disk galaxies selected from our current sample of 189 targets. This shows that XUV-disk SF is a common evolutionary process in the spiral galaxy population. Our catalog of XUV-disks also allows us to look for trends related to Hubble type and other galaxy properties.

We found 30 objects satisfying only the Type 1 XUV-disk definition, 17 objects satisfying only the Type 2 definition, and 7 galaxies matching both definitions (mixed-type). Considering the union of the first and last groups, we find that approximately $220 \%$ (37/189) of the nearby spiral galaxy population is supporting massive star formation in outer disk locales beyond the classically expected star formation threshold. We emphasize that this estimate of incidence for M83-like XUV-disk structure in our sample is a lower limit. The ease of detecting the starforming background galaxy population with GALEX makes it difficult to rule out activity occurring in very isolated complexes consisting of a single clump (at our $\sim 5^{\prime \prime}$ resolution). (Such background contamination is less of a problem for $\mathrm{H} \alpha$-based surveys, which can select line emission at the target galaxy's velocity.) Also note that some outer, low-mass clump complexes probably remain undetected in galaxies near our $40 \mathrm{Mpc}$ distance limit. Indeed, cataloged Type 1 XUV-disks are not distributed evenly throughout our survey volume. Ignoring Virgo Cluster members (which set the median distance of the sample), we find that 17 of 71 galaxies closer than $16 \mathrm{Mpc}$ meet our Type 1 definition, whereas only 8 of 70 beyond $20 \mathrm{Mpc}$ are classified as such. This implies that we have likely missed a few galaxies having very inconspicuous outer disk SF complexes (similar to NGC 300) in the more distant subset of our sample. Regarding the objects having an exceptionally large, blue LSB zone (Type 2 and mixedtype XUV-disk galaxies), we find that they comprise about $13 \%$ $(24 / 189)$ of our sample. In our sample, distance effects do not seem to influence the identification of Type 2 structures, given that 8 of 71 galaxies at $D<16 \mathrm{Mpc}$ satisfy the Type 2 definition, compared to 10 of 70 beyond $20 \mathrm{Mpc}$. As noted above, the overall incidence of XUV-disk star formation is $(54 / 189) \gtrsim 29 \%$ in the local universe.

\subsection{XUV-Disk SF in Relation to Galaxy Properties and Environment}

\subsubsection{Distribution in Hubble Type and $L_{K}$}

In Figure $7 a$ we show a histogram of $T$-type for the entire sample and both groups of XUV disks. Our survey sample is clearly weighted toward intermediate-type spirals. Type 1 XUVdisks are distributed over a very broad range in $T$, with galaxies occupying nearly every bin with the exception of the extrema in $T$ (for which there are very few objects in our sample). We refrain from measuring relative incidence or internal strength of XUV features for Type 1 XUV-disk galaxies as a function of $T$, due to the limited number of objects detected. Kolmogorov-Smirnov (K-S) testing indicates that the $T$ distribution of Type 1 XUVdisks matches that of the entire sample at the $82 \%$ confidence level. The distribution of Type 2 XUV-disk galaxies is more skewed toward late-type spirals, with the vast majority of detections having $T>5$. With K-S testing, we can exclude ( $>99 \%$
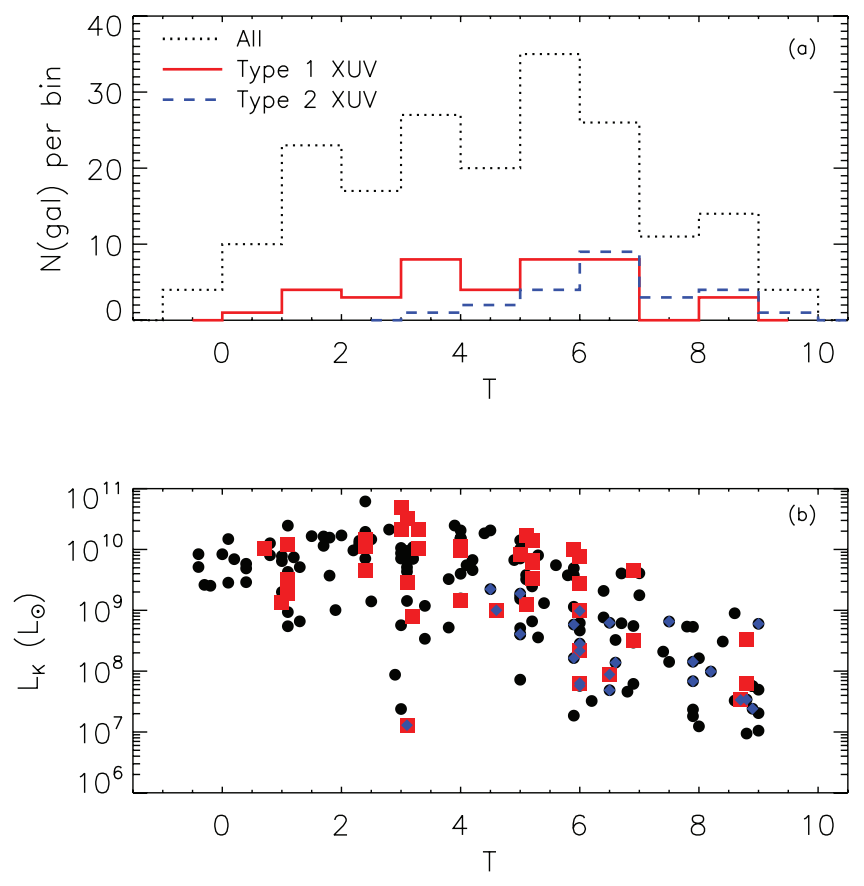

FIG. 7.- (a) Distribution of Hubble type (T) for all galaxies in our sample (black dotted line), in comparison to Type 1 XUV-disks (red solid line) and Type 2 XUV-disks (blue dashed line). (b) $L_{K}$ vs. Hubble type, using the same color coding as in $(a)$. In addition, Type 1 XUV-disks have been drawn with a square and Type 2 XUV disks with a diamond, allowing for identification of galaxies meeting one (or both) of our XUV criteria.

confidence) the possibility that Type 2 XUV-disks are drawn randomly from the survey sample. Note that our Type 2 definition for XUV-disk classification does not highlight the entire population of very late-type spirals as being peculiar, despite the trend for globally integrated bluer FUV $-K_{s}$ with increasing $T$ (Gil de Paz et al. 2007a). In fact, most sample galaxies with $T \geq 8$ are not XUV-disks, although this statement is complicated by the disappearance of a bulge component in Sm galaxies which could influence our LSB zone implementation.

The nearly ubiquitous observed distribution in $T$ for Type 1 objects may suggest that the presence of a Type 1 XUV-disk has more to do with the environment or interaction/accretion history of a galaxy than the host galaxy itself.

Figure $7 b$ shows the distribution of our entire sample and that of XUV-disks, color coded according to type, in the $T-L_{K}$ plane. Here $L_{K}$ is a reliable, nearly extinction-free tracer for the stellar mass of a galaxy (Gavazzi et al. 1996; Bell \& de Jong 2001). There is no apparent offset between the XUV-disk galaxies and the overall population, leaving open the possibility of episodic XUV-disk star formation (with XUV active objects drawn from the same parent sample).

There is a rough separation of the XUV-disk varieties in terms of $L_{K}$. This division is near $L_{K}=10^{9} L_{\odot}$. Assuming a mass-tolight ratio of 0.8 (Dale et al. 2007), it would appear that galaxies are unable to support (or already have undergone?) a (Type 2) galaxy-wide transformational burst of star formation when their stellar mass becomes on the order of $10^{9} M_{\odot}$ or greater. Boissier et al. (2001) demonstrated that more massive galaxies have lower gas fraction, suggesting that Type 2 XUV-disks are formed only when the gas reservoir is large with respect to the stellar population.

\subsubsection{Correlation with SFR Measures and Gas Content}

Following up on the observation that Type 2 XUV-disks are preferentially late-type spirals whereas Type 1 XUV-disks occur 


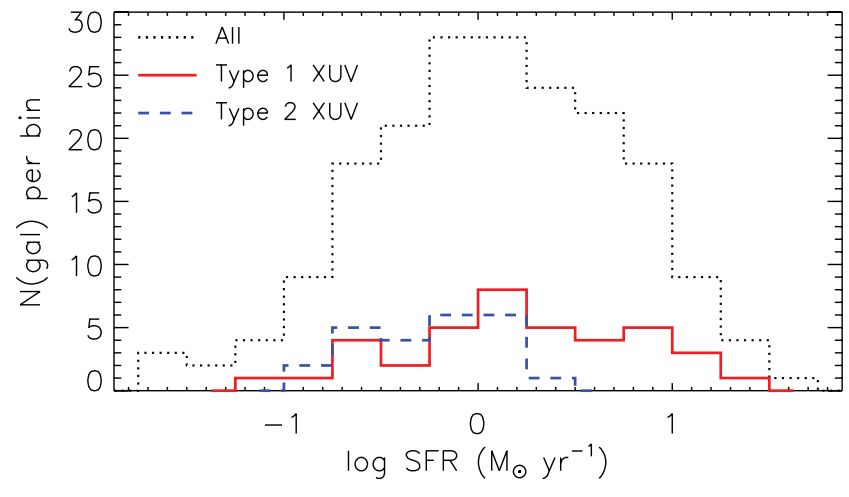

FIG. 8.-Distribution of globally averaged SFR for the survey sample (black dotted line) and for galaxies classified as XUV-disks (red solid line, Type 1; blue dashed line, Type 2). SFR has been estimated on the basis of UV and IR luminosity jointly.

in systems of all Hubble type, we sought to check for correlations with other galaxy parameters (e.g., SFR, sSFR, SFR/M $M_{\mathrm{H}_{\mathrm{I}}}$ ). Although these variables are correlated with $T$ and $L_{K}$, XUV disks may still deviate from the trend of the overall galaxy population.

Figure 8 presents a histogram illustrating the distribution of integrated SFR for the entire sample, in comparison to the distribution for XUV disks of each type. We note that despite seemingly prodigious SF (in terms of the distinctive UV-NIR appearance and generally widespread outer disk SF leading to their selection), Type 2 XUV-disks appear to have a maximum SFR of a few $M_{\odot} \mathrm{yr}^{-1}$. Although the Type 2 outer disks are effectively forming now, they are not "bursting" in the manner of nuclear starbursts characterized by remarkably high star formation efficiency. The integrated SFR values of Type 1 XUV-disks are dominated by $\mathrm{SF}$ in the main disk, rather than the localized cluster complexes throughout the outer disk, making the distribution of SFR for these objects less relevant. It is important to emphasize that in all XUV-disk galaxies, regardless of type, the fractional contribution to the total SFR from SF outside of our estimated threshold contour is low. The widespread LSB zone SF in Type 2 XUV- disks is occurring inside the threshold, and thus should not be compared directly to the level of XUV-disk SF in Type 1 objects.

When cast into a specific star formation rate, the remarkable short-term history of Type 2 XUV-disks becomes apparent. Figure $9 a$ plots sSFR versus $L_{K}$ and illustrates that, although the integrated SFR may be modest, such activity is sufficient to completely form the stellar mass of a Type 2 XUV disk generally in less than $1 \mathrm{Gyr}$. The specific star formation rate here refers to the entire galaxy. Within the blue LSB zone, the specific SFR is even higher. Because we have plotted the galaxy-averaged quantity in Figure $9 a$, the Type 1 XUV disks appear be very similar to the rest of the sample. However, as already noted for Type 2 XUV disks, the sSFR of the outermost disk can be substantially higher. Of potential interest is the possible dropout of XUV-disk galaxies in systems having globally averaged sSFR less than $2 \times$ $10^{-10} \mathrm{yr}^{-1}$, marked with a dashed line in the diagram. Of the 26 sample galaxies below this limit in the sample, only $3(11 \%)$ are XUV-disk detections. It does not seem that this is a detection threshold issue, as most XUV-disks are identified on the basis of clumps that are individually significant, even if sparsely distributed in the outer disk. Two ways to support continued star formation of any type (besides closed-box recycling, e.g., Boselli et al. 2001) are accretion from the IGM and gas deposition resulting from the debris of galaxy interaction processes. It could be that the very low sSFR galaxies are those that have started to exhaust their (internal) gas supply and are not being augmented through such external means. If this is true, then the mechanisms to supply the outer disk with gas (Beckman et al. 2004; Kereš et al. 2005; Bournaud et al. 2005) would also be missing, leading to the observed lack of XUV-disk structures.

The gaseous content of the galaxies in our sample is well characterized ( $\$ 2.1$ ). In Figure $9 b$ we show the distribution of $M_{\mathrm{H}} / L_{B}$ versus $L_{K}$ for XUV-disks in comparison to ordinary galaxies. The $M_{\mathrm{H}_{\mathrm{I}}} / L_{B}$ ratio is commonly utilized to classify gas-rich and gas-poor systems. XUV-disks of all type (but especially Type 1) are systematically displaced from the overall distribution in the sense that they are gas-rich (by about a factor of 2 from the survey median at constant $L_{K}$ ). This enhancement is
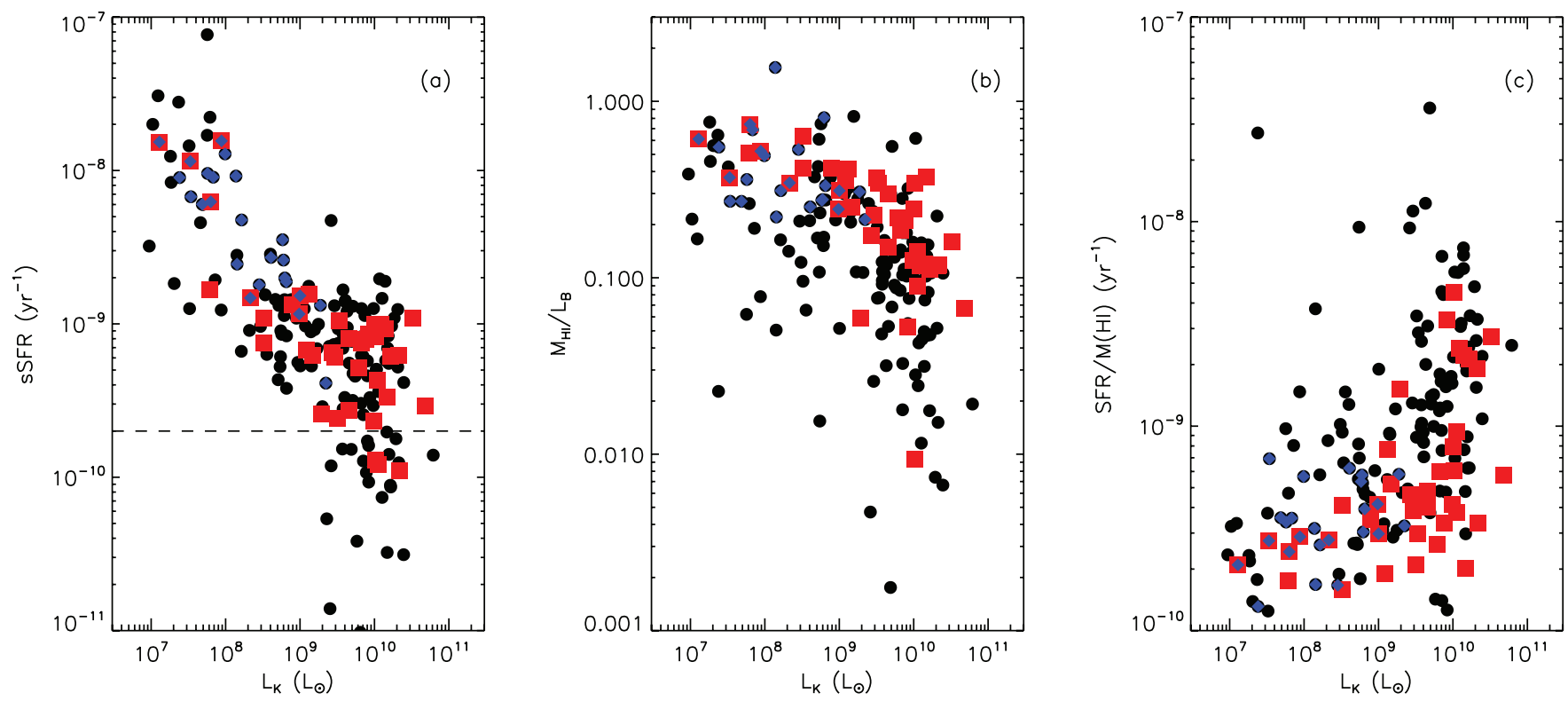

FIG. 9.- (a) Distribution of globally averaged specific SFR (sSFR) vs. $L_{K}$ for the survey sample (black circles) and for galaxies classified as XUV-disks (red squares,

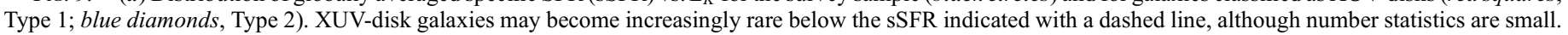
(b) Distribution of globally averaged $M(\mathrm{HI}) / L_{B}$ vs. $L_{K}$. (c) Distribution of globally averaged SFR $/ M(\mathrm{HI})$, a measure of star formation efficiency, vs. $L_{K}$. 

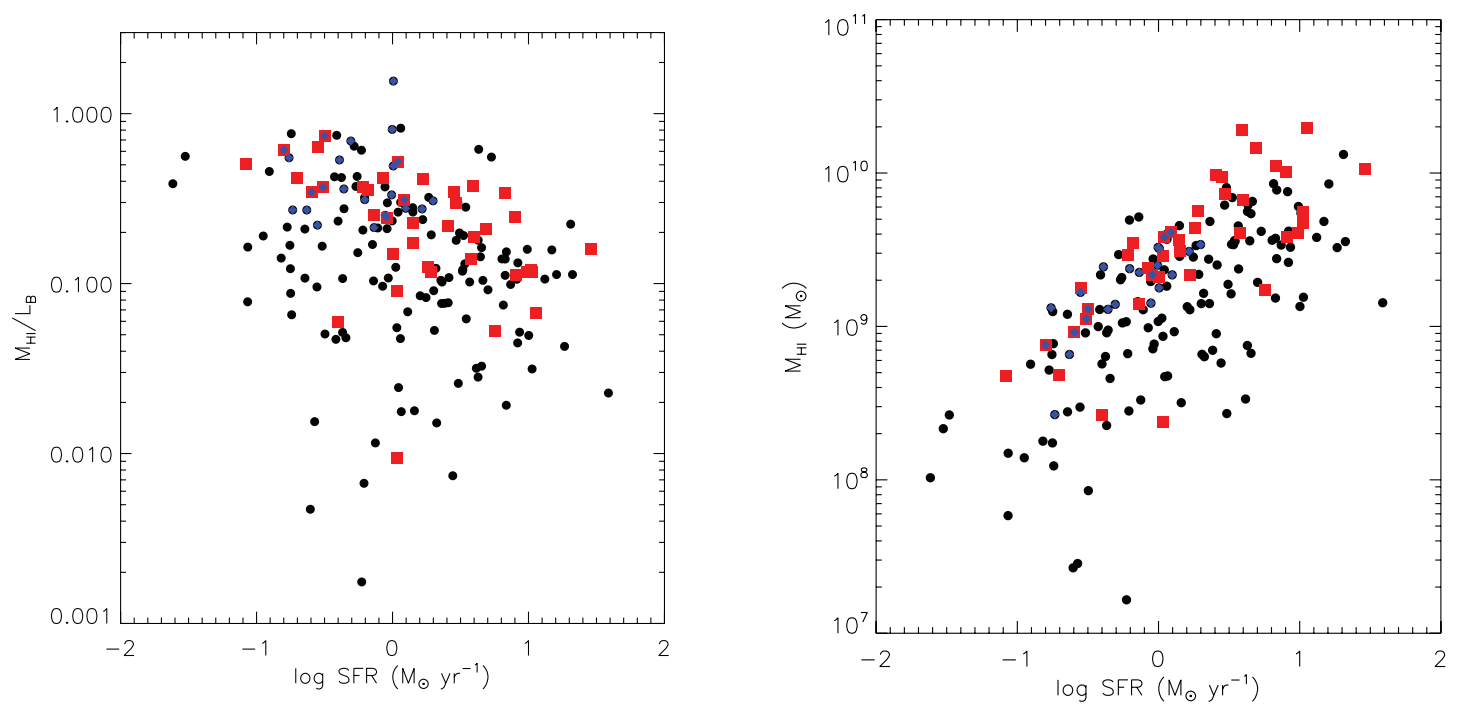

FIG. 10.- (a) Distribution of globally averaged $M\left(\mathrm{H}_{\mathrm{I}}\right) / L_{B}$ vs. SFR for the survey sample (black circles) and for galaxies classified as XUV-disks (red squares, Type 1 ; blue diamonds, Type 2). (b) Distribution of $M(\mathrm{H} \mathrm{I})$ vs. SFR.

what one would expect for the Type 1 XUV-disks; if the outer disk gas is not present, we would not see UV evidence for star formation unless the $\mathrm{H}$ i was very recently stripped (as might happen in a cluster environment; e.g., Boselli et al. 2006; Crowl et al. 2006; Crowl \& Kenney 2006; Vollmer et al. 2006). Although the increased gas-richness in XUV-disks is not surprising, our knowledge is sparse concerning the origin of the outer disk gas and the mechanisms promoting SF therein. It will be particularly informative to study galaxies having extended $\mathrm{H}$ I with and without XUV-disk structure, looking for changes in their kinematics, morphology, and cool/warm neutral medium (CNM/WNM) phase balance.

Figure $9 c$ presents a measure of global star formation efficiency $\left(\mathrm{SFE}=\mathrm{SFR} / M_{\mathrm{H}}\right)$ versus $L_{K}$. Type $1 \mathrm{XUV}$-disks tend to occupy lower SFR $/ M_{\mathrm{H}}$ than the median for the sample at all $L_{K}$, whereas Type 2 objects are typical of the entire sample at low $L_{K}$. In Type $1 \mathrm{XUV}$-disks, the average SFE in the outer disk is certainly low (driving the global value down), but probably still reaches typical levels (for within an ordinary disk) on localized scales in $\mathrm{H}$ I arms and/or clumps. Apparent gas consumption timescales (1/SFE) are significant (several Gyr) for most of the XUV-disks, although the issue is complicated due to star-to-gas recycling and the possibility of $\mathrm{H}$ I production via $\mathrm{H}_{2}$ photodissociation, as discussed by Gil de Paz et al. (2007b) and originally by Allen et al. (1986).

Figure 9 suggests that XUV-disk galaxies are generally gasrich for their stellar mass and that the SF activity which makes them remarkable is occurring in a low SFE mode. In Figure 10, we examine whether XUV-disk galaxies are more gas-rich than other galaxies of similar global SFR, given the correlation of gas mass with SFR. Figure $10 a$ shows $M_{\mathrm{H}} / L_{B}$ versus SFR, and Figure $10 b$ presents $M_{\mathrm{H}_{\mathrm{I}}}$ versus SFR. We conclude that (with and without normalization by $L_{B}$ ) XUV-disk galaxies do have enhanced $\mathrm{H}$ I content as a class, relative to spirals of matching global SFR.

Galactic star formation activity is not disclosed completely by emission in either the IR or UV bands (except in exceptional cases, e.g., ULIRGs or UVLGs), but rather by the bolometric energy output of young stars. It has been shown that the relative contributions of TIR and UV systematically vary as a function of Hubble type (Gil de Paz et al. 2007a; Dale et al. 2007) and
NIR luminosity (Cortese et al. 2006), but with dispersion approaching $\sim 0.5-1.0 \mathrm{dex}$ in TIR/UV. What is the origin of such scatter, far in excess of observation errors? One possibility is a mix of internal environments, and the relative balance (by recent SFR) of these locales. Both varieties of XUV-disks possess a region in which the bolometric output is overwhelmingly dominated by UV luminosity in deference to the IR. Figure 11 shows that XUV-disks tend to have a relatively low TIR/UV ratio, compared to other galaxies of the same $T$-type and stellar mass (traced by $L_{K}$ ), preferentially near the bottom locus of the overall distribution. In Type 2 disks this difference in TIR/UV may be wholly explained by the UVemission coming from the LSB zone (which has relatively low dust content and low metal abundance). For Type 1 XUV-disks, the observed offset to bluer TIR/UV (by several
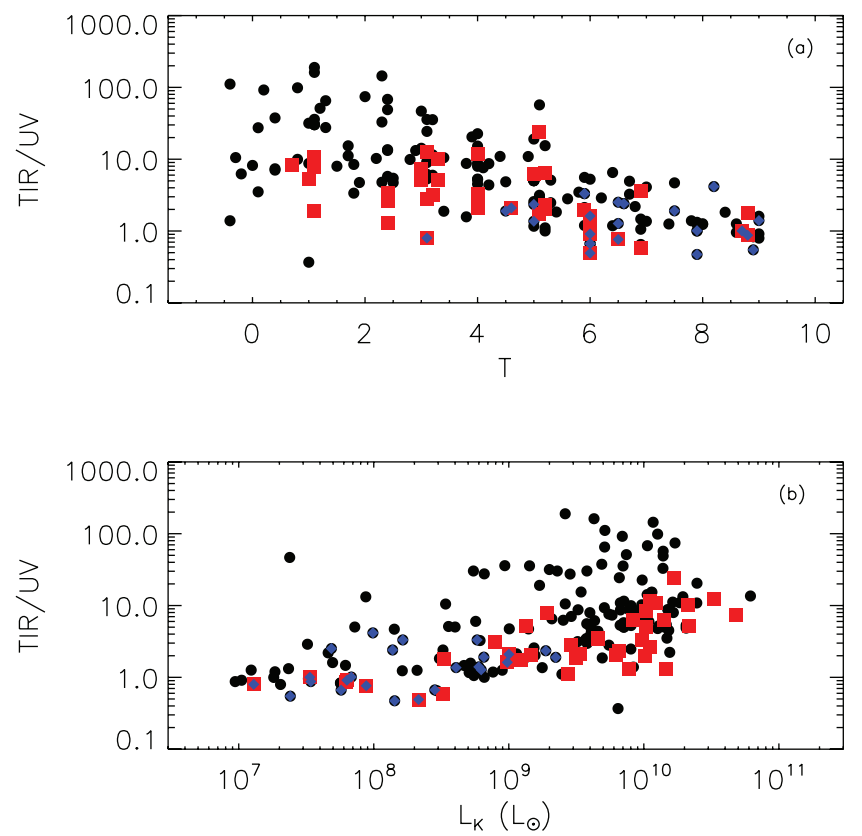

FIG. 11.-(a) Distribution of globally averaged TIR/UV vs. $T$ for the survey sample (black circles) and for galaxies classified as XUV-disks (red squares, Type 1; blue diamonds, Type 2). (b) Distribution of globally averaged TIR/UV vs. $L_{K}$. 


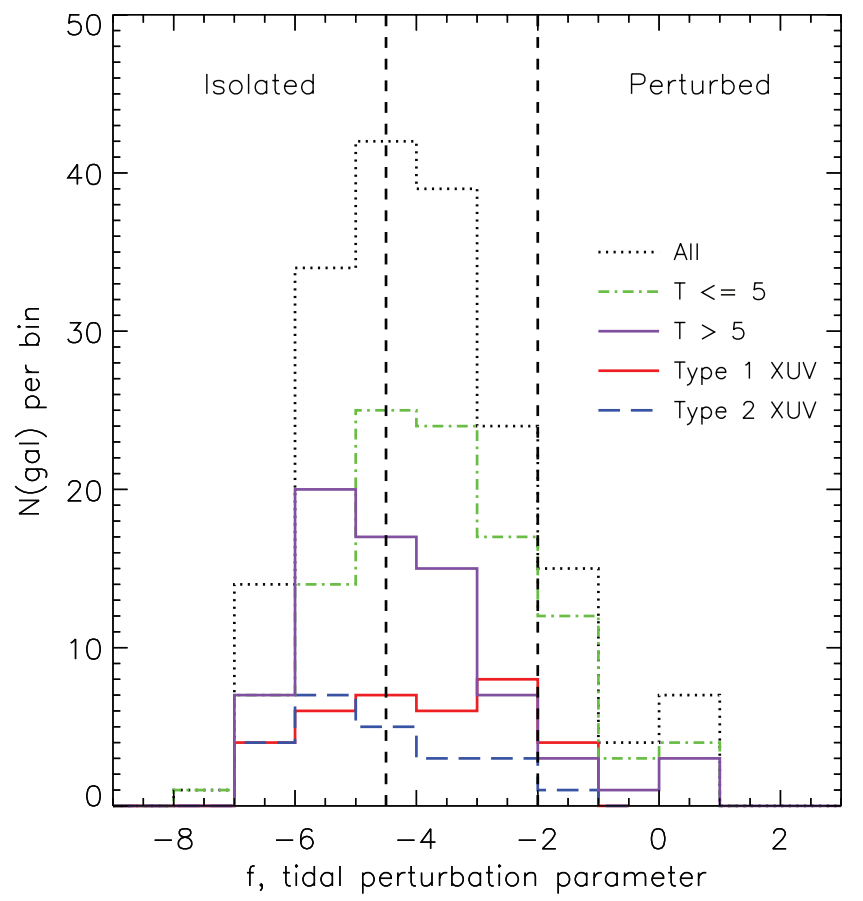

FIG. 12.-Tidal perturbation parameter, $f$, for all galaxies in our current survey (black dotted line) compared to those classified as XUV-disks (red solid line, Type 1 ; blue dashed line, Type 2). We also plot the sample-wide distribution of $f$ for early- and late-type spirals with green dash-dot and purple dash-triple-dot lines, respectively. The designation of isolated and perturbed labels follows the limits proposed by Varela et al. (2004).

tenths of a dex) is likely due to more than just the contribution of UV emission from the outer disk, which is very small percentage of total UV luminosity.

\subsubsection{Evidence for Interaction?}

Some XUV-disk galaxies described in Appendix A are clearly interacting galaxies, have obvious nearby companions, or show morphological clues suggestive of external perturbation (optically, or in the UV). This should not be surprising, given the fact that $60 \%$ of galaxies belong to a pair or group of massive galaxies (Giuricin et al. 2000), and a higher percentage must have at least some low-luminosity companion. Nevertheless, rather isolated galaxies do exist (Galletta et al. 2006; Varela et al. 2004; Pisano \& Wilcots 1999, 2003), even in the XUV-disk population. The situation is complicated further because the mere fact that a galaxy has companions does not imply that they have actually had any influence on the recent star formation activity (100+ Myr timescales). Boselli \& Gavazzi (2006) review the important role of environmental effects on galaxy evolution. Note that in addition to the stimulation of SF, external perturbation can remove outer disk gas, stopping $\mathrm{SF}$ and producing truncated UV disks (e.g., NGC 4569; Boselli et al. 2006).

In order to quantify the potential effectiveness of interactioninduced gas motions as a driver of outer disk SF, we computed the tidal perturbation parameter, $f\left(Q_{D}\right.$ in Dahari 1984; Salo 1991; Byrd \& Howard 1992), with our calculation following Varela et al. (2004). This measure specifies the ratio of the tidal force imposed on a galaxy $(G)$ of size $R$ by a possible perturber $(P)$ and the internal force per unit mass in the outer part of galaxy $G$. Varela et al. (2004) demonstrated that $f$ can be successfully computed using relative apparent magnitudes as a proxy for the mass ratio of galaxies $P$ and $G$, as called for in the seminal papers referenced above, and the projected separation rather than

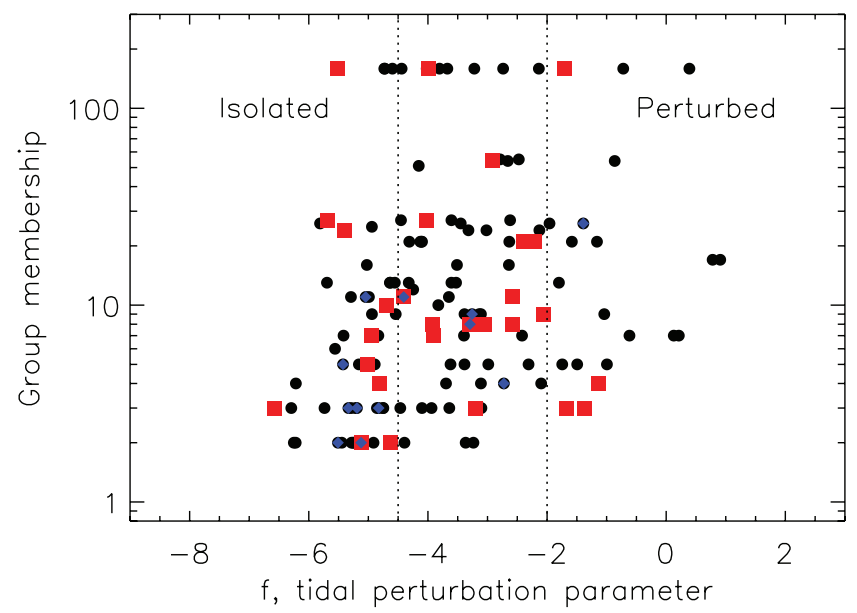

FIG. 13.-Comparison of NOG group membership vs. $f$, the tidal perturbation parameter. Note that a fraction of our sample galaxies (black circles) and of the XUV disk subset (red squares, Type 1; blue diamonds, Type 2) are not represented in this panel either because they were not in a group/pair or because they are too faint to have even been considered in the NOG grouping analysis of Giuricin et al. (2000).

a true three-dimensional impact (pericenter) parameter, $b$ (Icke 1985). For all galaxies in our sample, we computed the value of $f$ appropriate for each possible perturber culled from the LEDA galaxy catalog. Note that the LEDA catalog is not homogeneous. In this sense, the computed $f$ might depend strongly on whether or not SDSS catalogs with coverage for any galaxy of interest have been incorporated into LEDA. We eliminated clearly unassociated galaxies for each primary in the same manner as Varela, using checks on the Virgo-corrected relative radial velocity $\left(V_{\text {vir }}\right)$, spatial size $(D \geq 2 \mathrm{kpc})$, and absolute magnitude $(B \leq-12)$. We record the maximum value of such an array of $f$-values for each sample galaxy, and interpret this as an indicator of strength for the dominant interaction. Varela et al. (2004) denoted galaxies with $f<-4.5$ to be "isolated" or, more precisely, tidally unaffected in the recent past. They also defined galaxies with $f>-2.0$ to be "perturbed." In Figure 12 we show the distribution of $f$ for all the galaxies in our sample and for the two classes of XUV-disk galaxy. We find that XUV-disks with Type 1 structures have a relatively flat $f$ distribution, despite the strongly peaked $f$ distribution (centered near $f=-4.5$ ) for the entire galaxy sample. However, a K-S test shows that this difference is probably not significantthe Type $1 f$ distribution agrees with the entire sample at $91 \%$ confidence. If Type 1 structures are linked to external perturbation, it is of a level commonly encountered in the spiral galaxy population, and not of the strength formally defined as perturbed by Varela et al. (2004). On the other hand, the Type 2 XUV disks are slightly more isolated than not (only $29 \%$ chance agreement with entire sample), although a wide range in $f$ is still seen for these galaxies. We note that the $f$-distribution of Type 2 disks matches that of late-type spirals ( $91 \%$ confidence), so the isolation may simply be a reflection of the progenitor galaxy population.

The tidal perturbation parameter is not the only way of quantifying a galaxy's environment, and indeed has some limitations. Note that the tidal perturbation parameter would not classify a merger remnant as perturbed (since each merging object would no longer be distinct, e.g., XUV-disk NGC 2782). Also, a galaxy may live in a high-density location such as a group, without a current perturbation. For instance, NGC 3198 is the brightest galaxy in a group offive objects, but is labeled isolated according to the tidal perturbation parameter. In the framework of hierarchical galaxy assembly, one might expect that the frequency of 
galaxy interactions leading to accretion would scale with the number of neighbors constituting a group, or even more so with group density. Figure 13 shows the distribution of $f$ versus the number of galaxies in a NOGG group. We find the most isolated galaxies become increasingly rare in richer groups. Second, perturbed galaxies occur in groups of any size. XUV-disks can be found in galaxy groups both rich and poor. In fact, some of the dynamically isolated XUV disks shown in Figure 12 are actually located in groups containing 10 or more galaxies (e.g., NGC 4254 in the Virgo Cluster and NGC 4414 in the Coma I group).

Finally, a galaxy could be perturbed by undetected low-mass/ low surface brightness objects in their vicinity, or even highvelocity clouds with gas but no stellar component. Deep, dedicated survey observations to disclose any such perturbers are underway, but at present we looked for morphological clues suggestive of interaction. We have noted (in Table 2) those XUV disk galaxies which have peculiarities at UV/optical wavelengths, or which are known through the literature to be interacting in some manner. Although based on heterogeneous data and analyses, more than $75 \%$ of the Type 1 XUV-disk objects show morphological or $\mathrm{H}$ I evidence for interaction/merger or minor external perturbation of some sort (e.g., HVCs). About half of the Type 2 XUV-disks appear to be recently disturbed. In at least some of these cases, the tidal perturbation parameter falsely indicates that they are isolated. Note that we did not scrutinize non-XUV galaxies in our sample at the same level of detail, as this is beyond the scope of our paper. For this reason, the possible importance of interaction/merger or external perturbation in XUV-disks must remain suggestive only.

\section{DISCUSSION}

\subsection{Efficacy of SF Tracers at Low SFR}

Our GALEX observations demonstrate that the domain of star formation activity in some spiral galaxies has been underestimated, both spatially and in terms of azimuthally averaged gas column density. Note that outer disk star formation was previously detected in a few galaxies (e.g., Ferguson et al. 1998b; Ferguson 1998) using deep $\mathrm{H} \alpha$ images, which make it possible to detect a single O star (see Gil de Paz et al. 2007b for M83) at distances well beyond the Local volume. However, GALEX shows that such SF is relatively commonplace.

As shown by Boissier et al. (2007), $\mathrm{H} \alpha$ observations of the $\mathrm{H}$ II region population become unreliable in regions of very low $\Sigma_{\mathrm{SFR}}$, merely due to the short timescale $\left(t_{\mathrm{H} \text { II }}\right)$ over which any one $\mathrm{H}_{\text {II }}$ region remains visible. As $\Sigma_{\mathrm{SFR}}$ decreases, there comes a point at which the probability of an $\mathrm{H}$ II region forming during the preceding $t_{\mathrm{H}}$ in the (finite) area of interest drops substantially below unity. In contrast, UV-emitting B stars survive more than an order of magnitude longer than the $\mathrm{O}$ stars responsible for ionization, increasing the odds of detecting rare, but still present, star-forming regions. Our UV observations, and such an interpretation, are consistent with the limited population of outer disk $\mathrm{H}$ II regions known in a few galaxies (Ferguson et al. 1998b; Ryan-Weber et al. 2004; Gil de Paz et al. 2007b). Moreover, we emphasize that the nondetection of line emission from any given object (even with good $\mathrm{H} \alpha$ sensitivity) should not be taken as evidence that quasi-steady-state $\mathrm{SF}$ is ruled out at very low $\Sigma_{\mathrm{SFR}}$. Even UV imaging has a similar limitation (although at much lower $\Sigma_{\text {SFR }}$ ), beyond which only color-magnitude diagram (CMD) analysis for resolved stellar populations can effectively recover knowledge of past SF activity.

The size of the particular region being surveyed has an impact on practical limits for the SF tracers because it is the integrated
SFR which ultimately drives the counting statistics of $\mathrm{H}$ II regions and older (non-H $\alpha$-emitting) clusters. In the low-SFR regime, each tracer eventually suffers from stochastic incompleteness and uncertainty, which cannot be overcome by more sensitive observations.

We have conducted simple Monte Carlo simulations to model the visibility of a continuously star-forming population with UV and $\mathrm{H} \alpha$ tracers, subject to the size-of-sample selection effect described above. We assumed a cluster formation rate (CFR, clusters $\mathrm{Myr}^{-1}$ ), cluster membership function (CMF), and a Salpeter IMF. See Thilker et al. (2002) for details of the modeling procedure. The number of stars per cluster was allowed to reach down to 1 . We tabulated the number of clusters having one or more ionizing $\mathrm{O}$ star and the number of clusters with surviving OB stars. These metrics were evaluated for many independent snapshots throughout each model run.

In Figure 14, we plot the probability distribution function and cumulative probability distribution function for the expected number of clusters still containing $\mathrm{O}$ stars, given continuous $\mathrm{SF}$ with average $\log$ (SFR) equal to $-4.5,-4.0,-3.5$, and $-3.0\left(M_{\odot} \mathrm{yr}^{-1}\right)$. The assumed CMF can have an effect on the predicted population of O star hosting clusters. Figures $14 a-14 h$ present two models for each SFR, differing only in the treatment of the upper CMF. The slope of the CMF in both cases was set to -2 . However, it is plausible that the most massive/populated clusters may be inhibited from forming in the outer disk due to a lack of massive cool clouds. Braun (1997) showed that the CNM disappears rapidly beyond the $D_{25}$ radius, leaving $\mathrm{H}$ I predominantly in the form of WNM. Therefore, we conducted alternative model runs with the cluster membership function truncated such that the maximum cluster mass is $10^{3} M_{\odot}$. The default models have a CMF allowing up to $10^{6} M_{\odot}$ as the maximum cluster mass. In Figure 14, the default models are shown in the left column (Figs. 14a-14d) and the truncated CMF models are shown in the center column (Figs. 14e-14h). We note that for the default model in low SFR environments (Fig. 14d), such as XUVdisks, the probability of not finding $\mathrm{O}$ star hosting clusters in the region being studied reaches as high as $80 \%$, despite ongoing star formation. Truncating the CMF has the effect of increasing the typical predicted $\mathrm{H}$ II region population, because less mass is locked up in massive clusters, which in turn allows for the more frequent creation of typical clusters.

The assumed IMF has a more pronounced effect on the expected population of $\mathrm{O}$ stars at any given time in a low SFR environment, especially if the maximum stellar mass is a function of cluster mass. Some theoretical and observational studies support such an effect (Bonnell 2000), but the idea is still under debate. For instance, Elmegreen (2006) reported that the average maximum stellar mass in a cluster population decreases with decreasing cluster mass, but that the absolute maximum stellar mass is roughly constant. In Figures $14 i-14 l$, we show plots equivalent to those in Figures $14 e-14 h$ (restricted CMF), except that we imposed a lower limit of 100 stars with $M>M_{\odot}$ for the initial cluster membership necessary to produce stars with $M \geq 10 M_{\odot}$. Figure $14 i$ shows that even for integrated SFR large as $1 \times 10^{-3} M_{\odot} \mathrm{yr}^{-1}$, approximately $40 \%$ of the time a region of interest (e.g., sized $1 \mathrm{kpc}^{2}$ for $\Sigma_{\mathrm{SFR}}=1 \times 10^{-3}$, $10 \mathrm{kpc}^{2}$ for $\Sigma_{\mathrm{SFR}}=1 \times 10^{-4}$ ) will lack an O star hosting cluster (and associated H II emission). At lower SFRs (Figs. 14j-14l), the probability is $70 \%-95 \%$.

The simple simulations presented here represent the best-case scenario for detection of $\mathrm{H}$ II regions in low $\Sigma_{\text {SFR }}$ environments, as our current model has no allowance for extinction, gas density effects, or variation in overall population age [via the SFH, hence 

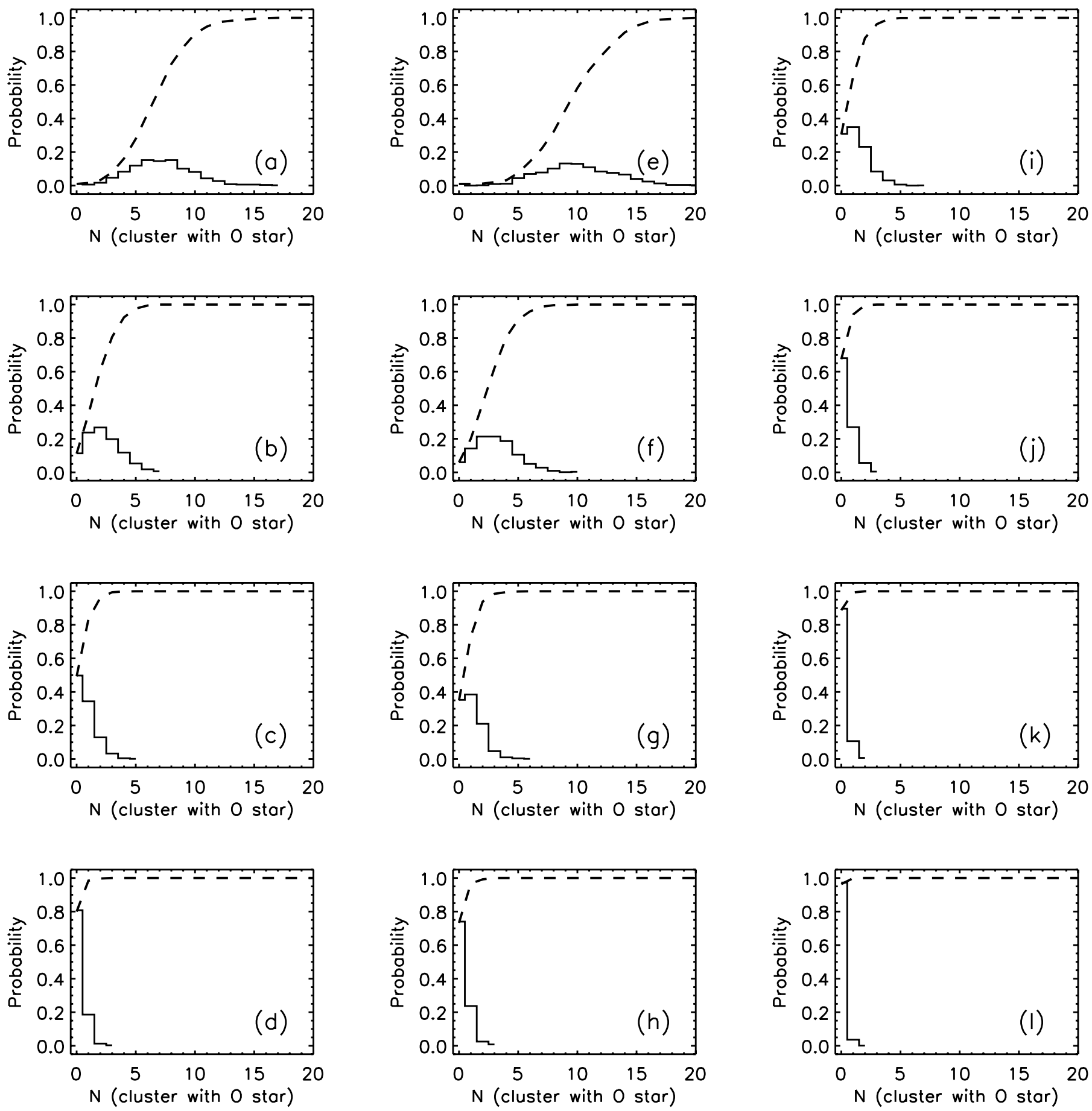

Fig. 14. - Probability of $N$ clusters each containing at least one surviving $\mathrm{O}$ star for various Monte Carlo models. Probability distribution functions (solid histogram) and cumulative distributions (dashed line) are plotted. The simulated $\log (\mathrm{SFR})$ value is $-3.0,-3.5,-4.0$, and $-4.5 M_{\odot} \mathrm{yr}^{-1}$ from the top row to bottom row, with SF distributed throughout a region of arbitrary size. We assume a Salpeter IMF for the left and middle columns (panels $a-h$ ), and a conditionally truncated Salpeter IMF for the right column (panels $i-l$ ). In the later case, we assumed massive stars $\left(M \geq 10 M_{\odot}\right.$ ) may only form in clusters having initial membership of at least 100 stars with $M>1 M_{\odot}$. The left column has a default CMF of slope -2 , allowing clusters up to $10^{6} M_{\odot}$, whereas the middle and right columns have an upper limit to the $\mathrm{CMF}$ at $10^{3} M_{\odot}$.

$\mathrm{CFR}(t)]$. Furthermore, the simulations do not account for observational sensitivity. In reality, (younger) clusters still hosting at least a single $\mathrm{O}$ star probably have nonnegligible extinction, and would certainly be subject to systematically more attenuation than old clusters, which eventually disperse from the dust associated with their birthplace. Initial estimates of extinction toward line-emitting regions in the XUV-disks of M83 and NGC 4625 show that values of $E(B-V)$ at the level of a few tenths of a magnitude are common (Gil de Paz et al. 2007b). The outer disk
ISM is substantially more tenuous than typical environments at smaller galactocentric distances. Such low density can have the deleterious effect of dimming the surface brightness of $\mathrm{H} \alpha$ emission associated with $\mathrm{H}$ II regions (Elmegreen \& Hunter 2006), and in principle could encourage leakage of Lyman continuum photons for porous or otherwise density-bounded clouds. However, it is unclear how much these effects will be actualized, given that the SF occurs within local overdensities, even if the general outer disk environment is of quite low average density. 

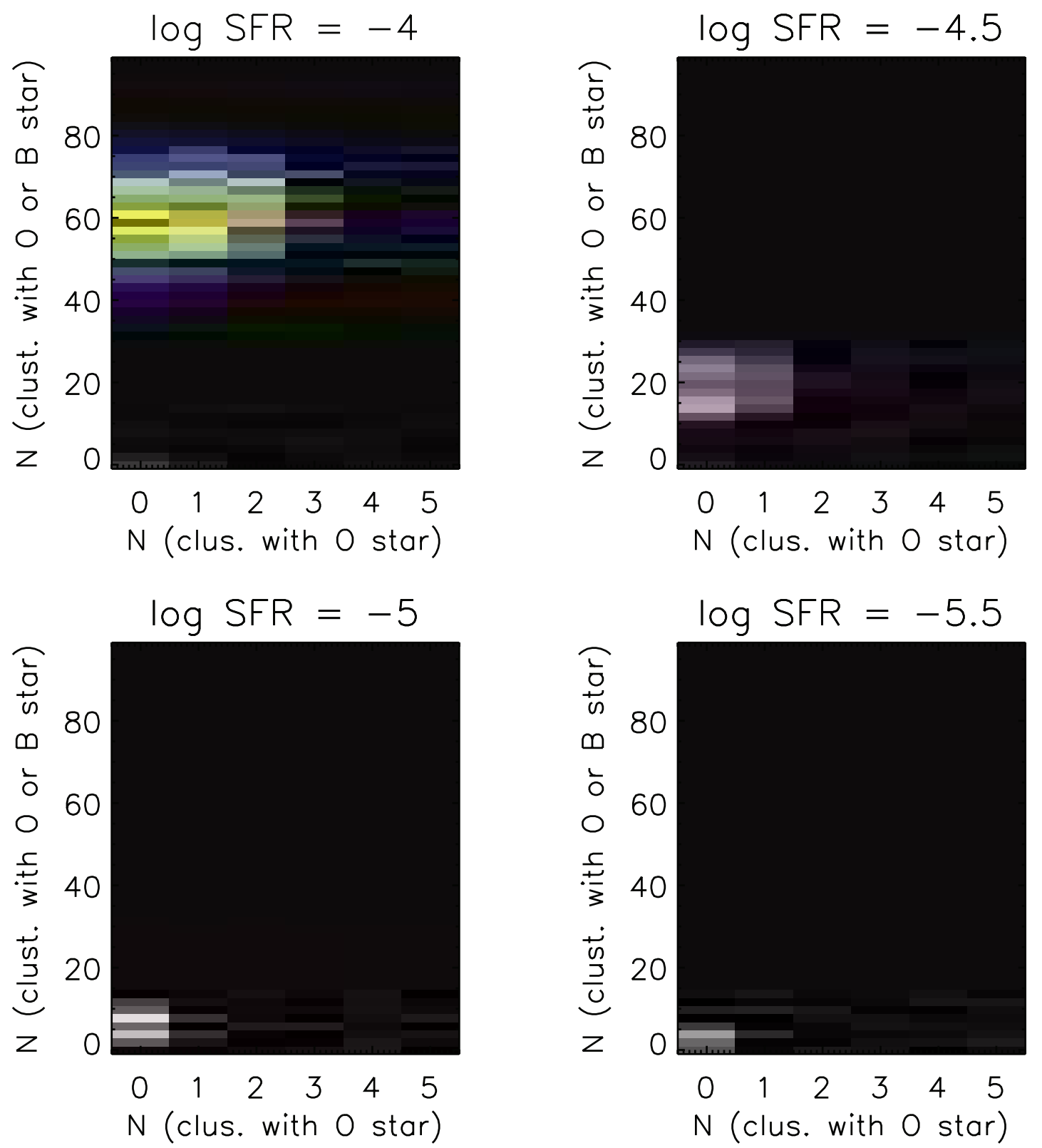

FIG. 15.-Predicted number of clusters containing at least one $\mathrm{O}$ star and the number of clusters containing one or more $\mathrm{OB}$ stars, for $\log (\mathrm{SFR})=-4.0,-4.5,-5.0$, and $-5.5 M_{\odot} \mathrm{yr}^{-1}$ integrated over an arbitrary region of interest. The probability distribution function shows that the UV SFR tracer (sensitive to OB stars) is stochastically limited for $\log (\mathrm{SFR}) \leq-5.5$.

Gil de Paz et al. (2007b) demonstrate that the few $\mathrm{H}$ II regions found in the XUV-disks of M83 and NGC 4625 are compact, in keeping with this notion of localized SF. Our current Monte Carlo runs assumed a constant SFR, although cluster formation times are randomized. Clearly, models computed for the period following a burstlike SFH (whether instantaneous, Gaussian, or declining over time) will tend to exacerbate the bias against detecting $\mathrm{H}$ II regions in the outer disk illustrated by our current models and Boissier et al. (2007). Elmegreen \& Scalo (2006) showed that the (apparent) present-day mass function (PDMF) is influenced by SFH.

Our Monte Carlo models also allow tracking of the number of clusters containing less massive, but nevertheless UV-emitting, B stars. As we noted above, even UV emission may eventually falter as a SFR tracer at quite low SFRs (when the typical probability of having a cluster containing one or more B star drops below unity). Figure 15 shows the bivariate PDF representing the predicted number of clusters containing at least one $\mathrm{O}$ star and the number of clusters containing one or more OB stars. For moderate to large SFRs (not shown), the $N(\mathrm{OB}) / N(\mathrm{O})$ ratio is effectively constant for the continuous SF scenario. At low SFRs, $N(\mathrm{O})$ becomes stochastically limited first [near log (SFR) = $-3.5]$, followed by $N(\mathrm{OB})$, which starts showing a significant fraction of "misses" by $\log (\mathrm{SFR}) \sim-5.5$. Does the universe have any environments for which this stochastic limit to the UV-emitting cluster population is relevant? Adopting the Schmidt law parameterization of Boissier et al. (2007), the form of which employs total $\left(\mathrm{H} \mathrm{I}+\mathrm{H}_{2}\right)$ gas surface density, we find that $\Sigma_{\left(\mathrm{H}_{\mathrm{I}}+\mathrm{H}_{2}\right)}=0.1 M_{\odot} \mathrm{pc}^{-2}$ corresponds to a predicted $\log \left(\Sigma_{\mathrm{SFR}}\right)=$ $-6.0\left(M_{\odot} \mathrm{yr}^{-1} \mathrm{kpc}^{-2}\right)$. Obviously, this prediction neglects the possibility of a star formation threshold. Such values of $\Sigma_{\left(\mathrm{H}_{\mathrm{I}}+\mathrm{H}_{2}\right)}$ and $\Sigma_{\text {SFR }}$ are a factor of several below the observed limit of each variable in all studies to our knowledge, even in the XUV-disk environment. Thus, it would seem (at face value) that the stochastic 
limit of the UV SFR tracer is most likely never encountered in the overall galaxy population. It is remotely conceivable although that this could be a selection effect, associated with the fact that $\mathrm{H}$ I observations of nearby galaxies almost never probe such low gas surface densities (one exception is M31; see Braun $\&$ Thilker 2004). We know that low $N_{\mathrm{H}_{\mathrm{I}}}$ gas does surround galaxy disks, from QSO absorption line measurements and directly in emission, as demonstrated by Figure 12 of Braun \& Thilker. Although we do not consider it likely, it is worth mentioning that extremely isolated cool clouds could form quasi-continuously in the typically warm and diffuse low column density halo gas, and subsequently be missed (as H II regions are) due to their short lifetime. In this case, the stochastic limit of UV SFR measurement could indeed be relevant.

Radial surface brightness profiles for the first-known XUVdisks, M83 and NGC 4625, originally raised the issue of potential discrepancy between $\mathrm{H} \alpha$ and UV emission. With hindsight, the relative dropout of the $\mathrm{H} \alpha$ profile may not have signaled a fundamental change in the star formation properties between inner and outer disk, but rather brought about a new view regarding the efficacy of tracers for the young-to-moderate age populations that do exist, and for the remarkable persistence of star formation in any environment which is locally populated by a cool ISM component. Above we noted reasons for the lack of detection of $\mathrm{H}$ II regions, or for suppression of their formation, but we emphasize that $\mathrm{H}$ II regions are sometimes present (albeit rarely) in the XUV-disk environment and can be observed if one images deeply enough (e.g., NGC 0628, Ferguson et al. 1998b, Ferguson 1998; M83 and NGC 4625, Gil de Paz et al. 2007b).

As noted in the description for a few XUV-disk galaxies studied in this paper, we detect UV emission from young stars in portions of the galaxy not even reached by traditional surface brightness profile analysis (based on the same observations, with profile extent conservatively set by error limits). Such sources are located in regions of the XUV-disk having azimuthally averaged $\mu(\mathrm{FUV})>30 \mathrm{mag} \operatorname{arcsec}^{-2}$. At such low $\Sigma_{\mathrm{SFR}}$, star-forming clusters are often difficult to distinguish from the population of background galaxies, except if there are clusters clumped into a larger structure. This demonstrates that, even with GALEX, it will remain difficult to confidently determine the maximum spatial extent of star formation in disk galaxies. A combination of GALEX imaging and higher resolution follow-up is required for this purpose. $\mathrm{H}$ I maps can also be used for guidance as to which extended locations are most likely to host SF. These considerations suggest that in order to achieve a robust surface brightness profile in the outermost portions of a galaxy having sparsely distributed clusters, one must resort to masking of contaminating sources via inspection. However, we emphasize that for outer radii (with a negligible source covering factor) obtaining an azimuthally averaged profile is nearly a moot point, as it is no longer an appropriate description of the environment. Rather, the SF characteristics of outer disk, low $-\Sigma_{\mathrm{SFR}}$ locations would be better described in a probabilistic manner based on the observed PDF for UV emission as a function of variables such as local $\Sigma_{\text {gas }}, \Sigma_{\text {stars }}$, and dynamical quantities.

\subsection{SF Thresholds and Disk Instability}

Given the discussion above concerning SFR tracers in the XUV-disk environment, how much evidence remains to support the much-debated SF threshold in all its incarnations (e.g., azimuthally averaged vs. local, dynamically variant vs. fixed critical column density)?

At the very least, XUV-disks highlight the long-recognized but often overlooked fact that the Toomre criterion for gravi- tational stability, although derived in a one-dimensional manner (radially), should not be interpreted in the same sense. It does not allow one to delineate an outer zone devoid of all SF. GALEX shows extensive $\mathrm{SF}$ in subcritical zones for which $Q>1$. In fact, Kennicutt (1989) and Martin \& Kennicutt (2001) concluded early that localized peaks in the gas distribution at otherwise subcritical radii can be gravitationally unstable and form stars. In their work on NGC 6822, de Blok \& Walter (2006) noted that an inherently two-dimensional version of the Toomre criterion would be ideal, but can only be partially attained given that the disk rotation velocity cannot be determined on a pointby-point basis for position angles substantially removed from a galaxy's major axis. Nevertheless, de Blok \& Walter showed the improvement in identifying SF-susceptible sites achieved just by comparing the local $\Sigma_{\text {gas }}$ to an azimuthally averaged $\Sigma_{\text {crit }}$, from the traditional Toomre formalism. To summarize, it is not surprising that SF persists in outer clumps of gas having local $\Sigma_{\text {gas }} \geq$ $\Sigma_{\text {crit }}$ embedded in an otherwise subcritical environment.

The detailed ISM structure in the outer disk zone is fundamentally what needs to be considered when judging if SF is likely or unlikely. Elmegreen \& Parravano (1994) first suggested that the local phase balance of the ISM is directly tied to the presence or absence of star formation. Specifically, they showed that a two-phase $\mathrm{H}$ I medium (allowed under conditions of sufficient thermal pressure) which contains a cool component is a prerequisite to SF. This view has been recently revived by the modeling of Schaye (2004), who derived a local column density required for the formation of CNM. The precipitous decline of the fractional amount of $\mathrm{H}$ I contained in the cool phase at the edge of the optical disk ( $\sim D_{25}$ radius) was first reported observationally by Braun (1997). The notion that local conditions are most crucial in determining the observed $\Sigma_{\text {SFR }}$ meshes very well with our GALEX observations.

How then should one best model the anticipated radial distribution of star formation rate, given the apparent importance of local conditions? Elmegreen \& Hunter (2006) presented a multicomponent model of star formation in disk galaxies which included not only fundamentally gravitational (Toomre) instability, but also an allowance for secondary pathways to cloud collapse, such as spiral wave shocks, turbulence compression, and localized triggering - all of which can persist into the outer disk. They assumed a lognormal $\Sigma_{\text {gas }}$ distribution matched to the average $\Sigma_{\text {gas }}$ at each radius, which naturally allows for a limited fraction of high-density regions even at subcritical average $\Sigma_{\text {gas }}$. Such a lognormal distribution is appropriate for turbulent media. The Toomre criterion was then applied in a local manner to modeled gas surface densities, to see if the sites of secondary cloud formation became gravitationally unstable. A standard Schmidt law parameterization of the SFR density appropriate for unstable clouds was assumed thereafter. The Elmegreen \& Hunter (2006) models appear largely capable of reproducing diverse SFR profiles such as those seen by GALEX for XUV-disks and by Hunter \& Elmegreen (2006) for irregular galaxies.

Even if GALEX UV and earlier $\mathrm{H} \alpha$ observations (e.g., Ferguson et al. 1998b) together show that SF frequently occurs well beyond the $D_{25}$ radius and into subcritical radii, we suggest that the Toomre criterion does rather accurately predict the galactocentric distance at which ISM properties change from an $\mathrm{H}$ I distribution effectively dominated by massive cool clouds to one in which the warm diffuse medium becomes prominent and, as such, has an impact on the SFR (UV and $\mathrm{H} \alpha$ ) profiles. Braun (1997) showed that this CNM/WNM transition is also associated with a drop in $\mathrm{H}$ I mass surface density. The models of Elmegreen 
\& Hunter (2006) already indicated that breaks in the SFR profile can be linked to the general loss of Toomre's gravitationally unstable clouds, but they assumed one of two (conservative) smoothly varying gas profiles (pure exponential, or exponential with transition to $1 / R$ at large galactocentric distances). In reality, the $\mathrm{H}_{\mathrm{I}}$ column density often drops faster than this for a limited range in galactocentric distance near the $D_{25}$ radius, leading to a jump/ discontinuity in SFR density profiles. This true discontinuity worked in concert with the stochastic, size-of-sample effect and other factors mentioned in $\S 4.1$ to give the appearance of a genuine SF threshold (e.g., Martin \& Kennicutt 2001).

In summary, with GALEX we are beginning to probe outer disk environments with average $\Sigma_{\text {gas }}$ low enough (e.g., at $\Sigma_{\text {gas }} \leq$ $0.1 M_{\odot} \mathrm{pc}^{-2}$ levels near the current limits of $\mathrm{H}_{\mathrm{I}}$ mapping) to effectively exclude star formation except within anomalously high-density cloud complexes. Previous claims of a star formation threshold near the $D_{25}$ radius appear to have marked a transition in the typical mode of cloud collapse leading to star formation. The magnitude of this transition was overly accentuated by the use of $\mathrm{H} \alpha$ as a SFR tracer.

\subsection{Does Galaxy Type or Environment Matter Most?}

It appears that galaxy type is of little importance with regard to the genesis (and support, if SF is not episodic) of sparse, spatially extended star formation, given the detection of Type 1 XUV-disks associated with spiral systems of all types in the S0-Sdm range ( $\S 3.3 .1)$. Rather, what appears crucial is the means to supply a rich, widely distributed reservoir of gas, whether through (tidal) removal of the interstellar medium from the ordinary disk or infall/accretion processes. Clearly, in many cases (e.g., NGC 1512, M81) galaxy interaction is responsible. For the majority of our XUV-disk class, the source of outer disk gas is not so obvious. We do see evidence for some type of external perturbation (HVCs; low-mass, LSB companions) in $75 \%$ of Type 1 XUV disks and in about half of the Type 2 objects, but it is unclear if this minor level of perturbation can provide the needed amount of gas to the outer disk. It seems more likely that the minor external perturbation could be enough to stimulate XUV-disk SF if the gas were already present, but quiescent.

Gaseous accretion from the IGM is also expected (e.g., Keres et al. 2005) in two dissimilar modes, hot and cold. Cold-mode accretion, in which the gas is not shock heated to the virial temperature of the system, is thought to dominate for lower mass halos $\left(\log M_{\text {baryonic }}<10.3 M_{\odot}\right.$, or $\left.M_{\text {halo }} \lesssim 11.4 M_{\odot}\right)$. The observed lack of Type 2 XUV-disk systems at $L_{K} \gtrsim 10^{9} L_{\odot}$, and consequent skew toward late-type disks, hints that perhaps these galaxies are those that are now growing substantially through efficient cold accretion gas feeding along IGM filaments. Looking from a galaxy-wide integrated perspective, we showed in $\S 3.3 .2$ (Fig. $9 b$ ) that XUV-disks are nearly twice as gas-rich as non-XUV-disk galaxies of similar Hubble type. This could indicate that whatever mechanism is supplying the gas needed for XUV-disk formation has been working for a prolonged duration to achieve this level of richness, as it seems unlikely that a shortterm event would generally be capable of doubling the gas content without leaving signs of a past major merger. The elevated $M_{\mathrm{H}} / L_{B}$ values for XUV-disk galaxies are not caused by a decrease in $L_{B}$ at fixed $T$-type.

Returning to the issue of galaxy interactions and/or potentially minor perturbations, a few comments are appropriate. We mentioned above how NGC 1512 and M81 show outer disks that have been tidally structured. Other systems (e.g., NGC 2782, NGC 4483, NGC 6239, NGC 1140) appear to have suffered a quite significant, but dissimilar galaxy interaction, such that the spatially extended star formation we note as XUV-disk structure comes only from tidal or peculiar features rather than a true disk. Will such objects return to being otherwise normal galaxies after the current burst of XUV-disk SF ceases? Many ordinary galaxies having peculiar $\mathrm{H}$ I arms are found in the literature, objects which could have already experienced a burst of SF in the (tidal) $\mathrm{H}$ I features. Finally, some XUV-disks show evidence for perturbation of a less encompassing variety. Bekki \& Chiba (2006) numerically modeled the influence of dark subhalos in orbit around a galaxy, finding that fine structure and holes can be created in the extended $\mathrm{H}$ I distribution provided that the characteristic subhalo is massive enough. We consider this hypothesis viable for features of a disorganized nature, but note that many of the XUV-disks show large-scale spiral morphology in their outskirts. Dark impact such as that modeled by Bekki \& Chiba (2006) could not produce such coherent gaseous filaments nor the following star formation.

As it may provide a clue to the specific mechanisms which promote or trigger outer disk star formation in the (azimuthally averaged) subcritical regime once gas is in place, we note that some extended $\mathrm{H}$ I disks are known that do not exhibit any sign of recent star formation, such as NGC 2915 and NGC 925 (as noted in Boissier et al. 2007). Given that galaxies such as these exist, will they remain stable? Have they formed extreme outer disk stars before, then transitioned to a quiescent phase? Or did they recently acquire their gas, on a timescale too short for SF to have become organized? We do not yet have a good handle on which of these scenarios is correct, or even if a single explanation suffices. Even if we assume that XUV-disk SF is episodic, we cannot yet constrain the duty cycle except to say that some bursts seem to last for a minimum of a few $\times 10^{8} \mathrm{yr}$ (considering the detection of both $\mathrm{H} \alpha$ and aging UV sources), and that the relative amount of time spent in the quiescent phase is probably small for galaxies with extended $\mathrm{H}_{\mathrm{I}}$. This last assertion is based on the fact that only a handful of the extended $\mathrm{H}$ I galaxies of which we are aware in the present sample (Table 1) do not show XUV-disk structure. Note that there could also be galaxies in which XUV-disk SF has largely consumed the available outer disk gas, leaving only an imprint in the radial distribution of stellar mass and characteristic age (e.g., Muńoz-Mateos et al. 2007). If inside-out disk formation models are accurate, most spiral galaxies may have already experienced XUV-disk SF to at least some degree.

\subsection{XUV-Disks and LSB Galaxies}

The preceding discussion focused on the possible relation of XUV SF and ordinary galaxies, but there are also reasons to consider atypical objects. The apparent similarity of physical conditions (low $\Sigma_{\mathrm{SFR}}$, low metallicity, sparse morphology) in Type 1 XUV-disk environments and massive LSB (MLSB) galaxies makes comparison of these objects compelling. Do MLSB galaxies represent an extreme variant of the XUV-disk class?

Barth (2007) recently challenged the current understanding of the prototype MLSB Malin 1's galactic structure based on Hubble Space Telescope (HST) WFPC2 I-band (F814W) imaging, questioning the central surface brightness and scale length of the disk. He concluded that a brighter and more steeply declining disk component had been missed in ground-based images, with the bulge actually more compact than previously thought. Comparing his revised estimates for Malin 1's disk parameters with those of other S0 galaxies (Aguerri et al. 2005), Barth reported that the underlying inner disk was unremarkable, and not faint enough to qualify Malin 1 as an LSB galaxy. GALEX observations of Malin 1 show faint UV emission distributed over the extent of 
the $\mathrm{H}$ I disk, such that Malin 1 certainly should be classified as an XUV-disk according to our criteria. NGC 262 is a MLSB galaxy in the GALEX Atlas (Gil de Paz et al. 2007a) which is similar to Malin 1, but not included in our sample because of distance. MLSB objects could merely be the high-mass tail of the XUVdisk population. No matter what their classification, MLSB galaxies again demonstrate the pervasive nature of SF, even in environments that are characterized by overall low density.

There may also be lessons to be learned from XUV-disks in the context of LSB galaxies at the low end of the mass spectrum, namely LSB dwarf galaxies. Heller et al. (1999) completed an $\mathrm{H} \alpha$ survey of LSB dwarfs in the Virgo Cluster, finding small integrated SFRs (several $\times 10^{-3}$ to $10^{-2} M_{\odot} \mathrm{yr}^{-1}$ ), with the bulk of their emission coming from a few discrete $\mathrm{H}$ II regions per target (if at all). Just as the low $\Sigma_{\text {SFR }}$ values characterizing the XUV-disk zone in giant spirals can lead to a typical probability $<1$ of catching a stellar cluster still young enough to produce an $\mathrm{H}$ II region, we caution that some fraction of the LSB dwarf population may have recent SF that has been missed entirely, or a time-averaged SFR that has been underestimated, due to stochastic effects. A comprehensive survey of LSB dwarf galaxies in the UV is needed to assess this possibility.

\subsection{XUV-SF and the Process of Disk Building}

What is the evolutionary impact of XUV-disk star formation over cosmological time periods, and how is this influence reflected in the non-UV properties of a galaxy? Clearly, the answer will vary depending on the length of time over which the XUV-disk star formation has been sustained, and the degree of SF intermittency during this time. The high specific star formation rate (sSFR) of Type 1 XUV-disks beyond the threshold contour and overall for Type $2 \mathrm{XUV}$-disks suggest that XUV-SF is an important disk-building mechanism. This process is much harder to study in the distant universe (e.g., Trujillo \& Pohlen 2005) at an epoch during which the bulk of galaxy disks were assembled.

There has been much contemporary progress on evolved stellar populations in the outer disk, including insights possibly linked to XUV-disk star formation. The low surface brightness radial extent of the evolved stellar disk is now known to be much larger than previously thought, and exhibits a variety of structure in its surface brightness profile shape.

Ibata et al. (2005) reported an exponential disk-like component in M31 extending to at least $40 \mathrm{kpc}$ radius (about twice the previously known size) having kinematics consistent with the expected circular motion at the radii probed. The stellar population of a small section within the outer M31 disk was found to have an intermediate age of 4-8 Gyr by Brown et al. (2006). A vast, purely exponential disk of very low surface brightness was also recently suggested in NGC 300 by Bland-Hawthorn et al. (2005), reaching out to 10 times the optical scale length.

Simple exponential profiles, however, are rare (10\% according to Pohlen \& Trujillo 2006). Disk galaxies with profiles well described by a broken exponential are now thought to constitute a majority of the disk population (Pohlen et al. 2002, 2004; Pohlen \& Trujillo 2006; also Hunter \& Elmegreen 2006 for Im galaxies) — perhaps reflecting a change of star formation and disk assembly processes at the break radius, and generally emphasizing the complicated nature of disk-building processes. Of the broken exponentials, some profiles are up-turned at the break radius, and others are down-turned. Down-turned broken exponentials are easier to explain in terms of a SF threshold framework (or, alternatively, the new view of reduced SF efficiency due to large scale zones with $Q>1$ and reliance on secondary, nongravitational cloud collapse mechanisms therein). They also completely replace the sharp radial cutoffs proposed by van der Kruit (1979). Of perhaps even more relevance to XUV-disks, Erwin et al. (2005) and Pohlen \& Trujillo (2006) discovered "antitruncated" spiral galaxies, disk systems with surface brightness profiles best fit by a double exponential in which the outer disk has a larger scale length than the inner disk.

Naively, one would suppose that up-turned, antitruncated optical disks might be associated with galaxies that are extended in terms of their gas distribution, whereas down-turned galaxies have a consistently declining $\Sigma_{\text {gas }}$ profile. This originally suggested to us (Thilker et al. 2005b) that antitruncated disks might be the evolved counterparts of XUV-disks. Tantalizingly, the Elmegreen \& Hunter (2006) SF model predicts double exponential SFR profiles with a down-turn in the outer disk for the case of a purely exponential radial decline in average $\Sigma_{\text {gas }}$, but also predicts purely exponential or even broken, up-turned profiles in the case of galaxies having a more gradual $(1 / R)$ drop in the gas distribution. They further confirmed the expectation that the stellar mass surface density profiles built up by SF over many Gyr have a form resembling the predicted SFR profile.

However, the observed relation between XUV-disk SF and optical profiles remains unclear at this point. In XUV-disk galaxy NGC 300, the GALEX surface brightness profiles in FUV and NUV exhibit a down-bending break near the $D_{25}$ radius (Fig. 3 of Gil de Paz et al. 2007a), but Bland-Hawthorn et al. (2005) report a pure exponential form of the underlying evolved stellar disk out to well beyond this radius. Some of our sample galaxies were studied optically by Pohlen \& Trujillo (2006). UGC 04393, optically fit with a pure exponential by Pohlen \& Trujillo, is classified as a mixed-type XUV-disk. In the case of Type 1 XUV-disk NGC 3359, categorized as down-bending optically (their type II-AB) by Pohlen \& Trujillo, we observe well-defined outer spiral structure in the GALEX UV imaging. Even more perplexing is NGC 2541, which we designate as a Type 2 XUV-disk and Pohlen \& Trujillo call a down-bending optical exponential (their type II-CT). Five of the up-bending profile galaxies of Pohlen \& Trujillo (their type III and III-d) are included in our survey sample. They are NGC 1084, NGC 2976, NGC 4030, NGC 5713, and NGC 5806. Only one of these targets (NGC 2976) exhibits clear disklike properties at large radii in the optical (III-d), but nevertheless we find it intriguing that none of these galaxies are classified as XUV-disks. This could imply that, if Pohlen \& Trujillo type III galaxies are evolved XUV-disks, there comes a time when XUV-disks run out of gas to convert into stars, or at least that quiescent periods last for a few hundred Myr.

In any case, complex outer disk optical profiles demonstrate that SF in low-density outskirts of a galaxy is commonplace, or more precisely that it was at some time in the past, and that such outer disk SF is supported to varying degrees (perhaps determined by the availability of gas through accretion and/or interaction).

\subsection{Morphological Structuring of XUV Emission and $\mathrm{H}$ I}

Typically, the spatial extent of outer disk SF matches the apparent $\mathrm{H}$ i size of a galaxy evaluated at the sensitivity attainable with the VLA at GALEX resolution $\left(N_{\mathrm{H}_{\mathrm{I}}} \sim 10^{20} \mathrm{~cm}^{-2}\right)$, with UV peaks generally following large-scale $\mathrm{H}$ i emission structures, but not necessarily corresponding to $\mathrm{H}$ I clumps individually. This is expected, however, as the mechanical luminosity of the clusters (from SNe and stellar winds) should be sufficient to create holes or bubbles in the local distribution of gas and effectively break down the Schmidt law on small scales. Again, 
although an extended $\mathrm{H}$ i distribution appears to be a XUV-disk prerequisite in all but the most bizarre cases (e.g., NGC 4254), we reiterate that there are extended $\mathrm{H}$ I galaxies without evidence of recent SF in their outer disk (NGC 925, NGC 2915).

Despite the large area over which XUV-disk star formation proceeds, established by the H I disk size and CNM content, the total outer disk SFR in most XUV-disk galaxies is modest. The low covering factor of the UV-emitting sources explains the modest total XUV-disk SFRs, even if on small scales the star formation surface density in clump complexes becomes significant. Even when cast into a fractional form ( $\left.\mathrm{SFR}_{\mathrm{XUV} \text {-disk }} / \mathrm{SFR}_{\text {galaxy }}\right)$, the XUV-disk star formation is generally limited to a few percent of the galaxy-wide integrated level, with the exception of Type 2 XUV-disks. One could argue that the small total and fractional SFRs associated with XUV-disk environments means that the SF occurring there is unimportant in a cosmological sense. It is true that at the present epoch XUV-disk activity is a secondary mode of baryonic transformation from gas to stars, when averaged over the galaxy population as a whole. Nevertheless, it has profound consequences for the future chemical and photometric evolution of the outer disk, and provides a snapshot of how disk galaxy formation might occur in general, via building of the stellar disk from the inside-out.

We observe a wide variety of UV morphology among the XUV-disks described in Appendix A and displayed in Fig. Set 16. The overall morphological distribution is represented by our two types of XUV-disk structure (Type 1 and Type 2), but there is subtlety, with some features observed in both Type 1 and Type 2 galaxies. Here we highlight some of the morphological diversity. The GALEX data show wispy extensions of the inner disk at markedly lower intensity (NGC 300, NGC 4414), spiral-arm continuation with gradual fading (NGC 628, NGC 3344, M 101), warped $\mathrm{H}$ I disks with prominent internal structure in either ringlike or spiral form (NGC 2841, NGC 5055, M83), tidal filaments (NGC 772, NGC 1512, NGC 2782, M81, NGC 6239) or interaction debris (NGC 4438), "filled" envelopes with/without spiral substructure (NGC 1672, NGC 1042, NGC 3621, NGC 4625, NGC 6902-with fading, too), very LSB with isolated clumps (NGC 2146A, NGC 3185), unambiguous well-isolated spiral arms (NGC 3359), swept/lopsided appearance (NGC 4254), and outer spiral structure unrelated to optical appearance but also seen at inner radii (NGC 4383).

Finally, it is interesting to compare the clumpy XUV-disk emission against compact features dominating inner disk UV morphology. Preliminary measurements indicate that the distribution of peak surface brightness and luminosity for outer disk clumps is substantially fainter than for compact structures occupying the inner disk. Indeed, many of the XUV clumps have peak surface brightness comparable to the interclump emission of the main disk. Perhaps the clumped outer disk emission is more similar to inner disk diffuse UV light - now thought to be composed of dissolved clusters (Pellerin et al. 2007). The process of "infant mortality" is understood to occur on rather short timescales, conceivably even less than 10-25 Myr. In principle, the disruption of clusters may begin with the first $\mathrm{SNe}$ in a star-forming complex. Our Monte Carlo simulations suggest that the majority of sources in the outer disk should be older than this very young age. The combination of moderate age (with clumps being dominated by $\mathrm{B}$ stars rather than $\mathrm{O}$ ) and a relatively diffuse ISM, which might encourage cluster disruption, leads us to believe that infant mortality of clusters through disruption of the natal star-forming cloud may be quite effective in the XUV-disk environment. We have already obtained HST UV-optical imagery of locations in M83 and NGC 5055 which will be used to test this hypothesis.

\section{CONCLUSIONS AND SUMMARY}

We searched for extended ultraviolet disk (XUV-disk) galaxies in the local universe, following the discovery of extreme outer disk star formation in M83 and NGC 4625. Our initial survey of 189 spiral galaxies within 40 Mpc observed by GALEX with uniform (surface brightness) sensitivity showed evidence for two varieties of XUV-disk structure. Type 1 XUV-disks similar to our prototypes (e.g., M83, NGC 4625), have UV-bright cluster complexes (often arranged in spiral-like features) at extreme galactocentric distances, where the mechanisms leading to initial cloud collapse (and eventual gravitational instability) begin to phase out. Type $2 \mathrm{XUV}$-disks are dominated by a large, outer, blue LSB zone having rather high specific star formation rate. In Type 2 disks the SF does not necessarily reach to extreme disk radii, and can even show a sharp outer edge. The actual galaxy population exhibits a continuum of properties, even in the case of XUV-disk star formation. The main two types may be viewed as illustrative of the modes of galaxy evolution via insideout disk formation (Type 1 gradual, Type 2 extreme/nonlinear), but are not mutually exclusive.

The apparent, present-day incidence of XUV-disk star formation corresponds to $\gtrsim 20 \%$ of spiral galaxies having Type 1 (M83-like) structures and $\sim 10 \%$ Type 2 XUV-disk morphology. Actual incidence over cosmological periods could be even higher if XUV SF is episodic, or if it has already happened in some fraction of the galaxy population not classified as XUV-disks. We find that Type 1 XUV-disks span the entire range of Hubble types considered in our survey, while Type 2 XUV disks appear to be preferentially late-type/low-mass spirals. Global characteristics are similar to that of the general galaxy population, albeit with mildly increased gas richness and hints that Type 1 XUVdisks may be stimulated by minor perturbation. The lack of other notable changes in integrated properties between the XUV-disk galaxies and the entire sample suggests that perhaps XUV-disk building is a part of the natural process of galaxy growth which is inherently tied to the environmental influences acting on on any particular object. Recent/ongoing interaction events and a high specific rate of gas accretion are two possible triggers for XUVdisk formation.

GALEX (Galaxy Evolution Explorer) is a NASA Small Explorer, launched in 2003 April. We gratefully acknowledge NASA's support for construction, operation, and science analysis for the GALEX mission, developed in cooperation with the Centre National d'Etudes Spatiales of France and the Korean Ministry of Science and Technology. A. G. d. P. is financed by the MAGPOP EU Marie Curie Research Training Network and partially by the Spanish Programma Nacional de Astronomía y Astrofísica under grants AYA2003-01676 and AYA2006-02358.

This research has made use of the NASA/IPAC Extragalactic Database (NED). We acknowledge the use of the HyperLeda database (http://leda.univ-lyon1.fr). The Digitized Sky Surveys were produced at the Space Telescope Science Institute under US Government grant NAG W-2166. Funding for the SDSS and SDSS-II has been provided by the Alfred P. Sloan Foundation, the Participating Institutions, the National Science Foundation, the US Department of Energy, the National Aeronautics and Space Administration, the Japanese Monbukagakusho, the Max Planck Society, and the Higher Education Funding Council for England. Some images presented in this paper were obtained from the Multimission Archive at the Space Telescope Science Institute 
(MAST). STScI is operated by the Association of Universities for Research in Astronomy, Inc., under NASA contract NAS526555. Support for MAST for non-HST data is provided by the NASA Office of Space Science via grant NAG5-7584 and by other grants and contracts. This research has made use of the
NASA/IPAC Infrared Science Archive. This publication makes use of data products from the Two Micron All Sky Survey.

Facilities: GALEX, CTIO:2MASS, FLWO:2MASS, Sloan, PO:1.2m, UKST

\section{APPENDIX A}

\section{NOTES ON INDIVIDUAL XUV-DISK GALAXIES}

We now comment on each XUV-disk galaxy in our GALEX survey, and provide background information from the literature when relevant. Figure Set 16 shows GALEX, visible (DSS2-red and SDSS DR5 gri color composite when available), and 2MASS- $K_{S}$ imaging data for each galaxy described in this section. We have grouped the galaxies according to their XUV-type, first the Type 1 disks, then mixed-type objects, and finally Type 2 XUV-disks. In the print edition, we only show two galaxies per group as an example; see the electronic version of the Supplement for the complete Figure Set 16.
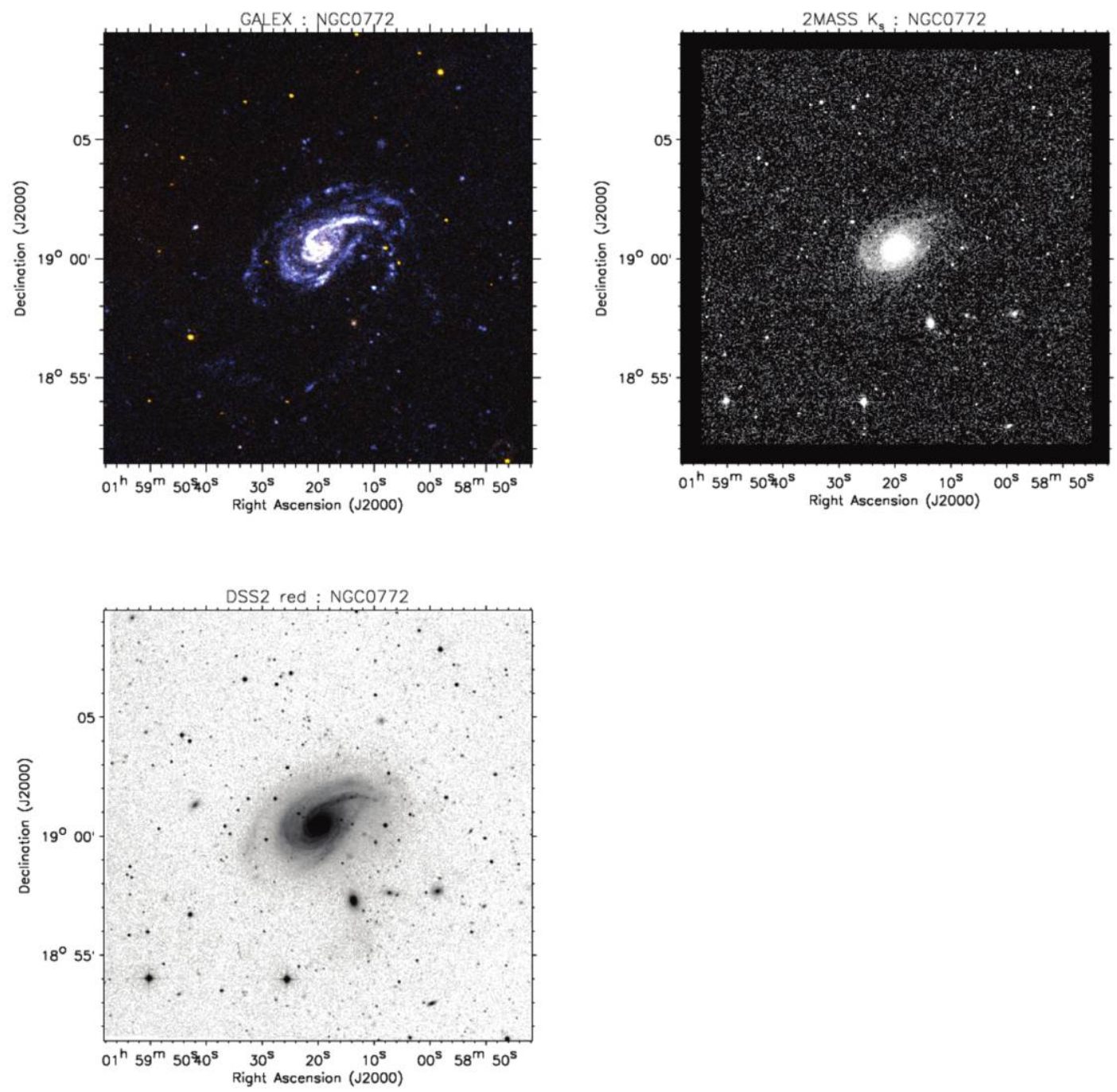

FIG. 16.3

FIG. SEт 16.-Multiwavelength imaging of XUV-disk galaxies. For each object we present $(a)$ GALEX FUV and NUV, $(b)$ 2MASS- $K_{s},(c)$ DSS2-red, and $(d)$ SDSS-DR5 imaging (when available). In the case of SDSS, we display the gri bands as blue, green, and red channels. The extent of all images is 3 times the $D_{25}$ size of the galaxy. The print figure only presents two galaxies of each XUV-disk variety, as a guide to content. NGC 772 and NGC 4254 (M99) are shown as examples of Type 1, UGC 10445 and NGC 7418A for mixed-type objects, and NGC 2090 and NGC 2541 for Type 2 XUV-disks. [See the electronic edition of the Supplement for complete set of Figs. 16.1-16.54.] 

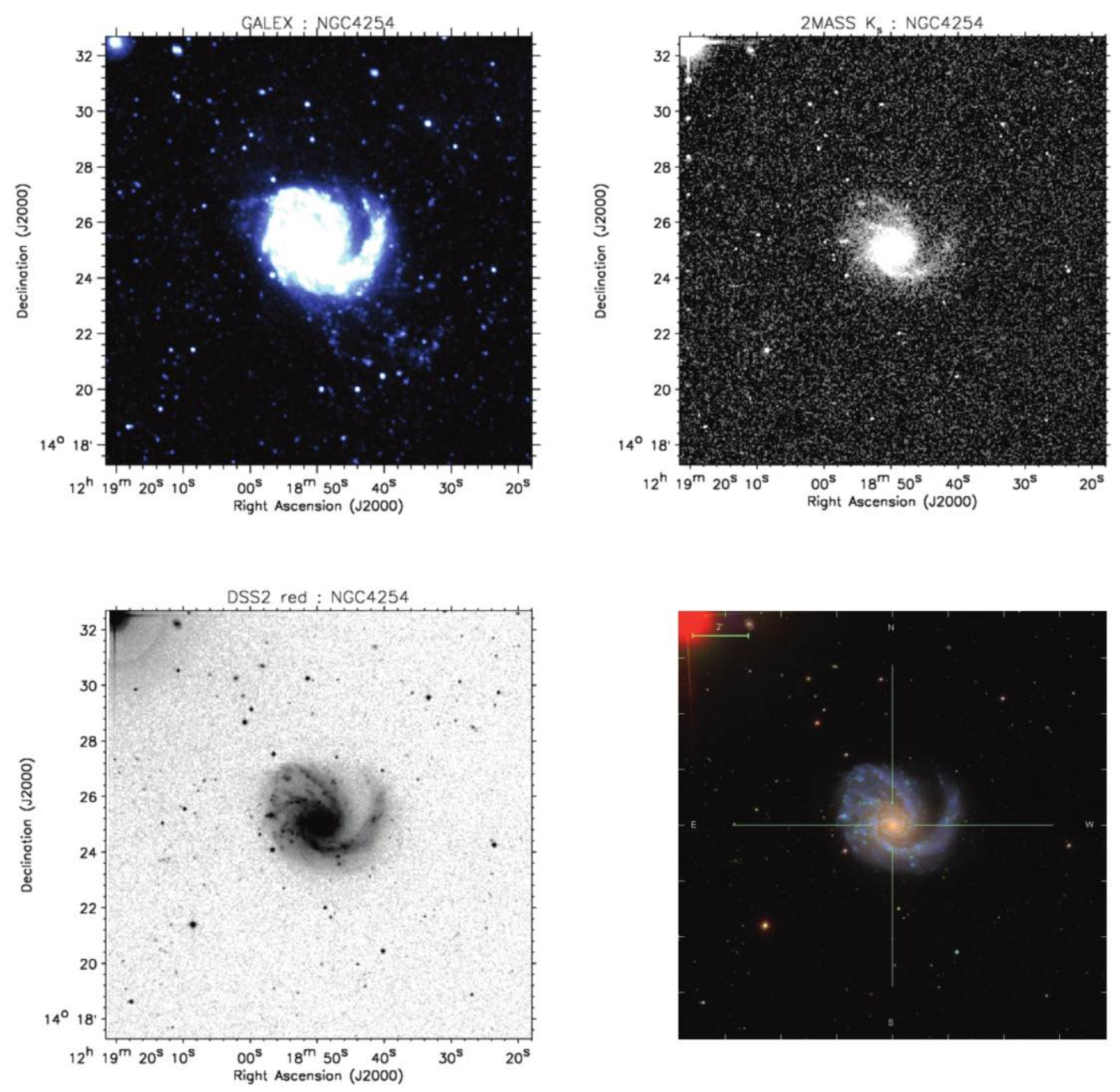

FIG. 16.18

\section{A1. TYPE 1 XUV DISKS}

$N G C 300$ (Fig. 16.1)._-GALEX imaging of this SA(s)d galaxy reveals a bright inner disk filled with SF complexes, in addition to an outer disk occupied by low surface brightness, wispy arcs of clumpy XUV emission. Radial surface brightness profiles in FUV and NUV from the GALEX Atlas (Gil de Paz et al. 2007a) indicate a downward break in the slope of the profile at the $D_{25}$ radius. NGC 300 is known to have a large $\mathrm{H}$ I envelope $\left(D_{\mathrm{H}_{\mathrm{I}}} \sim 61^{\prime}\right.$; Puche et al. 1990) with a warp beginning at radii of $10^{\prime}$. The outer parts of this $\mathrm{H}_{\mathrm{I}}$ distribution are only very sparsely populated with UV clumps. Some of the XUV disk clusters associated with NGC 300 can be recognized on the DSS2-red plate, using the UV imagery as a prior. Bland-Hawthorn et al. (2005) recently reported a disklike component of the resolved stellar population out to 10 radial scale lengths $\left(24^{\prime}\right.$, or $\left.2.2 R_{25}\right)$ in this galaxy. A causal relation between the outer disk star formation evident from our GALEX data and the extended disk of Bland-Hawthorn remains speculative, however. A member of the Sculptor group, NGC 300 is frequently described as a twin of M33. It dominates a "loose quartet" (Karachentsev et al. 2003) of Sculptor galaxies including NGC 55, ESO 410-05, and ESO 294-10 (later two are not listed in the NOG catalog; Giuricin et al. 2000).

NGC 628 (Fig. 16.2).- This SA(s)c galaxy has received attention (along with NGC 6946 and NGC 1058) as an example of a spiral with faint H II regions populating the outer disk (Ferguson et al. 1998a, 1998b; Leliévre \& Roy 2000). GALEX imaging shows UV clumps at the positions of each previously known outer disk $\mathrm{H}$ II region, as well as numerous additional UV-only complexes located at modestly larger radii (at least to $10.3^{\prime}$, maybe farther to the east and northwest). The census of recent star-forming sites (even in areas populated by outer disk $\mathrm{H}$ II regions) is therefore more complete in our GALEX data. Spiral structure is apparent in the XUV disk, although the covering factor of SF detected complexes is small. The XUV-disk spiral structure appears to be a continuation of the inner disk pattern, although perhaps more tightly wound. The H I distribution of NGC 628 is known to be extended (Kamphuis \& Briggs 1992; Auld et al. 2006) within a diameter of approximately $40^{\prime}$ at a limit of $N(\mathrm{H} \mathrm{I})=2.2 \times 10^{18} \mathrm{~cm}^{-2}$. Kamphuis \& Briggs (1992) noted the presence of anomalous high-velocity gas. Smaller companions are UGC 01104, UGC 01171, UGC 01176 (DDO 13), UGCA 20, and KDG 10, as well as DW 0137+1541 (Briggs 1986). NGC 628 is paired with the disturbed, polar-ring galaxy NGC 660, which also has companions. 

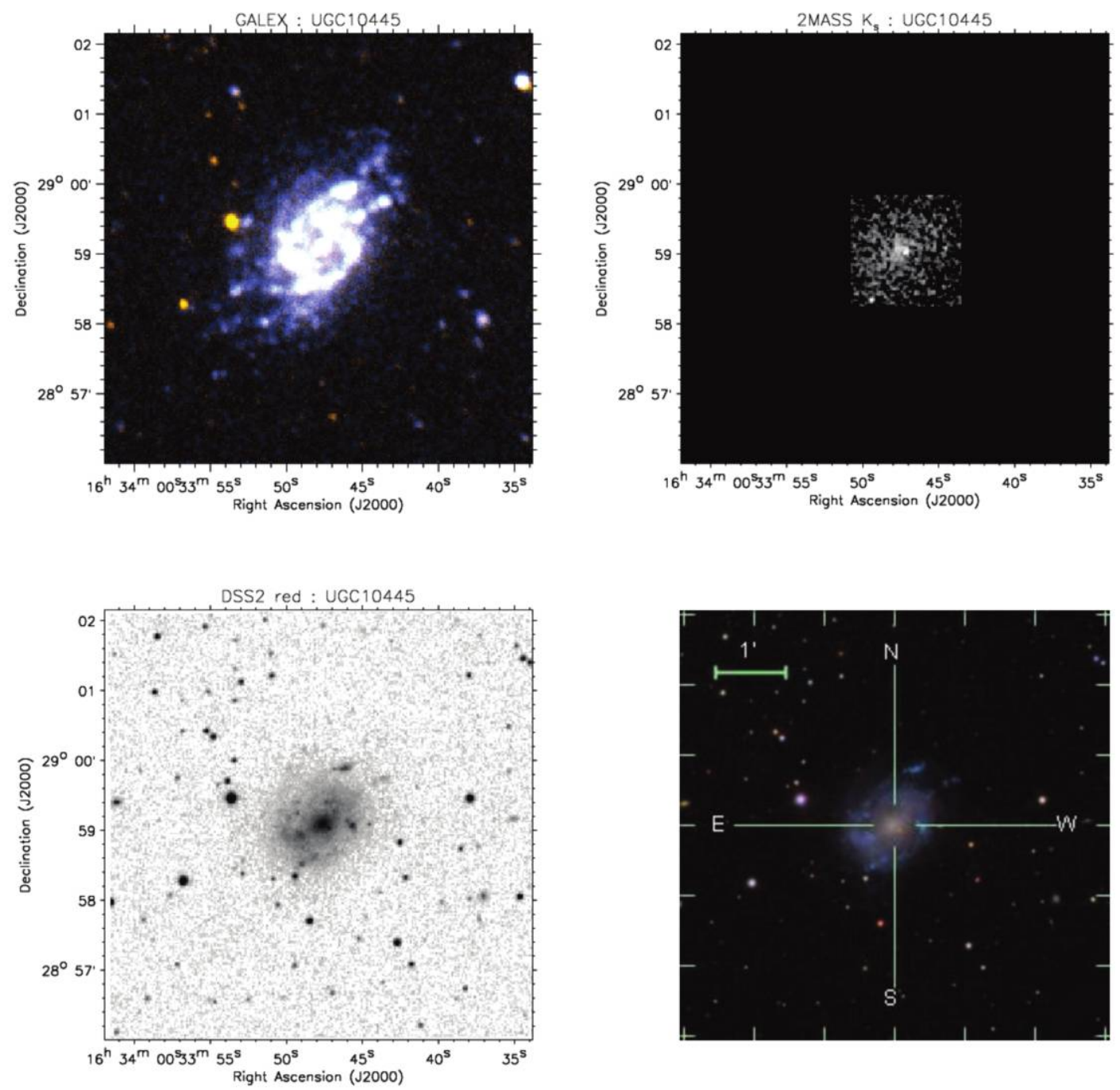

FIG. 16.36

$N G C 772$ (Fig. 16.3).-GALEX imaging of this SA(s)b galaxy shows a complex, asymmetric spiral structure with an anomalously bright arm extending to the northwest of the nucleus for approximately $3^{\prime}$. This UV morphology (and the visible appearance) is highly suggestive of past or present galaxy interaction, probably with NGC 770 ( $3.3^{\prime}$ southwest). Such excitation of one-armed spiral modes has been discussed by Taga \& Iye (1998) and Lovelace et al. (1999). The inner part of the NGC 772 disk is surrounded by tightly wrapped, generally continuous spiral features, which attain their greatest extent $\left(7.1^{\prime}\right)$ to the south of the galaxy. NGC 772 dominates a NOG group (NOGG 124) of three bright galaxies. Zaritsky et al. (1997) further associate three lower mass companions with NGC 772. Of these, NGC 772b appears optically disturbed (Gutierrez et al. 2006). NGC 772 has a comparatively large H I disk (Rao \& Briggs 1993; Iyer et al. 2001).

NGC 1042 (Fig. 16.4).- - This SAB(rs)cd NLAGN galaxy has along its perimeter (predominately to the east and west) an annular zone of reduced SF intensity, for which both GALEX and H $\alpha$ data (SINGG; Meurer et al. 2006) indicate a population of distinctly lower luminosity SF regions. SDSS data does not show the vast majority of emission features detected by GALEX in this zone. NGC 1042 belongs to a group of 11 galaxies dominated by NGC 988 (Giuricin et al. 2000), but also containing NGC 991, which we have classified as a Type 2 XUV disk. The H I disk of NGC 1042 has been studied in detail by Kornreich et al. (2000), who find that the gas distribution is asymmetric, and conclude that this resulted from interaction with NGC 1052 (also suggested by van Gorkom et al. 1986). Although it reaches past the optical disk emission, the relative $\mathrm{H} \mathrm{I}$ extent is not particularly large $\left(D_{\mathrm{H}_{\mathrm{I}}} / D_{25}=1.7\right.$; Kornreich et al. 2000) in comparison to other spirals. The outer gas disk also does not appear substantially structured into filaments at $20^{\prime \prime}(\sim 1 \mathrm{kpc})$ resolution. The net effect of these two factors is that the XUV-disk emission in NGC 1042 does not attain truly extreme galactocentric distances, such as found in many of our other objects.

NGC 1512 (Fig. 16.5).- This SB(r)ab galaxy is in the midst of a close interaction with NGC 1510 (located $5^{\prime}$ to the southwest, projected inside of the XUV-disk). The UV morphology of the pair is dominated at high surface brightness by an inner SF ring in NGC 1512 and starburst activity in the nuclear region of each galaxy. A single spiral arm begins at the northeast edge of the SF ring and wraps continuously around NGC 1512 for nearly $540^{\circ}$ in azimuth, eventually reaching a galactocentric distance of $11.4^{\prime}$ southwest of the galaxy. H I synthesis imaging (M. Thornley 2007, private communication) shows that the spatial distribution of atomic gas and 

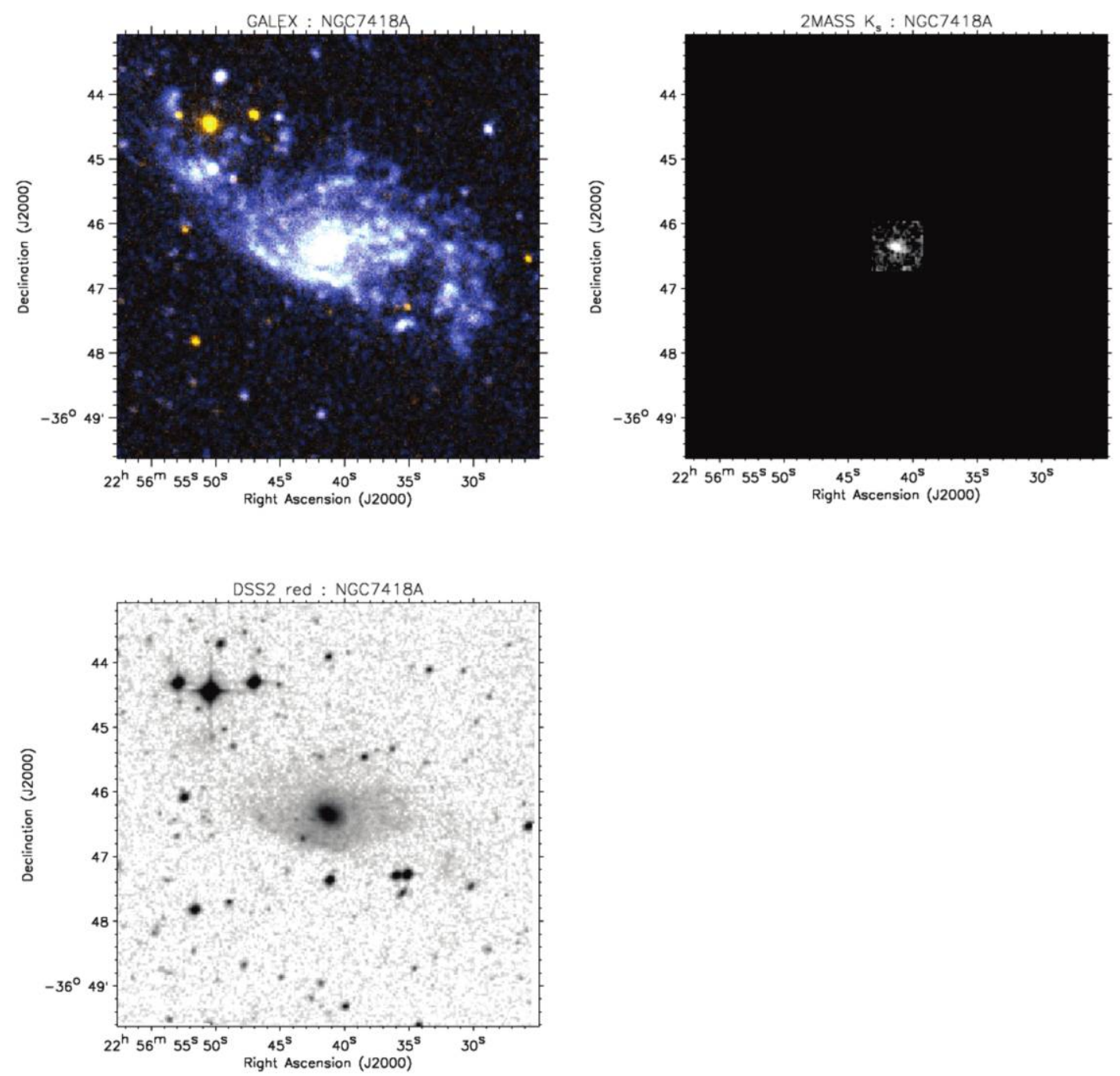

FIG. 16.37

UV-bright complexes are tightly correlated. The tip of the continuous, winding XUV arm coincides with the position where the H I arm becomes undetected. Using the $\mathrm{H}_{\mathrm{I}}$ image as a prior, we can discern what may be a continuation of this arm directly to the west of NGC 1512, where we find a UV clump (at 12.6') coincident with a local maximum in an elongated $\mathrm{H}$ I feature oriented north-south. Arcs of UV-bright complexes can be found associated with NGC 1510, just to the north of this SA0 pec; H II BCDG companion. Disorganized UV filaments can be found scattered northwest of NGC 1512, which cannot be confidently associated with either galaxy in the pair. Perhaps these features represent SF occurring in tidally disturbed material. The large extent of the outer spiral features in NGC 1512 was noted by both Hawarden et al. (1979) and Sandage \& Brucato (1979) using optical observations, although the degree of recent star formation activity in these locales is a new GALEX result. The NGC 1512 group also contains NGC 1487.

NGC 1672 (Fig. 16.6).- This southern (R'1:)SB(r)bc Seyfert 2 galaxy has an XUV-disk with a high surface covering factor, composed of spiral segments continuing outward from the prominent inner disk arms. The elongation and position angle of the outer disk is similar to that of the galaxy's bar, and differs from the optical isophotal contours at intermediate surface brightness. This may be the signature of SF occurring in a warped H I disk. Only NUV observations are available for this target, making the task of identifying the most spatially extended complexes more difficult than usual. Sandage \& Bedke (1994) previously noted faint outer disk structure in this galaxy on the basis of deep optical prints. NGC 1672 is the main galaxy in a small group containing at least eight galaxies (from NOG catalog). The NGC 1672 group is part of the yet larger Fornax Wall and Dorado group.

NGC 2146A (Fig. 16.7).- This SAB(s)c: galaxy has a very low surface brightness outer disk, which is most apparent to the west and northeast. NGC 2146A is isolated, according to the NOG catalog of galaxy groups.

NGC 2710 (Fig. 16.8).- This SB(rs)b galaxy has an inner disk accentuated by bright UV emission from a bar and adjoining spiral arms. The spiral arms fade in surface brightness and become indistinguishable from a fainter, clumpy distribution filling the optical extent of the galaxy. An arclike, XUV spiral arm is found beyond the northwest limit of the inner disk about $1.5^{\prime}$ from the galaxy nucleus. SDSS observations present a similar morphology as GALEX, but do not show the northwest feature. NGC 2710 is isolated according to the NOG clustering analysis. 

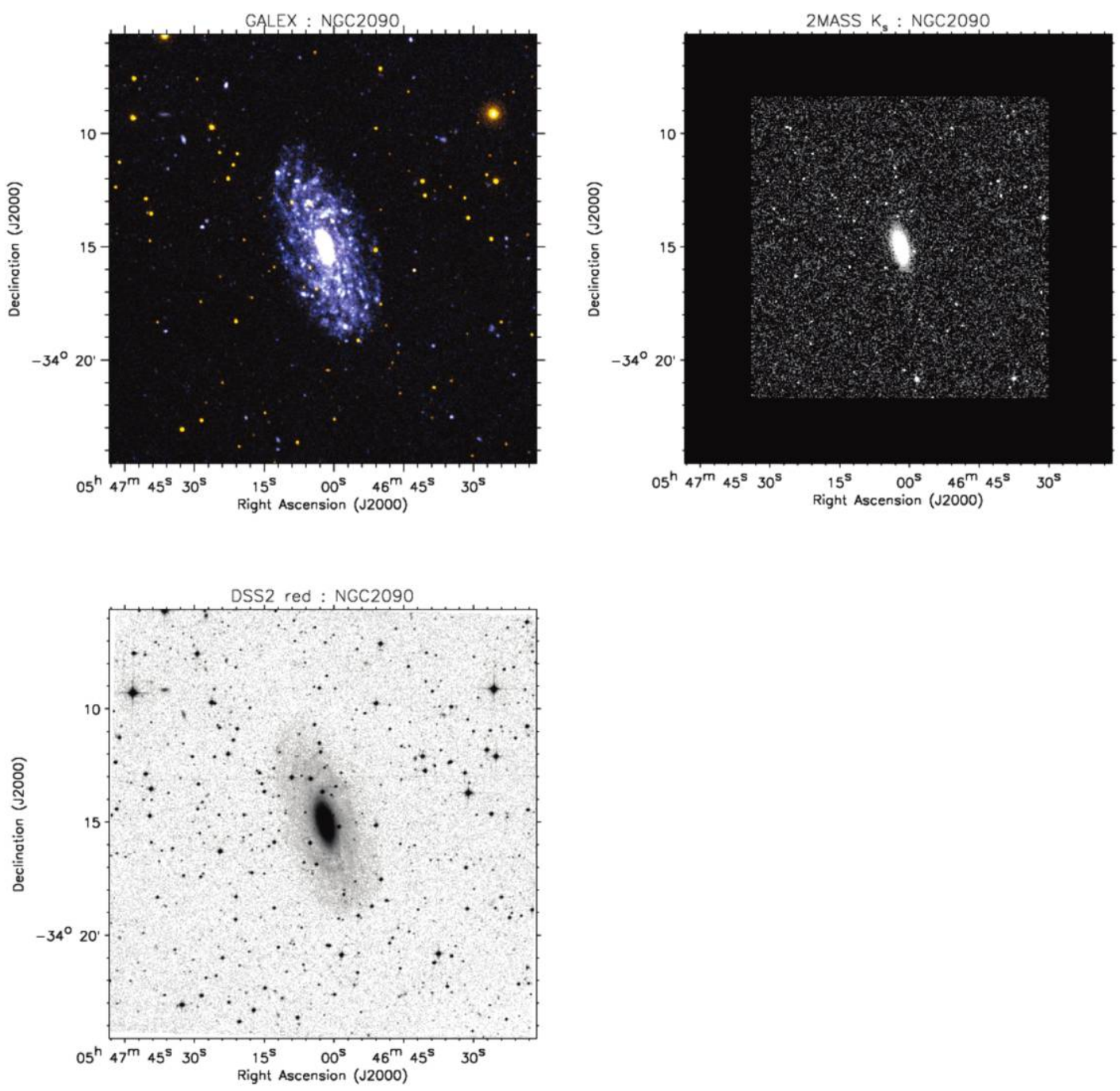

FIG. 16.40

NGC 2782 (Fig. 16.9). - The clearly disturbed ultraviolet and optical morphology of this SAB(rs)a; Seyfert 1 starburst galaxy has been attributed to a previous galaxy merger by Smith (1991). The merger remnant exhibits a very large, double-sided H I tail (Smith 1991, 1997; Noordermeer et al. 2005) which has modest extent directly east of the galaxy (coincident with low surface brightness plume in DSS imaging) but more significantly reaches $5^{\prime}$ to the northwest in the form of a long curving feature. Our GALEX images show clumpy UV emission within the NW tail, matching the morphology of the atomic gas, and a component of more diffuse and redder UV light originating from the eastern $\mathrm{H}_{\mathrm{I}}$ cloud. Smith (1991) reports that LSB $\left(\mu_{B}=25\right.$ mag $\left.\operatorname{arcsec}^{-2}\right)$ optical emission can be seen within the NW tail, but the UV clumps seen in our data are more conspicuous and demonstrate that SF is occurring in the structure. SDSS image data is not sensitive to the young clusters in the NW tail. NGC 2782 is the brightest galaxy in a small group of four objects.

NGC 2841 (Fig. 16.10). - GALEX imaging of this SA(r)b:; LINER Seyfert 1 galaxy shows nearly continuous spiral arms wrapping the outside of a bright, flocculent inner disk. The H I disk is also quite extended (Bosma 1981 and F. Walter et al., in preparation [THINGS]). XUV complexes can be identified out to at least 4 times the $D_{25}$ radius at both ends of the galaxy major axis. These particular features are corroborated by the $\mathrm{H}$ I morphology in the same area (F. Walter et al., in preparation). Star formation activity is ongoing throughout the entire $\mathrm{H}$ I distribution, which exhibits a notable ringlike appearance just outside the optical disk. NGC 2841 is dramatic in terms of the difference in mean UV surface brightness between the inner (optically prominent) disk and the XUV-disk. Some of the XUV-disk complexes in NGC 2841 are luminous enough to be detected individually in SDSS observations. A forthcoming paper by Bianchi et al. (in preparation; see also Bianchi et al. 2007) will discuss population synthesis SED-fitting of these sources. NGC 2841 is a relatively isolated galaxy, as it is only paired with one other object (UGC 04879).

NGC 3031 (Fig. 16.11). - The primary member among one of the most well-studied interacting galaxy groups, this SA(s)ab; LINER Seyfert 1.8 object has extensive tidal arms apparent in the $\mathrm{H}$ i distribution (Yun et al. 1994, 2000). GALEX observations show that the tidal arms host SF at a level greater than appreciated via the $\mathrm{H} \alpha$ tracer (comparing to the emission line image of Greenawalt 1998). SDSS imaging reveals some of the XUV-disk cluster complexes in NGC 3031. Within the large-scale tidal features, molecular gas has been found (Brouillet et al. 1992; Braine et al. 2001). Tidal dwarf galaxies (van Driel et al. 1998; Boyce et al. 2001; Makarova et al. 2002), isolated H II regions (Flynn et al. 1998), and a diffuse stellar population (Sun et al. 2005) were previously known to exist within the 

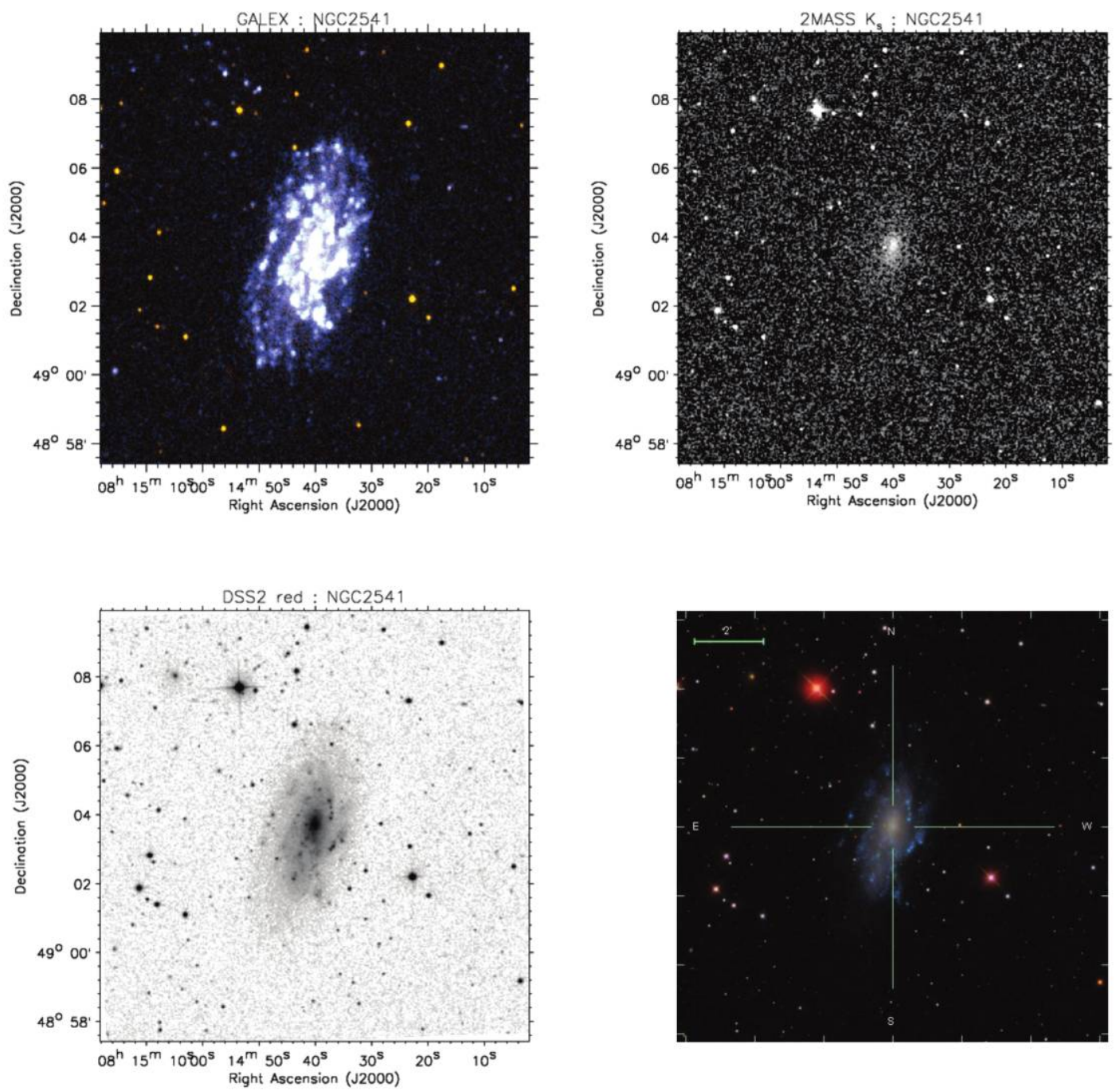

FIG. 16.42

H i streamers. MUV and FUV imagery of NGC 3031 from UIT (Hill et al. 1992; Marcum et al. 2001) illustrated a red bulge and blue $\mathrm{SF}$ arms, but not the XUV-disk complexes.

NGC 3185 (Fig. 16.12).- The ultraviolet morphology of this (R)SB(r)a Seyfert 2 galaxy is dominated by a UV-bright, inner ring and a luminous nuclear source. Outside of this and lying toward the southeast major axis, our GALEX data reveal a string of blue (FUV - NUV) clumps qualifying the galaxy as an XUV-disk, although quite limited in nature. Background galaxies may be confused with some of this emission. NGC 3185 is a member of the group dominated by NGC 3190. SDSS imaging also shows the SF disk in the main disk, but does not detect the XUV clumps.

NGC 3198 (Fig. 16.13).- This inclined SB(rs)c galaxy was studied in some detail following the discovery of an apparent sharp outer edge to the $\mathrm{H}$ I distribution (Maloney 1993; van Gorkom 1991). H I extends to at least 634" radius, about 2.5 times $D_{25}$ radius (Sicking 1997). The H I distribution is asymmetric, with a filament in the northeast portion of the galaxy (Bosma 1981; Sicking 1997; THINGS). GALEX shows that $\mathrm{SF}$ is occurring in spiral waves throughout the known extent of $\mathrm{H}$ I, with lower average UV surface brightness in the XUV-disk. Nevertheless, localized UV-bright clumps are obvious even in the most extended parts of the $\mathrm{H}$ I disk. As expected, patterns in the distribution of XUV complexes are tied to arm features in the H I morphology of NGC 3198. We can discern SDSS detections for a limited number of the brightest inner XUV-disk UV complexes. NGC 3198 is the brightest member of group containing five galaxies, including NGC 3319 (a Type 2 XUV-disk). However, computation of a tidal perturbation parameter (see $\S 3.3 .3$ ) indicates that NGC 3198 is effectively isolated in terms of tidal interaction.

NGC 3344 (Fig. 16.14).--This (R)SAB(r)bc H II galaxy has a large UV disk which gradually fades with radius (similar to NGC 628, but less obvious). Both Verdes-Montenegro et al. (2000) and Corbelli et al. (1989) present H I maps of NGC 3344. UV emission is smoothly extended but bright, even if not so in the optical. NGC 3344 has two companions (UGC 05672 and NGC 3274, altogether constituting NOGG 468), although is not currently tidally interacting (§ 3.3.3). SDSS imaging helps to differentiate the outermost clumps from background galaxies.

NGC 3359 (Fig. 16.15).-A two-arm SB(rs)c H II galaxy with XUV-disk complexes distributed in long, wrapping spiral features beginning at the ends of the major axis where the optical spiral arms cease. The eastern XUV arm is of higher surface brightness near 
the inner disk, whereas the western XUV arm is more prominent at large galactocentric distances. Nevertheless, both UV-bright complexes in both arms can be seen out to nearly 3 times $R_{25}$. NGC 3359 has been categorized as an "isolated" galaxy in several studies (Karachentseva 1973; Varela et al. 2004; Giuricin et al. 2000; Verdes-Montenegro et al. 2005), although it is known to have a low-mass, LSB object detected in H I (Ball 1986) and Palomar plates. This object is $15^{\prime}$ southwest of the galaxy, beyond the limits of the multiwavelength images we present. However, our GALEX data do recover the Ball (1986) LSB object as a resolved FUV-bright clump. The H I structure of NGC 3359 and this LSB companion has also been studied by Broeils (1992) and with new WSRT data in van der Hulst \& Sancisi (2005, who measure an $\mathrm{H}$ I mass of $1.8 \times 10^{8} M_{\odot}$ for the companion). The van der Hulst \& Sancisi observations are sensitive enough to discern a connection between the outer LSB cloud and NGC 3359's spiral structure, meaning our UV detection of the object makes NGC 3359 among the most extended UV disks known (relative to optical size). SDSS observations are sensitive to only the brightest of the XUV complexes.

NGC 3621 (Fig. 16.16). - This SA(s)d galaxy is of very large angular size in the UV, at least partially owing to its proximity $(8.3 \mathrm{Mpc})$. GALEX imaging reveals SF occurring throughout almost all of the known H I extent $\left(32^{\prime} \times 11^{\prime}\right.$; Koribalski et al. 2004 ; THINGS). A linear feature running along the major axis in $\mathrm{H}$ i maps could be the remnants of a previous interaction, or even a dwarf currently being accreted (see the LVHIS map of W. Walsh, especially velocity dispersion). The XUV-disk has a high surface covering factor in the UV and can be traced as a LSB optical structure as well. Spiral structure is evident throughout the XUV-disk. An abrupt drop in the radial UV surface brightness profile (Gil de Paz et al. 2007a) indicates the transition to the LSB outer disk. Incidentally, this transition occurs within the $D_{25}$ extent of the galaxy, despite the continuation of LSB features to more extreme galactocentric distance. The NOG catalog indicates that NGC 3621 is isolated, although the linear feature in the $\mathrm{H}$ I maps suggests it has not always been so. Perhaps the elevated degree to which $\mathrm{SF}$ is now occurring in the outer $\mathrm{H}$ I disk was stimulated by this interaction. If so, the age of the interaction could be inferred from the mean age of the LSB stellar population.

NGC 3705 (Fig. 16.17).-Belonging to the Leo I group, this SAB(r)ab; LINER H II galaxy has faint discrete XUV complexes east and west of the disk superimposed on a more diffuse component in our NUV-only GALEX imagery, although it is difficult to "confirm" even with SDSS images. Although it lives in a group environment, NGC 3705 is not currently interacting according to the tidal perturbation parameter. This is a somewhat marginal XUV-disk, as the UV structure is limited in nature.

$N G C 4254$ [M 99] (Fig. 16.18). - This Virgo cluster member, an SA(s)c galaxy, exhibits peculiar lopsided XUV emission in our deep NUV GALEX imaging. The UV clumps are located to the southwest of the bright inner disk over an azimuthal range of about $45^{\circ}$ and extending to approximately 2 times the $D_{25}$ radius. The XUV structure, although of a high surface covering factor, does not appear to be related to the spiral structure of the inner disk. The XUV disk of NGC 4254 is also odd because the $\mathrm{H}$ I disk of the galaxy is slightly extended in an opposite direction, to the north of the disk (VIVA of Kenney et al. 1995; Braun et al. 2007). Furthermore, ALFALFA observations have demonstrated that NGC 4254 has a very low column density H i stream (see the Giovanelli ALFALFA follow-up proposal) running $\sim 30^{\prime}$ north-northwest of the galaxy and including VIRGO HI21 (Minchin et al. 2005). SDSS imaging shows little in the vicinity of the lopsided XUV-disk.

NGC 4258 [M 106] (Fig. 16.19).- GALEX observations of this SAB(s)bc; LINER Seyfert 1.9 galaxy indicate a very large extent $\left(\sim 25^{\prime}\right)$, although the substantial inclination of the disk (and the orientation of its warped outer $\mathrm{H}_{\mathrm{I}}$ ) make it difficult to say precisely to what galactocentric distance XUV features are seen. The most significant area with XUV-disk clumps is south of the disk. SDSS data detect very few of these star clusters. NGC 4258 is the brightest galaxy in a group having over 20 members, possibly interacting with NGC $4248(f=-2.2)$.

$N G C 4383$ (Fig. 16.20).- This Sa? pec H II galaxy, found in the outskirts of the Virgo cluster, has a very bright inner disk, centered within an S-shaped, spiral pattern of intermediate surface brightness UV clumps. This spiral morphology is unapparent in visible light imagery, with some authors calling the galaxy amorphous. UV clumps are distributed throughout the extent of the $\mathrm{H}$ I mapped by Kenney et al. (VIVA) - most notably to the south-southeast, east, and northeast of the galaxy. The H $\alpha$ emission of NGC 4383 shows some $\mathrm{H}$ in regions associated with the extended UV clumps (Gavazzi et al. 2003, 2006) and an inner bright, biconical filamentary structure (Koopman \& Kenney 2006). SDSS imagery reveals only the blue clumps composing the innermost northern part of the S-shaped spiral pattern. NGC 4383 has an overall appearance similar to NGC 1140, although they are in different XUV classes. This illustrates the difficulty of classifying objects having such complex structure.

$N G C 4414$ (Fig. 16.21).-A member of the Coma I group (dominated by NGC 4631), our observations of this SA(rs)c? LINER galaxy reveal a bright main disk surrounded by XUV clumps distributed in disorganized (tightly wrapped?) spiral features at almost all position angles. The clumps are not located at extreme galactocentric distances, but rather tend to hug the main disk. This galaxy is one of the few to have confirmed outer disk molecular clouds (Braine \& Herpin 2004) at a limited number of positions near $\mathrm{H}$ I peaks (Thornley \& Mundy 1997). Deep H $\alpha$ imaging reveals sporadic H II regions coinciding with some of the XUV clumps (Gavazzi et al. 2003, 2006). SDSS imagery allows one to see the brightest XUV-disk stellar complexes. Despite belonging to the Coma I group, NGC 4414 is dynamically isolated in the sense that it has no known tidally perturbing companion.

NGC 4438 (Fig. 16.22).- This SA(s)0/a pec: LINER galaxy is interacting with NGC 4435 as they cross the center of the Virgo Cluster at high speed (Vollmer et al. 2005). The high-speed collision is discussed by Kenney et al. (1995). GALEX observations analyzed by Boselli et al. (2005) show the presence of very recent star formation in a tidal tail extending to the northwest of the galaxy; this feature was undetected at other wavelengths (including $R$ down to $27.8 \mathrm{mag} \mathrm{arcsec}^{-2}$ via Subaru). The UV-visible-NIR SED fitting of Boselli et al. suggests that the burst of star formation is notably younger than the dynamical age of the NGC 4438/NGC 4435 interaction. SDSS imagery highlights a large dust concentration on the west side of NGC 4438. It is interesting to note that the H i streamer of nearby NGC 4388 (Oosterloo \& van Gorkom 2005) points very near to NGC 4438's northwest XUV feature, with similar orientation.

NGC 4559 (Fig. 16.23).—GALEX observations of this SA(r)0+; H II LINER galaxy show diffuse off-plane FUV emission perhaps associated with extraplanar $\mathrm{H}$ i of Barbieri et al. (2005). Discrete XUV-disk complexes are arranged in spiral arms tightly wrapping the on galaxy periphery in a manner similar to NGC 4414. SDSS imaging at blue wavelengths detects some of the UV-bright clumps. NGC 4559 is a Coma I group member. 
NGC 4625 (Fig. 16.24).- One of the first known XUV-disks, GALEX observations of this $\mathrm{SAB}(\mathrm{rs}) \mathrm{m}$ pec galaxy show that the outer disk is organized into spiral arms, although the surface covering factor remains quite high compared to M83 or NGC 5055, for instance, which both have a more filamentary appearance. Abundance is low (although not primordial) in the outer disk, as measured in a population of faint $\mathrm{H}$ il regions (Gil de Paz et al. 2007b). Most of the XUV-disk stellar complexes are too faint for individual detection by SDSS. NGC 4625 is interacting with NGC 4618 and possibly NGC 4625A (Gil de Paz et al. 2005). NGC 4625 is a member of the NGC 4631 group. H I data for the NGC 4625/4618 pair were obtained by the WHISP project and show the usual correlation between $\mathrm{H}$ I substructure and the outer disk UV clumps.

NGC 5055 [M63] (Fig. 16.25).- This is an SA(rs)bc H II / LINER galaxy with a very large warped H i disk (Battaglia et al. 2006). GALEX shows that the outer $\mathrm{H}$ I disk has recently formed massive stars, principally along ridges in the gas distribution. One particular region of UV emission to the southwest of the galaxy is possibly a TDG (namely UGCA 342; Bremnes et al. [1999] suggest it is a "condensation" in the outer gas disk). NGC 5055 is a member of the M51 group (7 members). NGC 5055 appears to be interacting with UGC 08313, found to the northwest and connected by a filament of $\mathrm{H}$ I containing UV-bright complexes. SDSS imaging recovers UGCA 342 and a few other XUV-clumps.

NGC 5236 [M83] (Fig. 16.26). - The prototypical XUV-disk galaxy [SAB(s)c; H II starburst] exhibits clumpy SF complexes associated with local maxima throughout an extensive, filamentary H I disk (Thilker et al. 2005a). The distribution of UV complexes is sparse, with a low surface covering factor, although there are many sites of recent star formation. Some of the XUV complexes do contain faint $\mathrm{H}$ II regions, which have low abundance $\left(\sim Z_{\odot} / 10\right)$ and are often ionized by single massive stars (Gil de Paz et al. 2007b). Past interaction with other members of the Centaurus A group, including NGC 5253 and KK 208 (Karachentseva \& Karachentsev 1998), is considered likely. More details on NGC 5236 are presented by Thilker et al. (2005a) and Gil de Paz et al. (2007b).

NGC 5457 [M101] (Fig. 16.27).- This giant SAB(rs)cd galaxy dominates a group having at least 8 members, including NGC 5474 and NGC 5477, with both of which it is probably interacting. Our GALEX observations show a radial gradient in FUV - NUV (Gil de Paz et al. 2007a), getting clearly bluer at large galactocentric distances. Although discrete SF complexes do appear redder in the inner disk, some of the central NUV flux originates in a diffuse slightly older stellar population. This component gradually fades, leaving only the very blue, prominent branching spiral arms at large galactocentric distance. Comparison with SDSS imagery confirms that a wealth of filamentary UV-bright cluster complexes in the outermost disk can be traced easily in the GALEX observation (Bianchi et al. 2005), but only intermittently at optical wavelengths. The previously unrecognized extent of recent star formation is greatest on the east side of the galaxy, reaching most of the way to NGC 5477. Prior UV observations of Waller et al. (1997) suggested that tidal interaction with companions and an internal perturber (NGC 5471) have produced the kinked, linear morphology spiral arms in M101. Low metallicity of the outer disk has been demonstrated by Garnett \& Kennicutt (1994) for the H II region H681. The H I distribution (Beale \& Davies 1969; Rogstad \& Shostak 1971; van der Hulst \& Sancisi 1988; Braun 1995) is lopsided and conceivably distorted by tidal forces.

$N G C 6239$ (Fig. 16.28).-GALEX observations of this $\mathrm{SB}(\mathrm{s}) \mathrm{b}$ pec? H II galaxy show a pair of filaments stretching northwest of the galaxy and a looplike structure to the southeast, both highlighted in the UV. SDSS imaging reveals only the innermost portions of the northwest filaments. $\mathrm{H} \alpha$ observations of Garcia-Barreto et al. (1996) show only very limited line emission from beyond the inner disk. The H I study of Hogg \& Roberts (2001) show that NGC 6239 is interacting with a small companion to the east (SDSS J165045.93+ 424320.4), even though it is isolated according to both the NOG catalog and our tidal perturbation parameter analysis.

UGC 10791 (Fig. 16.29).- - This Sm: galaxy has extended morphology in the form of scattered UV-bright clumps, arranged with no discernible resemblance of spiral arms. H I observations are available through the WHISP project (van der Hulst et al. 2001; van der Hulst 2002; Swaters et al. 2002; Noordermeer et al. 2005). UGC 10791 does not belong to a NOG group (it was too faint to be considered by Giuricin et al. 2000), but is possibly interacting with NGC 6340 .

NGC 6902 (Fig. 16.30). - Optical imaging of this SA(r)b LINER shows a LSB disk component with tightly wrapped spiral structure outside of a bright inner region, which hosts a ring and bar. GALEX reveals that this faint outer disk is actively forming stars, even at locations where the LSB optical disk is not apparent. The UV disk fades away in a ragged, but continuous manner. This relatively understudied galaxy (considering its angular size) was considered in detail by Gallagher (1979). NGC 6902 is the brightest member of a triplet, also containing NGC 6902B and IC 4946, but is only weakly interacting (if at all).

\section{A2. MIXED-TYPE XUV-DISKS}

On occasion a galaxy may satisfy both XUV-disk classification criteria, exhibiting a large, blue LSB zone and structured UV-bright complexes at extreme galactocentric distances beyond the traditional SF threshold. We call such objects mixed-type XUV-disks. They represent a population of galaxies undergoing significant structural change, like Type 2 XUV-disks, but they most likely have a comparatively extended gas distribution in addition.

NGC 1051 [NGC 0961] (Fig. 16.31). - This SB(rs)m pec galaxy exhibits flocculent, clumpy XUV disk structure, extending to about $3.5^{\prime}$. In contrast, the $K_{s}$-band emission is compact, thereby qualifying the galaxy also as a Type 2 XUV disk. NGC 1051 is a member of the NGC 988 group (see entry for NGC 1042). SDSS imagery shows LSB emission over some of NGC 1051 's XUV disk, particularly to the northeast.

NGC 2403 (Fig. 16.32).- - Often compared to M33, this SAB(s)cd H II galaxy satisfies both our criteria for being considered an XUVdisk. The outermost XUV disk complexes lie near local maxima within the $\mathrm{H}$ I filament, which begins near the northwest end of the major axis and wraps counterclockwise around the north side of the galaxy (Fraternali et al. 2002). In at least one case, there appears to be an $\mathrm{H}$ I shell surrounding the XUV complex (at $07^{\mathrm{h}} 34^{\mathrm{m}} 47.5^{\mathrm{s}},+65^{\circ} 41^{\prime} 29^{\prime \prime}$ ), highlighting the feedback of outer disk star formation on the gas morphology. The combination of NGC 2403's large angular extent (projecting background galaxies onto the XUV disk) and proximity (breaking up complexes) makes it difficult to tell the difference between isolated XUV clumps and background sources seen through the most rarified portions of the $\mathrm{H}$ I disk. Gas accretion has been suggested as the origin of extraplanar $\mathrm{H}$ I (Fraternali \& Binney 2006) in this object. Known or likely companions include NGC 2366 (NOG catalog), DDO 44 (KK61), Holmberg II, UGC 04483, and K52 (Karachentsev 1994; Karachentsev et al. 2000). NGC 2403 may be an outlier galaxy within the M81 group. 
UGC 04393 (Fig. 16.33).-Our GALEX observations of this SBc? H II galaxy show it to have an asymmetric distribution of star formation activity, suggesting interaction with an unidentified companion. In particular, the inner disk has a spiral-like arc of UV-bright emission surrounding it to the southeast. This feature, however, is contained within the body of $\mu_{\mathrm{NUV}}=27.35$, which does not place it in our XUV class, despite the peculiar morphology. SDSS imagery of UGC 04393 is peculiar in the sense that the distribution of blue starforming clumps appears projected against an underlying redder stellar population without morphological correspondence. Haynes et al. (1998) show an H i profile with no notable velocity asymmetry in this seemingly isolated galaxy. Thus, our suggestion of interaction must remain unconfirmed at present. The most extreme galactocentric distance GALEX detection is a collection of very faint clumps to the northwest of the galaxy, which may be a continuation of the inner spiral feature. In addition, a possible curved filament of faint UV emission is found just beyond the northeast end of the major axis.

NGC 5474 (Fig. 16.34).- This companion of M101 (NGC 5457) is well known optically for hosting an off-center nucleus, most likely indicative of prior interaction (with M101?). Imaging from GALEX shows that scattered SF complexes exist in the outer disk of this SA(s)cd pec H II galaxy, most especially to the west and southeast. NGC 5474 is a galaxy in which the distribution of UV-bright sources falls off smoothly with galactocentric distance, even if extended. The H i structure of NGC 5474 was studied in Rownd et al. (1994), who noted the clumpy nature of the gas distribution without any clear evidence for spiral features. At a limit of $\sim 2 \times 10^{20} \mathrm{~cm}^{-2}$, the $\mathrm{H}$ I disk covers an area of $9^{\prime} \times 7^{\prime}$. Curiously, the GALEX data show spiral arm structure, as do H $\alpha$ observations (Hodge \& Kennicutt 1983). Moreover, these features begin at the position of the offset nucleus, rather than the center of the overall optical distribution. SDSS data again suggest a dramatic disconnection between the stars contributing to the visible light morphology and the (spiral) pattern of recent star birth, similar to what we found for UGC 04393.

NGC 5832 (Fig. 16.35).- NUV-only GALEX observations of this SB(rs)b? galaxy reveal faint XUV emission primarily in the southwest quadrant of the galaxy. There does not appear to be coherent spiral morphology in the outer disk. Kazarian (1996) noted that NGC 5832 belongs to a class of galaxies having UV excess. This galaxy is isolated according to both the NOG catalog of groups and the tidal perturbation parameter (no possible perturbers).

UGC 10445 (Fig. 16.36).- GALEX observations of UGC 10445 present an outer disk with numerous clumps and diffuse emission, jointly resulting in a fairly high surface covering factor. The distribution of XUV emission is lopsided in the sense that there is very little extended UV on the southwest side of the galaxy. H $\alpha$ imaging of this isolated SBc starburst galaxy (van Zee 2001) shows relatively few H II regions in outer UV area. This starbursting dwarf was also studied with Spitzer by Hinz et al. (2006), who present evidence for an extended dust distribution. We differ slightly regarding the Hinz et al. interpretation of the GALEX imagery. They contend that the dust distribution is more extended than UV, but this appears to be due to the smoothing scale used for the GALEX data. H i morphology (available through WHISP; van der Hulst et al. 2001; van der Hulst 2002; Swaters et al. 2002; Noordermeer et al. 2005) is unremarkable, although the XUV-disk and gas appear roughly coextensive. Some of the XUV stellar complexes are visible in the blue SDSS bands, especially at modest galactocentric distance in the LSB zone.

$N G C 7418$ A (Fig. 16.37). - This isolated SA(rs)d: galaxy lies about $15.5^{\prime}$ from NGC 7418, but at a velocity separation $>600 \mathrm{~km} \mathrm{~s}{ }^{-1}$. Our GALEX data show a two-armed feathery-looking, spiral pattern of UV-bright clumps stretching from the northeast to southwest. Within a radius of about $1^{\prime}$ the UV disk takes on a more filled appearance, and a LSB optical component of the disk starts to be visible. The nucleus is bright and compact at UV and optical wavelengths. The brightest of NGC 7418A's XUV complexes can be discerned in DSS2-red plates.

\section{A3. TYPE 2 XUV-DISKS}

NGC 991 (Fig. 16.38).- - Our GALEX observations of this SAB(rs)c galaxy show fairly sharp, outer UV edge to the well-defined pattern of spiral arms, in contrast to the Type 1 XUV-disks. In fact, the general UV morphology is quite similar to the appearance of the galaxy in SDSS imaging at $u, g$. The distribution of H I has been studied by Kornreich et al. (2000). Their data, in combination with our UV imagery, suggest that recent SF in NGC 991 is confined to areas with $N(\mathrm{H} \mathrm{I})>$ few $\times 10^{20} \mathrm{~cm}^{-2}$. NGC 991 belongs to a group of 11 objects dominated by NGC 988, but is dynamically unperturbed at present.

$N G C 1140$ (Fig. 16.39)._-GALEX imaging shows additional structure in this IBm pec:; H II Seyfert 2 galaxy, unrecognized at optical wavelengths, despite its classification as a Type 2 XUV-disk. The UV-optical morphology is complex, with a curved (out-of-plane?), clumpy feature oriented to the southwest visible in both the GALEX and DSS images, plus a set of UV-only (not seen in the DSS) sources extending north of the galaxy center for nearly $2^{\prime}$. The inner disk has a diffuse-looking background of NUV bright emission, with very bright central surface brightness at the nucleus. The peculiar UV-optical morphology of NGC 1140 is suggestive of interaction, although the galaxy is considered isolated in the NOG catalog. As noted earlier, NGC 4383 and NGC 1140 are similar objects, even though our classification criteria put them into different XUV classes.

$N G C 2090$ (Fig. 16.40). - Our GALEX imaging of this isolated SA:(rs)b galaxy reveals a LSB outer disk barely detected in DSS2-red plates and not at all in the NIR $\left(2 \mathrm{MASS}-K_{S}\right)$, but prominent at UV wavelengths. This object is a prototypical example of a disk in formation at the present epoch. The GALEX data show a redder inner disk, surrounded by a much bluer, flocculent outer region characterized by discontinuous spiral segments. H il regions are found in the Type 2 XUV-disk of NGC 2090 (Koopmann \& Kenney 2006 ). In the bright inner part of the galaxy, some of these $\mathrm{H}$ II regions (and associated UV emission) take the form of a ring structure.

ESO 556-012 (Fig. 16.41). - This SB(s)m galaxy appears unremarkable in our GALEX NUV imagery or optical plates, but comparison with 2MASS imagery shows that almost all of the UV disk is extremely faint at $K_{s}$-band. Little more than the nucleus is detectable in the NIR. The GALEX data show a spiral arm wrapping around the disk to north side.

$N G C 2541$ (Fig. 16.42). - The conspicuous outer spiral structure of this SA(s)cd LINER galaxy detected in our GALEX observations is not seen at all in the 2 MASS- $K_{S}$ imagery, and only partially in visible light. SDSS imaging shows a few faint sources in the XUV-disk LSB zone. H I observations from WHISP and Broeils \& van Woerden (1994) show that the galaxy has an extended gas distribution. NGC 2541 is the brightest galaxy in a small group (of 3+?), but isolated according to tidal perturbation parameter analysis.

UGC 04390 (Fig. 16.43).-GALEX imaging of this SBd galaxy shows a clumpy UV disk which begins crowded at small galactocentric distances but becomes progressively more sparse in the outer region. The UV disk is notably lopsided, with greater extension to 
the east. Such asymmetry is not as evident in the DSS2-red image. In the 2MASS- $K_{S}$ data, essentially only the nucleus and bar are detected.

$U G C 04800$ (Fig. 16.44).- Our GALEX data for this inclined, isolated SB(s)cd? galaxy show a bright UV disk with a sharp outer edge. The disk is also bright in SDSS imaging, but it has a small $K_{s}$-band size in comparison to GALEX-SDSS extent.

IC 2574 (Fig. 16.45).- This SAB(s)m member of the M 81 group presents a dramatic integral-shaped spiral morphology in our GALEX imaging, which cannot be seen in the DSS2-red plates. At the $K_{s}$-band the galaxy is only detected as a fuzzy patch of emission. IC 2574 has received attention, even in the UV, for the presence of a supergiant $(\mathrm{H}$ I) shell in the northern part of the galaxy (Cannon et al. 2005; Walter 2001; Stewart \& Walter 2000; Walter \& Brinks 1999).

NGC 3319 (Fig. 16.46).- GALEX observations of this SB(rs)cd H II: galaxy, belonging to the NGC 3198 group of at least 5 optically bright galaxies, show a highly filled UV disk with prominent spiral arms beginning at the end of the bar structure. SDSS imaging shows the brightest parts of the spiral arms in the form of a string of blue clumps with some more diffuse underlying emission at redder wavelengths. The H I distribution of NGC 3319 was studied by Moore \& Gottesman (1998) using the VLA. They detected a gas-rich companion lying $11^{\prime}$ south of NGC 3319 and an $\mathrm{H}$ I tail (also to the south), which they postulate was created by an interaction of the two galaxies.

NGC 4116 (Fig. 16.47).- - Our GALEX imaging of this $\mathrm{SB}(\mathrm{rs})$ dm galaxy paired with NGC 4123 demonstrate that the UV disk is highly structured into spiral segments but lacks symmetry. This disturbed structure is also visible in SDSS images, but the GALEX sensitivity reveals more morphological features in the outer disk. For example, our NUV image shows a tightly wrapped spiral arm encircling the south end of the major axis. As is the case for many of our Type 2 XUV-disks, the 2 MASS- $K_{s}$ data reveal essentially just the innermost portion of the galaxy (nucleus and bar). Both NGC 4116 and NGC 4123 are part of a quartet dominated by NGC 4179, but it is not certain that NGC 4116 is interacting with any galaxy (even NGC 4123).

$N G C 4236$ (Fig. 16.48).- - This SB(s)dm galaxy has very large angular extent $\left(>20^{\prime}\right)$ in our GALEX survey images. Spiral structure accentuated by large clumpy complexes is apparent in the UV. Much of the outer UV spiral is barely discernible on the DSS2-red imagery. The VLA H I observations of Braun (1995) show that the gas distribution has a highly structured morphology even in the outer disk, matching the UV emission quite closely. NGC 4236 lies in a grouping with UGC 08201, and is often associated with the M81 group. Holm 357b also lies to the northeast of NGC 4236, at unknown redshift.

UGC 08365 (Fig. 16.49).- GALEX imagery of this $\mathrm{SB}(\mathrm{s}) \mathrm{d}$ galaxy shows a disk characterized by scattered UV-bright clumps. The highest surface brightness portion of the UV morphology is elongated in nearly the same direction as the bar visible at optical (SDSS) wavelengths. The $K_{s}$-band data from 2MASS only faintly reveal the bar, and nothing of the remaining disk. UGC 08365 belongs to a group of three objects dominated by NGC 5013.

NGC 5705 (Fig. 16.50).- Our UV imagery of this SB(rs)d galaxy in a large group of $25+$ galaxies (brightest member NGC 5746) shows loose spiral structure composed of numerous bright clumps with underlying diffuse emission. SDSS imagery shows that at least some of these clumps are massive enough to be detected optically. NGC 5705 may have close companions (SHOC 476, 2QZ J143953.9004318, and SDSS J143956.60-004439.3).

NGC 5727 (Fig. 16.51).- This UV-bright, optically faint SABdm galaxy has blue UV-optical color over almost the entire disk, with only slightly redder colors toward the center, as illustrated by SDSS imaging. Pisano et al. (2002) indicate that it has a companion to the northwest, which they name NGC 5727A.

NGC 6255 (Fig. 16.52).- GALEX and SDSS observations of this SBcd: galaxy show that the disk has well-defined, blue spiral arms on the north and south sides of the target. NGC 6255 belongs to a triplet of bright galaxies, dominated by NGC 6207. The galaxy has been noted to have a possible companion (HS 1653+3634) which lies $75^{\prime \prime}$ to the east. It seems more likely from GALEX data that this object is just a particularly bright, blue cluster complex in the XUV-disk of NGC 6255.

ESO 406-042 (Fig. 16.53). - GALEX observations of this comparatively isolated SAB(s)m galaxy show a clumpy structure with little organization into spiral features. The disk (even the center) is almost undetected by 2MASS.

ESO 407-014 (Fig. 16.54). - Our GALEX imaging of this isolated SB(s)c? H II galaxy show obvious spiral structure in an outer disk which has surface brightness at visible wavelengths distinctly fainter than the inner galaxy. In this manner it resembles NGC 2090. Indeed, the disk has a bright central $2 \mathrm{MASS}-K_{s}$ nuclear region with a seemingly sharp edge.

\section{APPENDIX B}

\section{INSIDE-OUT DISK FORMATION MODELS}

As mentioned in $\S 3.1 .2$, we compared measured characteristics of the LSB zone against predictions derived from the linear insideout disk formation models of Muńoz-Mateos et al. (2007). In particular, the models were used to estimate the anticipated locus of "normal" galaxies growing from the inside-out in Figure 2, which plots the relative area of the LSB zone versus GALEX-2MASS color measured in the LSB zone $\left[S(\mathrm{LSB}) / S\left(K_{80}\right)\right.$ vs. UV(AB) $\left.-K_{s}(\mathrm{AB})\right]$. Details of the modeling are given in Muńoz-Mateos et al. (2007). Here we describe the most important points and any modifications for our current purpose.

We assume that the SFR surface density of a disk can be parameterized as $\Sigma_{\mathrm{SFR}}(r, t) \sim \exp (-t / \tau) \exp \left[-r /\left(a_{0}+b t\right)\right]$. The first term imposes an exponential SFH with a timescale $\tau$, and whereas the second term introduces the radial exponential profile, whose scale length is supposed to vary linearly with time. Here $a_{0}$ is the initial size (in kpc) at the redshift where the thin disk is formed $(z \sim 1-2)$, and $b$ is the growth rate of the scale length (kpc/Gyr). By integrating $\Sigma_{\mathrm{SFR}}(r, t)$ since $z \sim 1-2$ until $z=0$, the model computes the current stellar mass surface density profile for a grid of values of $a_{0}$ and $b$. We assume that the fraction of recycled gas is $R=0.3$, for a typical IMF. We also feed the model with the central SFR surface densities of the disks observed today, in order to properly normalize $\Sigma_{\text {SFR }}(r, t)$. Finally, an average internal extinction of 1 mag in the FUV is used both when locating the UV and $K_{80}$ contours and when measuring the colors. 
The original models of Muńoz-Mateos et al. (2007) did not explicitly take into account the formation and development of the bulge. Since the bulge is necessary to delineate the LSB zone in the same way we did observationally, we implemented a bulge component assuming a de Vaucouleurs profile, with a given effective surface brightness $\mu_{e}$. (The effective radius is not needed, since it can be internally computed from assumed bulge-to-disk ratio, $\mu_{e}$, and the predicted disk luminosity.) It seems that the bulge is necessary to determine where to measure, but the measurements themselves depend more on the properties of the disk generated by the model.

Aguerri, J. A. L., Elias-Rosa, N., Corsini, E. M., \& Muñoz-Tuñón, C. 2005 , A\&A, 434, 109

Allen, R. J., Atherton, P. D., \& Tilanus, R. P. J. 1986, Nature, 319, 296

Auld, R., et al. 2006, MNRAS, 371, 1617

Ball, R. 1986, ApJ, 307, 453

Barbieri, C. V., Fraternali, F., Oosterloo, T., Bertin, G., Boomsma, R., \& Sancisi, R. 2005, A\&A, 439, 947

Barth, A. J. 2007, AJ, 133, 1085

Battaglia, G., Fraternali, F., Oosterloo, T., \& Sancisi, R. 2006, A\&A, 447, 49

Beale, J. S., \& Davies, R. D. 1969, Nature, 221, 531

Beckman, J. E., Casuso, E., Zurita, A., \& Relaño, M. 2004, in Penetrating Bars through Masks of Cosmic Dust (Dordrecht: Kluwer), 119

Bekki, K., \& Chiba, M. 2006, ApJ, 637, L97

Bell, E. F., \& de Jong, R. S. 2001, ApJ, 550, 212

Bianchi, L., et al. 2003, in The Local Group as an Astrophysical Laboratory, ed.

M. Livio \& T. Brown (Baltimore: STScI), 10 2005, ApJ, 619, L71

2007, in IAU Symp. 235, Galaxy Evolution across the Hubble Time, ed. F. Combes \& J. Palous (Cambridge: Cambridge Univ. Press), in press

Bland-Hawthorn, J., Vlajić, M., Freeman, K. C., \& Draine, B. T. 2005, ApJ, 629,239

Boissier, S., Boselli, A., Prantzos, N., \& Gavazzi, G. 2001, MNRAS, 321, 733

Boissier, S., \& Prantzos, N. 1999, MNRAS, 307, 857 2000, MNRAS, 312, 398

Boissier, S., et al. 2007, ApJS, 173, 524

Bonnell, I. A. 2000, Star Formation from the Small to the Large Scale, ed. F. Favata, A. Kaas, \& A. Wilson (ESA SP 445; Noordwijk: ESA), 273

Boselli, A., Boissier, S., Cortese, L., Gil de Paz, A., Seibert, M., Madore, B. F., Buat, V., \& Martin, D. C. 2006, ApJ, 651, 811

Boselli, A., \& Gavazzi, G. 2006, PASP, 118, 517

Boselli, A., Gavazzi, G., Donas, J., \& Scodeggio, M. 2001, AJ, 121, 753

Boselli, A., et al. 2005, ApJ, 623, L13

Bosma, A. 1981, AJ, 86, 1791

Bournaud, F., Combes, F., Jog, C. J., \& Puerari, I. 2005, A\&A, 438, 507

Boyce, P. J., et al. 2001, ApJ, 560, L127

Braine, J., Duc, P.-A., Lisenfeld, U., Charmandaris, V., Vallejo, O., Leon, S., \& Brinks, E. 2001, A\&A, 378, 51

Braine, J., \& Herpin, F. 2004, Nature, 432, 369

Braun, R. 1995, A\&AS, 114, 409 1997, ApJ, 484, 637

Braun, R., Oosterloo, T. A., Morganti, R., Klein, U., \& Beck, R. 2007, A\&A, 461,455

Braun, R., \& Thilker, D. A. 2004, A\&A, 417, 421

Bremnes, T., Binggeli, B., \& Prugniel, P. 1999, A\&AS, 137, 337

Briggs, F. H. 1986, ApJ, 300, 613

Broeils, A. H. 1992, Ph.D. thesis, Univ. of Groningen

Broeils, A. H., \& van Woerden, H. 1994, A\&AS, 107, 129

Brook, C. B., Kawata, D., Martel, H., Gibson, B. K., \& Bailin, J. 2006, ApJ, 639,126

Brouillet, N., Henkel, C., \& Baudry, A. 1992, A\&A, 262, L5

Brown, T. M., Smith, E., Ferguson, H. C., Rich, R. M., Guhathakurta, P., Renzini, A., Sweigart, A. V., \& Kimble, R. A. 2006, ApJ, 652, 323

Bruzual, G., \& Charlot, S. 2003, MNRAS, 344, 1000

Byrd, G. G., \& Howard, S. 1992, AJ, 103, 1089

Cannon, J. M., et al. 2005, ApJ, 630, L37

Chabrier, G. 2003, PASP, 115, 763

Chiappini, C., Romano, D., \& Matteucci, F. 2003, MNRAS, 339, 63

Corbelli, E., Schneider, S. E., \& Salpeter, E. E. 1989, AJ, 97, 390

Cortese, L., et al. 2006, ApJ, 637, 242

Crowl, H. H., \& Kenney, J. D. P. 2006, ApJ, 649, L75

Crowl, H. H., Kenney, J. D., van Gorkom, J. H., Chung, A., \& Rose, J. A. 2006, BAAS, 209, 211.11

Dahari, O. 1984, AJ, 89, 966

Dale, D. A., et al. 2007, ApJ, 655, 863

de Blok, W. J. G., \& Walter, F. 2006, AJ, 131, 363

de Jong, R. S.. 1996, A\&A, 313, 377

Elmegreen, B. G.. 2006, ApJ, 648, 572

Elmegreen, B. G., \& Hunter, D. A. 2006, ApJ, 636, 712

Elmegreen, B. G., \& Parravano A. 1994, ApJ, 435, L121

\section{RERENCES}

Elmegreen, B. G., \& Scalo, J. 2006, ApJ, 636, 149

Erwin, P., Beckman, J. E., \& Pohlen, M. 2005, ApJ, 626, L81

Ferguson, A. M. N. 1998, Ph.D. thesis, Johns Hopkins University

Ferguson, A. M. N., Gallagher, J. S., \& Wyse, R. F. G. 1998a, AJ, 116, 673

Ferguson, A. M. N., Wyse, R. F. G., Gallagher, J. S., \& Hunter, D. A. 1998b, ApJ, 506, L19

Florido, E., Battaner, E., Guijarro, A., Garzón, F., \& Jiménez-Vicente, J. 2001, A\&A, 378, 82

Flynn, L., Walterbos, R. A. M., Thilker, D. A., \& Fierro, V. 1998, BAAS, 30, 1352

Fraternali, F., \& Binney, J. J. 2006, MNRAS, 366, 449

Fraternali, F., van Moorsel, G., Sancisi, R., \& Oosterloo, T. 2002, AJ, 123, 3124

Gallagher, J. S. 1979, AJ, 84, 1281

Galletta, G., Rodighiero, G., Bettoni, D., Moles, M., \& Varela, J. 2006, A\&A, 456, 91

García-Barreto, J. A., Franco, J., Carrillo, R., Venegas, S., \& Escalante-Ramírez, B. 1996, Rev. Mex. AA, 32, 89

Garnett, D. R., \& Kennicutt, R. C., Jr. 1994, ApJ, 426, 123

Gavazzi, G., et al. 1996, A\&A, 312, 397

2003, A\&A, 400, 451

2006, A\&A, 446, 839

Gil de Paz, A., et al. 2005, ApJ, 627, L29

2007a, ApJS, 173, 185

2007b, ApJ, 661, 115

Giuricin, G., Marinoni, C., Ceriani, L., \& Pisani, A. 2000, ApJ, 543, 178

Greenawalt, B. 1998, Ph.D. thesis, New Mexico State Univ.

Gutiérrez, C. M., Alonso, M. S., Funes, J. G., \& Ribeiro, M. B. 2006, AJ, 132, 596

Hawarden, T. G., van Woerden, H., Goss, W. M., Mebold, U., \& Peterson, B. A. 1979, A\&A, 76, 230

Haynes, M. P., van Zee, L., Hogg, D. E., Roberts, M. S., \& Maddalena, R. J. 1998, AJ, 115, 62

Heller, A., Almoznino, E., \& Brosch, N. 1999, MNRAS, 304, 8

Henry, R. B. C., \& Worthey, G. 1999, PASP, 111, 919

Hibbard, J. E., et al. 2005, ApJ, 619, L87

Hill, J. K., et al. 1992, ApJ, 395, L37

Hinz, J. L., Misselt, K., Rieke, M. J., Rieke, G. H., Smith, P. S., Blaylock, M., \& Gordon, K. D. 2006, ApJ, 651, 874

Hodge, P. W., \& Kennicutt, R. C., Jr. 1983, AJ, 88, 296

Hogg, D., \& Roberts, M. 2001, in ASP Conf. Ser. 240, Gas and Galaxy Evolution, ed. J. E. Hibbard, M. Rupen, \& J. H. van Gorkom (San Francisco: ASP), 859

Hoopes, C. G., et al. 2005, ApJ, 619, L99

Hunter, D. A., \& Elmegreen, B. G. 2006, ApJS, 162, 49

Ibata, R., Chapman, S., Ferguson, A. M. N., Lewis, G., Irwin, M., \& Tanvir, N. 2005, ApJ, 634, 287

Icke, V. 1985, A\&A, 144, 115

Iglesias-Paramo, J., et al. 2006, ApJS, 164, 38

Iyer, M., Simpson, C. E., Gottesman, S. T., Harsgis, J. R., \& Malphrus, B. K. 2001, in ASP Conf. Ser. 240, Gas and Galaxy Evolution, ed. J. E. Hibbard, M. Rupen, \& J. H. van Gorkom (San Francisco: ASP), 861

Jarrett, T. H., Chester, T., Cutri, R., Schneider, S. E., \& Huchra, J. P. 2003, AJ, 125,525

Kamphuis, J., \& Briggs, F. 1992, A\&A, 253, 335

Karachentsev, I. D. 1994, Astron. Astrophys. Trans., 6, 1

Karachentsev, I. D., et al. 2000, A\&A, 363, 117

. 2003, A\&A, 404, 93

Karachentseva, V. E. 1973, Astrofiz. Issledovanija Byu. Spec. Ast. Obs., 8, 3

Karachentseva, V. E., \& Karachentsev, I. D. 1998, A\&AS, 127, 409

Kazarian, M. A. 1996, Astrophysics, 39, 245

Kenney, J. D. P., Rubin, V. C., Planesas, P., \& Young, J. S. 1995, ApJ, 438, 135 Kennicutt, R. C., Jr. 1989, ApJ, 344, 685

. 1998, ARA\&A, 36, 189

Kennicutt, R. C., Jr., et al. 2003, PASP, 115, 928

Kereš, D., Katz, N., Weinberg, D. H., \& Davé, R. 2005, MNRAS, 363, 2 2006, ApJS, 162, 97

Koribalski, B. S., et al. 2004, AJ, 128, 16

Kornreich, D. A., Haynes, M. P., Lovelace, R. V. E., \& van Zee, L. 2000, AJ, 120, 139 
Lelièvre, M., \& Roy, J.-R. 2000, AJ, 120, 1306

Lovelace, R. V. E., Zhang, L., Kornreich, D. A., \& Haynes, M. P. 1999, ApJ, 524, 634

MacArthur, L. A. 2006, Ph.D. thesis, Univ. of British Columbia

MacArthur, L. A., Courteau, S., Bell, E., \& Holtzman, J. A. 2004, ApJS, 152, 175

Makarova, L. N., et al. 2002, A\&A, 396, 473

Maloney, P. 1993, ApJ, 414, 41

Marcum, P. M., et al. 2001, ApJS, 132, 129

Martin, C. L., \& Kennicutt, R. C., Jr. 2001, ApJ, 555, 301

Martin, D. C., et al. 2005, ApJ, 619, L1

Meurer, G. R., et al. 2006, ApJS, 165, 307

Minchin, R., et al. 2005, ApJ, 622, L21

Mo, H. J., Mao, S., \& White, S. D. M. 1998, MNRAS, 295, 319

Moore, E. M., \& Gottesman, S. T. 1998, MNRAS, 294, 353

Morrissey, P., et al. 2007, ApJS, 173, 682

Muñoz-Mateos, J. C., Gil de Paz, A., Boissier, S., Zamorano, J., Jarrett, T., Gallego, J., \& Madore, B. F. 2007, ApJ, 658, 1006

Noordermeer, E., van der Hulst, J. M., Sancisi, R., Swaters, R. A., \& van Albada, T. S. 2005, A\&A, 442, 137

Oosterloo, T., \& van Gorkom, J. 2005, A\&A, 437, L19

Pellerin, A., Meyer, M., Harris, J., \& Calzetti, D. 2007, ApJ, 658, L87

Pisano, D. J., \& Wilcots, E. M. 1999, AJ, 117, 2168 2003, ApJ, 584, 228

Pisano, D. J., Wilcots, E. M., \& Liu, C. T. 2002, ApJS, 142, 161

Pohlen, M., Dettmar, R.-J., Lütticke, R., \& Aronica, G. 2002, A\&A, 392, 807

Pohlen, M., \& Trujillo, I. 2006, A\&A, 454, 759

Pohlen, M., Beckman, J. E., Hüttemeister, S., Knapen, J. H., Erwin, P., \& Dettmar, R.-J. 2004, in Penetrating Bars Through Masks of Cosmic Dust, ed. D. L. Block et al. (Dordrecht: Kluwer), 713

Puche, D., Carignan, C., \& Bosma, A. 1990, AJ, 100, 1468

Rao, S., \& Briggs, F. 1993, ApJ, 419, 515

Rogstad, D. H., \& Shostak, G. S. 1971, A\&A, 13, 99

Rownd, B. K., Dickey, J. M., \& Helou, G. 1994, AJ, 108, 1638

Ryan-Weber, E. V., et al. 2004, AJ, 127, 1431

Salo, H. 1991, A\&A, 243, 118

Sandage, A., \& Bedke, J. 1994, The Carnegie Atlas of Galaxies (Washington: Carnegie Inst.)

Sandage, A., \& Brucato, R. 1979, AJ, 84, 472

Schaye, J. 2004, ApJ, 609, 667

Sicking, F. J. 1997, Ph.D. thesis, Univ. of Groningen

Smith, B. J. 1991, ApJ, 378, 39 1997, AJ, 114, 2177

Stewart, S. G., \& Walter, F. 2000, AJ, 120, 1794

Sun, W.-H., et al. 2005, ApJ, 630, L133

Swaters, R. A., van Albada, T. S., van der Hulst, J. M., \& Sancisi, R. 2002, A\&A, 390,829

Taga, M., \& Iye, M. 1998, MNRAS, 299, 1132
Thilker, D. A., Walterbos, R. A. M., Braun, R., \& Hoopes, C. G. 2002, AJ, 124, 3118

Thilker, D. A., et al. 2005a, ApJ, 619, L79

2005b, BAAS, 37, 1500

2007, ApJS, 173, 572

Thornley, M. D., \& Mundy, L. G. 1997, ApJ, 490, 682

Trujillo, I., \& Pohlen, M. 2005, ApJ, 630, L17

van der Hulst, J. M. 2002, in ASP Conf. Ser. 276, Seeing Through the Dust: The Detection of $\mathrm{H}$ I and the Exploration of the ISM in Galaxies, ed. A. R. Taylor,

T. L. Landecker, \& A. G. Willis (San Francisco: ASP), 84

van der Hulst, J. M., \& Sancisi, R. 2005, in ASP Conf. Ser. 331, Extra-Planar Gas, ed. R. Braun (San Francisco: ASP), 139

van der Hulst, J. M., van Albada, T. S., \& Sancisi, R. 2001, in ASP Conf. Ser. 240, Gas and Galaxy Evolution, ed. J. E. Hibbard, M. Rupen, \& J. H. van Gorkom (San Francisco: ASP), 451

van der Hulst, T., \& Sancisi, R. 1988, AJ, 95, 1354

van der Kruit, P. C. 1979, A\&AS, 38, 15

van der Kruit, P. C., \& Searle, L. 1981, A\&A, 95, 105

van Driel, W., Kraan-Korteweg, R. C., Binggeli, B., \& Huchtmeier, W. K. 1998, A\&AS, 127, 397

van Gorkom, J. 1991, in ASP Conf. Ser. 16, Atoms, Ions and Molecules: New Results in Spectral Line Astrophysics (San Francisco: ASP), 1

van Gorkom, J. H., Knapp, G. R., Raimond, E., Faber, S. M., \& Gallagher, J. S. 1986, AJ, 91, 791

van Zee, L. 2001, AJ, 121, 2003

Varela, J., Moles, M., Márquez, I., Galletta, G., Masegosa, J., \& Bettoni, D. 2004, A\&A, 420, 873

Verdes-Montenegro, L., Bosma, A., \& Athanassoula, E. 2000, A\&A, 356, 827

Verdes-Montenegro, L., Sulentic, J., Lisenfeld, U., Leon, S., Espada, D., Garcia, E., Sabater, J., \& Verley, S. 2005, A\&A, 436, 443

Vollmer, B., Braine, J., Combes, F., \& Sofue, Y. 2005, A\&A, 441, 473

Vollmer, B., Soida, M., Otmianowska-Mazur, K., Kenney, J. D. P., van Gorkom, J. H., \& Beck, R. 2006, A\&A, 453, 883

Waller, W. H., et al. 1997, ApJ, 481, 169

Walter, F. 2001, Ap\&SS Suppl., 277, 91

Walter, F., \& Brinks, E. 1999, AJ, 118, 273

Werk, J. K., Putman, M. E., Meurer, G. R., Oey, M. S., \& Ryan-Weber, E. V. 2007, in IAU Symp. 237, Triggered Star Formation in a Turbulent ISM, ed.

B. G. Elmegreen \& J. Palous (Cambridge: Cambridge Univ. Press), 493

White, S. D. M., \& Frenk, C. S. 1991, ApJ, 379, 52

Yun, M. S., Ho, P. T. P., \& Lo, K. Y. 1994, Nature, 372, 530

2000 , in ASP Conf. Ser. 217, Imaging at Radio through Submillimeter Wavelengths, ed. J. G. Mangum \& S. J. E. Radford (San Francisco: ASP), 374

Zaritsky, D., \& Christlein, D. 2007, AJ, 134, 135

Zaritsky, D., Kennicutt, R. C., Jr., \& Huchra, J. P. 1994, ApJ, 420, 87

Zaritsky, D., Smith, R., Frenk, C., \& White, S. D. M. 1997, ApJ, 478, 39 